\title{
The Sun's Movement in the Sky Now and in the Past
}

\author{
Joseph J. Smulsky \\ Institute of the Earth's Cryosphere, Tyumen Scientific Centre, Siberian Branch of Russian Academy of Sciences, Tyumen, Russia \\ Email: jsmulsky@mail.ru
}

How to cite this paper: Smulsky, J.J. (2018) The Sun's Movement in the Sky Now and in the Past. Open Access Library Journal, 5: e4250.

https://doi.org/10.4236/oalib.1104250

Received: December 12, 2018

Accepted: April 5, 2018

Published: April 8, 2018

Copyright (c) 2018 by author and Open Access Library Inc.

This work is licensed under the Creative Commons Attribution International License (CC BY 4.0).

http://creativecommons.org/licenses/by/4.0/

\begin{abstract}
The paper considers the theory of the phenomena associated with the Sun's passage in the sky when the parameters of the orbital and rotational motion of the Earth are changing. The author found that the obliquity changes from $14.8^{\circ}$ to $32.1^{\circ}$. These results explain the variations of paleoclimate and are consistent with several testimonies of paleoastronomy. The program SunPhenomn.mcd developed in MathCad to calculate the duration of solar day, equation of time, daylight, azimuths of the daily movement of the Sun, sunrises and sunsets, the length and azimuth of the gnomon shadow, duration of the polar days and nights, of seasons and other phenomena. The phenomena of the Sun in the contemporary epoch and in 4 extreme epochs in the interval of 50 thousand years ago are calculated. The example of the funnel on Yamal shows the application of the program SunPhenomn.mcd in aerospace studies of the relief. The work is of interest to a wide range of experts in the field of Earth Sciences and can be used by students and post-graduate students to carry out theses and dissertations.
\end{abstract}

\section{Subject Areas}

Functional Analysis, Mathematical Analysis

\section{Keywords}

Changing Parameters, Orbit, The Earth's Axis, Duration, Day, Polar Night, Gnomon

\section{1. Введение}

Со временем мы все больше узнаем о прошлом человечества. Не так давно, найденные в Диринг-Юряхе, в Якутии, архаичные орудия древнего человека, по мнению археолога Ю. А. Мочанова, имеют возраст порядка 
2-x - 3-х миллионов лет [1]. С другой стороны, на основании сравнения ДНК древних останков человека и современного местного населения разных континентов другие ученые пришли к выводу о том, что современный тип людей вышел из Африки несколько десятков тысяч лет назад [2]. Эти противоречивые выводы требуют дальнейшего изучения прошлого человечества.

Наиболее достоверным свидетельством о древнем человеке являются следы его деятельности, в том числе и древние календари. Использование календарей свидетельствует о высоком уровне развития древнего общества. Чтобы создать календарь должна быть не менее чем тысячелетняя база осознанных наблюдений явлений природы. Например, Геродот (484 - 425 гг. до н. э.) пишет, что увиденные им захоронения 330 верховных жрецов свидетельствуют об истории Египта на протяжении десяти тысячелетий. На основании наблюдений древних жрецов был создан Египетский календарь, в котором год состоял из 12 месяцев по 30 дней и пяти дополнительных дней.

Календари основываются на повторяющихся явлениях природы, самые главные из которых связаны с ежедневными появлениями и исчезновениями Солнца. От движения Солнца по небосводу зависят длительность суток, долгота светового дня, время наступления восходов и заходов Солнца и их азимуты, высота Солнца над горизонтом в полдень, длительность сезонов и моменты их начала, длительность полярных дней и ночей в высоких широтах и их начала и др. Все эти феномены Солнца определяли жизнь древнего человека. Поэтому он создавал разнообразные календари, которые позволяли планировать его деятельность.

Древние календари и даже древние обсерватории, могли существенно отличаться друг от друга. Одни из них были монументальные, как Стоун Хендж в Англии, а другие-в виде амулетов или женских украшений. Интерпретация древних календарей может быть разнообразной. Даже те, которые всемирно известны и хорошо изучены, интерпретируются по-разному.

Для этого есть причины. Современному человеку бывает достаточно трудно понять потребности древнего человека и, соответственно, понять назначение элементов древнего календаря. Во-вторых, календари зависят от местности, в которой проживал древний народ, от его уклада: кочевой он был или оседлый, а также от многих других обстоятельств, которые полностью можно понять нам самим, превратившись в древний народ. В-третьих, параметры орбиты Земли и оси ее вращения со временем изменяются. Поэтому древние предназначения элементов календаря в современную эпоху уже не выполняют своей роли.

В этой работе представлена теория феноменов Солнца. На мой взгляд, она позволит адекватно интерпретировать разнообразные древние календари. В современной астрономии, по-видимому, имеются теории практически всех явлений Солнца. Они основаны на уравнении Кеплера, которым определяется время движения Земли по орбите (или Солнца на небесной 
сфере) в зависимости от углового положения Земли. Переменные этого уравнения: эксцентрическая и истинная аномалия, корнями восходят к древней астрономии, и, на мой взгляд, как физически, так и математически представляют некую таинственность, которая затрудняет понимание этих теорий.

Я рассматриваю точные результаты решения задачи двух тел: Земли и Солнца в полярной системе координат $r(\varphi)$. В зависимости от радиуса $r$ или угла $\varphi$ получены время $t$ движения по орбите при разные ее видах: эллипс, парабола, гипербола или радиальная прямая [3]. Поэтому всю теорию я вывожу по-новому, несмотря на то, что теория практически всех явлений, как я уже упоминал, по-видимому, есть. Все результаты новой теории сопоставлены с результатами традиционной. Поэтому у меня нет сомнения, что где-то могла закрасться ошибка.

Создание этой теории не являлось самоцелью. Главное ее назначение рассчитывать феномены Солнца в другие эпохи, когда параметры орбиты Земли и оси ее вращения были другие. Поэтому необходимо было бы весь вывод прежней теории проверять: остается ли она справедливой при изменении этих параметров. Как выше отмечено, был выбран путь создания новой теории. На мой взгляд, она значительно проще и понятнее. Лучше поддается алгоритмизации. Поэтому эта работа и созданная программа SunPhenomn.mcd расчета феноменов Солнца позволит исследователям, не имеющим фундаментального образования по астрономии, исследовать и интерпретировать древние календари.

Созданная М. Миланковичем Астрономическая теория изменения палеоклимата почти 100 лет назад основывается на решении трех проблем: 1) как изменяется орбита Земли; 2) как изменяется ось вращения Земли; 3) как изменяется освещенность (или инсоляция) Земли Солнцем в зависимости от параметров орбитального и вращательного движений Земли. Все эти три задачи я решил по-новому и по-другому [3] [4] [5]. По второй задаче получены существенные отличия от прежней теории. Они заключаются в том, что ось Земли имеет большие колебания: от $32^{\circ}$ до $15^{\circ}$ при современном наклоне оси Земли $23.4^{\circ}$ к перпендикуляру плоскости ее орбиты. По прежним теориям этот наклон изменяется от $22.26^{\circ}$ до $24.32^{\circ}$. Таким образом, колебания оси Земли в новой теории в 7 - 8 раз больше по сравнению с прежними колебаниями. Во столько раз больше колебания инсоляции. Кроме того, получены другие периоды колебаний и другие моменты наступления экстремумов этих колебаний.

При сопоставлении новых результатов Астрономической теории с палеоклиматом, например, в Западной Сибири, выяснилось, что экстремумы палеоклимата совпадают с экстремумами инсоляции: потепление в Голоцене 4.2 тысяч лет назад (т.л.н.), Сартинское оледенение 15.9 т.л.н., Каргинское межледнивековье 31.3 т.л.н. и Ермаковский ледниковый период 46.4 т.л.н. [3].

В ледниковые периоды на шельфе и побережье Карского моря образовался 
Ледниковый щит, который соприкасался со Скандинавским щитом. Высота ледниковых щитов достигала 1.5 - 2 км. Перекрывался сток рек Оби и Енисея в Ледовитый океан, и Западно-Сибирская равнина превращалась в пресноводное море. Уровень его повышался и через Тоболо-Тургайскую ложбину шел сток в Приаралье. Затем по южному проходу вода из Западно-Сибирского моря попадала в Каспий. При повышении уровня Каспия больше +27 м вода сибирских рек по Кумо-Манычской впадине могла проходить в Черное море.

Сток льда с Карского ледникового щита создавал ряд морен, самая южная гряда которых образовала Сибирские Увалы. Параллельными рядами остатки древних морен простираются до самого Карского моря.

В эти же эпохи происходили аналогичные события в Европе [6] и в Северной Америке [7]. Ледниковые периоды приводили к кардинальным изменениям в природе, в том числе и в судьбе человечества. В вышеупомянутых генетических исследованиях [2] миграция человека из Африки вдоль побережья Индийского океана в Полинезию и Австралию, затем вдоль берегов Тихого океана в Китай, Японию, Чукотку и через современный Берингов пролив в Америку приурочивается к ледниковым эпохам. Как уже упоминалось, можно усомниться в одноцентровом очаге зарождения человечества, но в ледниковых периодах, как причинах миграции человека, многие авторы единодушны.

Календари древних народов, как те, которые найдены, так и те, которые откроют в будущем, отражают параметры орбитального и вращательного движения Земли. Последние привязаны ко времени с высокой точностью, например, с погрешностью 6 сек за 200 тыс. лет. Поэтому с подобной точностью могут быть датированы эпохи древних календарей.

В древних календарях также большую роль играют феномены Луны. В данной работе они не рассматриваются. Однако база для них имеется. Эволюцию орбитального движения планет и Луны я исследовал за 100 млн. лет [8]. Выяснилось, что ось орбиты Луны вращается (прецессирует) вокруг оси подвижной орбиты Земли с периодом 18.6 лет. Вращение происходит с небольшими колебаниями. На небесной сфере это отражается в том, что плоскость орбиты Луны колеблется в диапазоне $\pm 5^{\circ}$ по отношению к эклиптике - пути Солнца на небесной сфере. И эти колебания неизменны в течение 100 млн. лет. Поэтому на приведенный алгоритм в программе SunPhenomn.mcd нужно наложить алгоритм изменения современных феноменов Луны, и он автоматически будет подстраиваться под изменяющиеся параметры орбиты Земли. Надеюсь, в дальнейшем такое дополнение будет сделано.

Рассматриваемая теория явлений Солнца, в том числе и солнечной тени, и программа их расчета могут быть использованы и для других целей, например при аэрокосмических исследованиях рельефа Земли и планет. В работе, как пример использования, рассматривается профиль бугра, 
который предшествовал появлению воронки на Ямале осенью 2013 г. Форма профиля может свидетельствовать о возможном механизме образования воронки.

В завершение отметим, что разработанная теория позволит повысить степень определенности в интерпретации древних календарей, и тем самым наши знания о прошлом человечества будут иметь большую достоверность.

Как читать эту работу? При первом чтении нужно отвлечься от формул и их выводов и познакомиться с общим содержанием работы: о чем идет речь? При втором чтении нужно вникнуть в содержание рисунков и графиков: что на них изображено? При третьем чтении уже можно познакомиться с выводом формул и обоснованием программы на SunPhnmen.mcd. А тем, кто решил ее использовать, нужно познакомиться с содержанием программы и произвести расчеты разных феноменов Солнца для широты места своего нахождения в современную эпоху $T=0$ и в любую другую эпоху $T \neq 0$.

\section{2. Новые решения по эволюции вращательного движения Земли}

Новые решения задачи об эволюции вращательного движения Земли [9] [10] показаны линией 1 на Рис. 1 в виде эволюции угла наклона $\varepsilon$ за прошедшие 200 тыс. лет. Угол $\varepsilon$-это угол между плоскостями экватора Земли и ее орбиты. Он изменяется от $14.8^{\circ}$ до $32.1^{\circ}$ [3] при современном его значении $23.4^{\circ}$. Примерно такой же диапазон изменения $\varepsilon$ получен при решении задачи за будущие 200 тыс. лет [11]. В то же время по прежним теориям, например [12] (см. Рис. 1, линия 2) угол $\varepsilon$ изменялся от $22.21^{\circ}$ до $24.43^{\circ}$. Из приведенных значений видно, что диапазон колебания угла наклона по новым решениям в 7 - 8 раз больше, чем по прежним теориям.

Эти результаты на протяжении нескольких лет проверялись. Задача была решена еще тремя другими методами [3] и [13]. Результаты неизменились. Кроме того, полученные в этой задаче изменения параметров вращательного

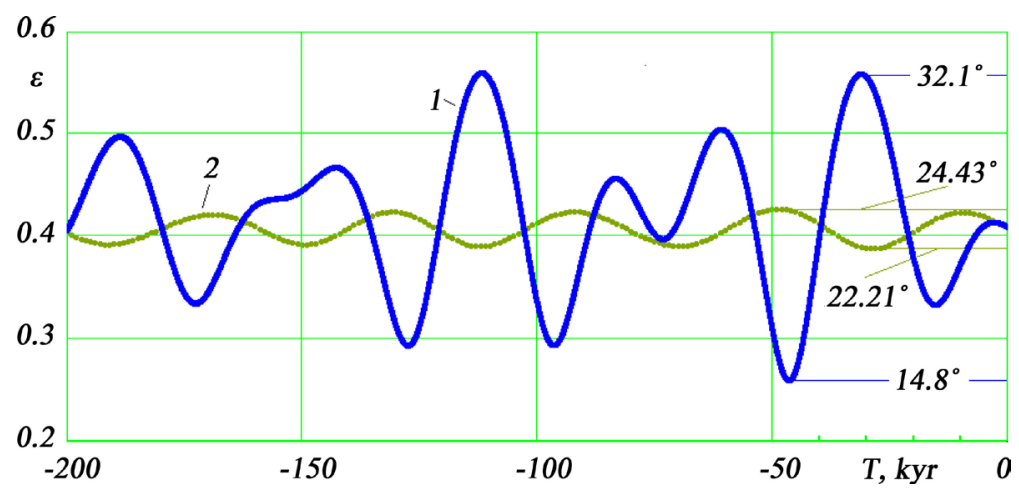

Рис. 1. Эволюция угла наклона $\varepsilon$ (в радианах) экватора Земли к плоскости ее орбиты за 200 тыс. лет в прошлое. Сравнение новых результатов 1 с результатами прежних теорий 2 на примере работы Дж. Ляскара и др. [12]. В градусах приведены максимальные и минимальные значения угла $\mathcal{\varepsilon}$. $T$-время в тыс. лет от 30.12 .1949 г. 
движения Земли на интервале нескольких тысяч лет, для которых имеются наблюдения, совпали с данными наблюдений.

Как видно из Рис. 1, новые решения 1 отличаются от прежних 2 не только амплитудами колебания, но и моментами наступления экстремумов. Эти отличия в эволюции угла наклона $\varepsilon$ приводят к таким же существенным отличиям в эволюции инсоляции Земли [3] и [11]. При сопоставлении нового изменения инсоляции за прошедшие 50 тыс. лет с палеоклиматом было установлено [4] и [8], что оно объясняет все известные его колебания: суровый ледниковый период 46 тыс. лет назад, сильное потепления 31 тыс. лет назад, последний ледниковый период 16 тыс. лет назад и небольшое потепления 4 тыс. лет назад.

Существуют свидетельства, что угол наклона $\varepsilon$ изменялся более существенно, чем это следует из прежних теорий. Например, М. И. Исрапилов [14] исследовал сотни древних календарей в Дагестане. Он пришел к выводу, что угол наклона $\varepsilon$ изменялся от $19^{\circ}-20^{\circ}$ до $32^{\circ}-33^{\circ}$. Возраст древних календарей у него доходит до 65 тыс. лет. Б. Г. Тилак [15] исследовал тексты Вед, Бхагавад-Гиты, Авесты и др. древних источников. В них используются характеристики полярной области, как будто древние арии обитали в ней. Это можно объяснить только большими углами наклона $\varepsilon$. В этом случае полярный круг проходит южнее современного и, дополнительно, на этих широтах становится теплее. Тем самым создаются необходимые для жизни человека условия в полярной области.

Ряд календарей в Дагестане М. И. Исрапилов [14] называет обсерваториями, т.к. они позволяли определять моменты наступления лунных и солнечных затмений. Таких обсерваторий найдено достаточно много, в том числе Стоунхендж в Англии [16] и Ales Stones на юге Швеции [17]. Многие из них, в том числе последние ориентированы по азимуту восхода Солнца в дни летнего и зимнего солнцестояния. Такие обсерватории и календари называют азимутальными. В зенитных обсерваториях и календарях [14] объекты ориентированы так, чтобы можно было фиксировать зенитные углы солнцестояний, равноденствий и других моментов, важных для хозяйственной деятельности человека. В календарях третьего вида, которые были распространены на Алтае и в Сибири [18], календари выполнялись в виде жезлов, пластин, фигур животных и даже женских украшений. На них насечками или другим способом отмечены дни года и моменты наступления различных феноменов Луны и Солнца. По этим календарям можно определить продолжительность интервалов между днями равноденствий и солнцестояний, а также временные характеристики других астрономических явлений.

Характеристики календарей зависят от параметров орбитального и вращательного движения Земли, а также от широты места нахождения календаря. Однако зависимость эта сложна и не очевидна. Для понимания механизма функционирования календаря и его расшифровки необходимо 
использовать теорию солнечных явлений. Имеющееся в астрономии описание этих феноменов достаточно сложное. Оно не позволяет исследователям древних календарей оперативно рассчитывать условия применительно к рассматриваемому календарю и подбирать различные варианты параметров, от которых зависят зафиксированные календарем явления.

Целью настоящей работы является создание такой теории. В отличии от существующей таковой в астрономии, в ней, как уже отмечалось, используется другой алгоритм определения долгот Солнца при его годовом движении. Кроме того, все математические операции алгоритмизированы и представлены в виде программы на SunPhnmen.mcd в среде MathCad. Для ориентировки исследователей древних календарей рассчитаны солнечные феномены при современных и при отличающихся от современных параметрах орбитального и вращательного движения Земли. Они взяты для четырех экстремумов $\varepsilon$ за последние 50 тыс. лет (см. Рис. 1): $T=2.8 ; 15.32 ; 31$ и 46.44 тыс. лет назад.

\section{3. Геометрические характеристики движения Солнца по небосводу}

\section{1. Движение Солнца в течение года}

Рассмотрим положение Солнца $S$ над точкой $M$ земной поверхности (Рис. 2). Плоскость горизонта в т. $M$ на небесной сфере 1 нанесена горизонтальным кругом $H^{\prime}$. Перпендикуляр к плоскости $H^{\prime}$ пересекает поверхность небесной сферы 1 в точке зенита $Z$. Солнце $S$ совершает вокруг Земли годовое движение по орбите, которая проектируется на небесную сферу в виде круга эклиптики $E E$. Движение происходит против стрелки часов с началом отсчета долготы $\lambda$ Солнца в точке весеннего равноденствия $\gamma$. этой точке Солнце находится в плоскости экватора $A A^{\prime}$, когда из южного полушария переходит в северное.

Видимое орбитальное движение Солнца вокруг Земли обусловлено неравномерным движением Земли вокруг Солнца по орбите, которая является эллипсом. Относительно неподвижных звезд период движения по орбите $P_{s d}=365.25636042$ дней. Он называется сидерическим годом. В работах [19] [20] представлен алгоритм расчета долгот Солнца $\lambda_{j 1}$, где $j 1=1,2, \cdots, 365$, по дням года $T_{d, j 1}=1,2, \cdots, 365$. Отсчет дней начинается от момента весеннего равноденствия, т.е. $j 1=0$ соответствует моменту равноденствия. Этот алгоритм основан на точном решении задачи двух тел [21] [22]. Задача рассматривается в полярной системе координат $\left(r, \varphi_{o}\right)$, где $r$ - расстояние Земли от Солнца, а $\varphi_{o}$ - угловое положение Земли на орбите относительно перигелия орбиты. Время $t_{f p}$ движения Земли по орбите от точки перигелия до точки ее нахождения с углом $\varphi_{о}$ рассчитывается по формуле

$$
t_{f p}=t_{f p}^{\prime} \text { при } \varphi_{o} \leq \pi \text { и } t_{f p}=2 \cdot t_{a}-t_{f p}^{\prime} \text { при } \pi<\varphi_{o} \leq 2 \times \pi,
$$




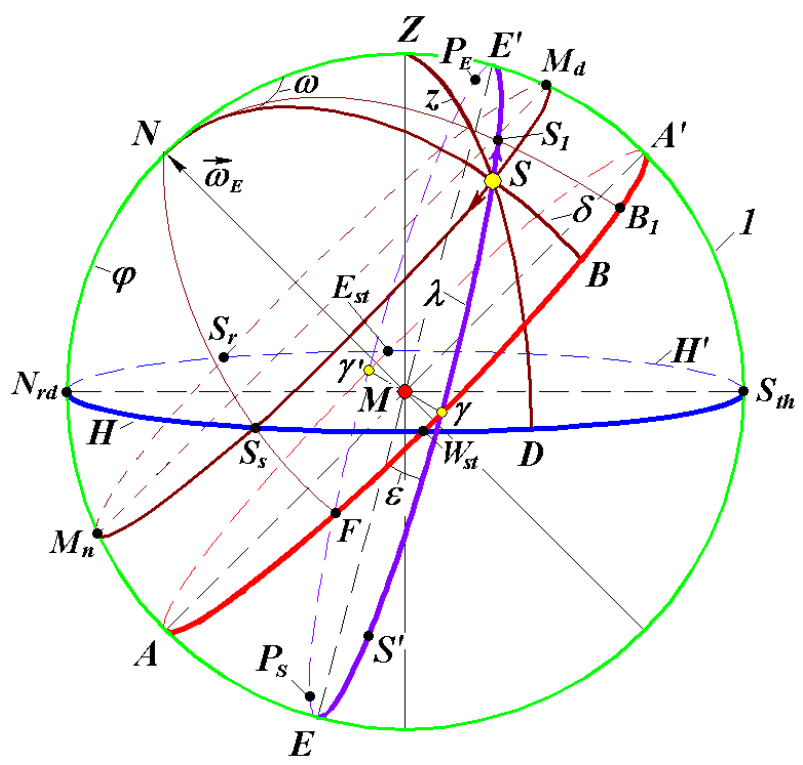

Рис. 2. Основные геометрические характеристики Солнца $S$ и наблюдателя $M$ на земной поверхности. Характеристики небесной сферы 1: $A A^{\prime}$-плоскость подвижного экватора; $E E$-плоскость подвижной эклиптики, а $\varepsilon$-угол между плоскостями $A A^{\prime}$ и $E E ; N$-северный полюс; Характеристики плоскости горизонта $H H^{\prime}$ : дуга $N_{r d} N=$ $\varphi$ - географическая широта точки $M$ на плоскости горизонта; $Z$ - зенит точки $M$; $N_{r \phi} E_{s p} S_{t h}$ и $W_{s t}$ - точки Севера, Востока, Юга и Запада, соответственно, на круге горизонта $H H^{\prime}$. Характеристики годового движения Солнца по кругу эклиптики $E E$ : $\delta=S B$ - склонение Солнца; $\lambda=\gamma S$ - долгота Солнца; точки $\gamma, E, \gamma$ и $E$-точки нахождения Солнца в дни весеннего равноденствия, летнего солнцестояния, осеннего равноденствия и зимнего солнцестояния, соответственно; $S S_{1}$ - путь Солнца за одни солнечные сутки. Характеристики суточного движения Солнца по кругу $S_{r} M_{d} S_{s} M_{n}$ : точки $S_{t}, M_{d}, S_{s}$ и $M_{n}$ положения центра Солнца в моменты восхода, полудня, захода и полуночи, соответственно; $z=\angle Z M S$ - зенитный угол Солнца; $\omega$ $=\angle Z N S-$ часовой угол Солнца, отсчитываемый от полудня.

где

$t_{f p}^{\prime}=\frac{R_{p}}{v_{p}} \cdot\left\{\frac{\left(\alpha_{1}+1\right)\left|\sin \varphi_{o}\right|}{\left(2 \alpha_{1}+1\right)\left[\left(\alpha_{1}+1\right) \cos \varphi_{o}-\alpha_{1}\right]}+\frac{\alpha_{1}\left[\arcsin \frac{\alpha_{1}+1-\alpha_{1} \cos \varphi_{o}}{\left(\alpha_{1}+1\right) \cos \varphi_{o}-\alpha_{1}}-\pi / 2\right]}{\left(-2 \alpha_{1}-1\right)^{3 / 2}}\right\}$

$R_{p}$ - радиус перигелия;

$v_{p}$-скорость Земли в перигелии.

$\alpha_{1}=\mu_{1} /\left(R_{p} v_{p}^{2}\right)$-параметр траектории;

$\mu_{1}=-G(M+m)$-параметр взаимодействия;

$G$-гравитационная постоянная;

$M$-масса Солнца;

$m$-масса Земли;

$t_{a}=\frac{R_{p}}{v_{p}} \cdot \frac{-\pi \alpha_{1}}{\left(-2 \alpha_{1}-1\right)^{3 / 2}}$-время движения от перигелия до афелия, которое 
следует из (2) при $\varphi_{o}=\pi$. Здесь используется параметр траектории $\alpha_{1}$, так как он известен при постановке задачи, а эксцентриситет орбиты $e$ определяется в результате решения задачи двух тел. Для гравитационного взаимодействия эти параметры однозначно связаны: $e=-\left(1+\alpha_{1}\right) / \alpha_{1}$.

Этот алгоритм отличается от традиционного, основанного на уравнении Кеплера. Он, как уже отмечалось, базируется на точном решении задачи двух тел и является более простым в использовании. Программа Insl2bd.mcd в среде MathCad для расчета долгот $\lambda_{j 1}$ и других характеристик инсоляции Земли имеется в свободном доступе:

http://www.ikz.ru/ smulski//Data/Insol/. Алгоритм для определения долгот $\lambda_{j 1}$ включен в пункты 3 - 5 программы на SunPhnmen.mcd расчета феноменов Солнца, которая представлена в Приложении. Текст программы на SunPhnmen.mcd также имеется в свободном доступе: http://www.ikz.ru/ smulski//Data/Insol/.

Как уже отмечалось, формулами (1)-(2) определяется время движения Земли от точки положения ее перигелия $P_{E}$ (см. Рис. 2). Перигелий относительно восходящего узла $\gamma$ определяется углом $\varphi_{p \gamma}$ Относительно Земли Солнце движется по той же орбите, но его перигей $P_{S}$ сдвинут на $180^{\circ}$, т.е. угол перигея Солнца $v=\varphi_{p \gamma}+\pi$. Так как долгота Солнца $\lambda$, также отсчитывается от восходящего узла $\gamma$ (см. Рис. 2), то полярный угол $\varphi_{о}$ в формулах (1)-(2) выразится через долготу $\lambda$ так:

$$
\varphi_{o}=\lambda-v=\lambda-\varphi_{p \gamma}-\pi
$$

Итак, формулами (1)-(3) определяется время годового движения Солнца по небесной сфере, т.е. по эклиптике $E E^{\prime}$, в зависимости от его долготы $\lambda$.

Эксцентриситет орбиты $e$, угол перигелия $\varphi_{p \gamma}$ и угол наклона орбиты $\varepsilon$ к экватору изменяются со временем. Они определяются в результате решения двух задач об орбитальном и вращательном движении Земли. В рассматриваемых программах в среде MathCad эти параметры в зависимости от времени $T$, считываются из файла, например, файла OrAl-200.prn - за 200 тысяч лет назад (т.л.н.).

Положение Солнца $S$ (Рис. 2) на эклиптике $E E^{\prime}$ известно в любой момент времени $t$. А угловое расстояние Солнца $S B$ от экватора, т.е. угол склонения $\delta$, определяется известным в астрономии выражением

$$
\delta=\arcsin (\sin \varepsilon \cdot \sin \lambda) .
$$

Вывод выражения (4) имеется в работах [19] [20]. Отметим, что все используемые в настоящей работе формулы выведены нами. Многие из них известны в астрономии. Однако необходимо осуществлять их вывод по-новому, чтобы быть уверенным в возможности их использования при измененных параметрах орбитального и вращательного движения Земли.

Если в формулу (4) подставить $\lambda=\lambda_{j 1}$, то мы получим склонение $\delta_{j 1}$ для каждого дня года. В этой формуле и в последующих, с целью упрощения изложения, индекс $j 1$ опускаем. Координатами $\lambda$ и $\delta$ полностью определяется 
годовое движение Солнца по небосводу. В дальнейшем потребуется угловое расстояние Солнца $\gamma B$ (Рис. 2) на экваторе $A A^{\prime}$, которое известно в астрономии как прямое восхождение $\alpha_{s}$. Здесь индекс $S$, обозначающий Солнце, введен для отличия при обозначении значком $\alpha$ других параметров.

В сферическом прямоугольном треугольнике $\gamma S B$ известны углы: $B=\pi / 2$; $\gamma=\mathcal{E}$ и две стороны: $\gamma S=\lambda, B S=\delta$. Согласно преобразованиям сферической геометрии, например, формулы (1.1.013) в работе [23] можем записать для неизвестной стороны $\gamma B=\alpha_{\dot{S}}$ :

$$
\sin \alpha_{S}=\operatorname{tg} \delta \cdot \operatorname{ctg} \varepsilon .
$$

После подстановки в это выражение $\delta$ из (4) получаем прямое восхождения Солнца в следующем виде:

$$
\alpha_{S 0}=\arcsin \frac{\cos \varepsilon \cdot \sin \lambda}{\sqrt{1-(\sin \varepsilon \cdot \sin \lambda)^{2}}} ;
$$

$\alpha_{S}=\alpha_{\text {S0 }}$ при $\lambda \leq 0.5 \pi ; \alpha_{S}=\pi-\alpha_{\text {S0 }}$ при $0.5 \pi<\lambda \leq 1.5 \pi ; \quad \alpha_{S}=2 \pi+\alpha_{\text {S0 }}$ при $\lambda>1.5 \pi$.

В формулу (5) входит долгота Солнца $\lambda$, которая изменяется от 0 до $2 \pi$. Так как функция $\arcsin$ многозначна, то на интервалах $\lambda$ больших $\pi / 2$ необходимо выбирать нужные значения по представленному алгоритму.

Плоскости экватора $A A^{\prime}$ и эклиптики $E E^{\prime}$ изменяются в пространстве (Рис. 2), вследствие чего точка весеннего равноденствия $\gamma$ по часовой стрелке перемещается по эклиптике за год на 50".25641. Как уже отмечалось, годовое движение Солнца проходит по кругу эклиптики $E E^{\prime}$ против часовой стрелки, что отражается изменением долготы $\lambda$, начиная от точки весеннего равноденствия $\gamma$. Поэтому время прохождения Солнцем двух последовательных точек весеннего равноденствия, т.е. тропический год $P_{t r}=365.24219879$ дней, меньше сидерического года $P_{s d .}$ Для того, чтобы сезоны года не смещались по датам, современный календарь основан на тропическом годе.

Солнце $S$ при годовом движении по эклиптике $E E^{\prime}$ (см. Рис. 2) в момент весеннего равноденствия находится в т. $\gamma(\lambda=0)$, а в день зимнего солнцестояния Солнце проходит т. $E^{\prime}(\lambda=\pi / 2)$, в момент осеннего равноденствия находится в т. $\gamma^{\prime}(\lambda=\pi)$, а в день зимнего солнцестояния проходит т. $E(\lambda=3 \pi / 2)$. В п. 8 программы на SunPhnmen.mcd вычислены моменты в днях прохождения точек лета $T_{d s m}$, осени $T_{d a u}$ и зимы $T_{d w r}$. Точку весеннего равноденствия, как уже отмечалось, Солнце проходит в момент $T_{d s p}=0$. Так как ряд долгот $\lambda_{j 1}$ расположен по целочисленному ряду дней $T_{d, j l}$, то эти расчеты проводятся интерполяцией по соседним значением долгот. Продолжительности сезонов: весны, лета, осени и зимы рассчитываются по разностям этих моментов, соответственно:

$\Delta T_{d s p}=T_{d s m}-T_{d s p} ; \Delta T_{d s m}=T_{d a u}-T_{d s m} ; \Delta T_{d a u}=T_{d w n}-T_{d a u} ; \Delta T_{d w n}=P_{t r}-T_{d w n}$.

Результаты расчетов моментов наступления сезонов и их длительностей, 
согласно (6), приведены в табл. 1 для пяти эпох. Как уже отмечалось, эпохи выбраны в моменты наступления экстремумов угла наклона $\varepsilon$. Как видно из табл. 1 величина $\varepsilon$ изменяется от 0.25841 до 0.55875 в радианах, или от $14.8^{\circ}$ до $32.1^{\circ}$ в градусах. В современную эпоху $(T=0)$ наибольшую длительность имеет лето $\left(\Delta T_{d s m}=93.654\right.$ дня), а наименьшую-зима $\left(\Delta T_{d w n}=\right.$ 88.981 дня). В эпоху $T=2.8$ т.л.н лето имеет еще большую длительность, а наименьшую-осень. В эпоху $T=15.32$ т.л.н лето имеет наименьшую длительность, а наибольшую-осень. Аналогичные изменения длительности сезонов происходят в остальные эпохи. Однако эти изменения не очень большие и не превышают 6 дней.

В Таблице 1 приведены длительности сезонов для северного полушария. Эти данные применимы и для южного полушария, если весну заменить осенью, а лето-зимой. Следует отметить, что приведенные в табл. 1 длительности сезонов для современной эпохи $(T=0)$ совпадают с известными в астрономии.

\section{2. Суточное движение Солнца}

Земля совместно с наблюдателем $M$ (см. Рис. 2), кругом горизонта $H H^{\prime}$ и меридианом $N Z E^{\prime} M_{d} A^{\prime}$ вращается вокруг оси вращения Земли $M N$, с угловой скоростью $\vec{\omega}_{E}$. Вращение происходит против часовой стрелки. Поэтому Солнце относительно Земли и, в частности, относительно круга горизонта $H H^{\prime}$ по часовой стрелке перемещается по кругу $S_{r} M_{d} S_{s}$ параллельно экватору $A A^{\prime}$. В точке $S_{r}$ оно восходит над горизонтом $H H^{\prime}$, в точке $M_{d}$ находится в полдень, а в точке $S_{s}$ заходит за горизонт, а в точке $M_{n}$ находится в полночь. Часовой угол $\omega$ Солнца $S$ отсчитывается от меридиана $N Z E^{\prime} M_{d} A^{\prime}$, проходящего через точку полдня $M_{d \cdot}$ Часовой угол $\omega$ равняется дуге $A^{\prime} B$ на круге экватора $A A^{\prime}$. Угловое расстояние Солнца от зенита $Z$ определяется дугой $Z S=Z$, где $Z$ называется зенитным углом.

В сферическом треугольнике $N Z S$ известны две стороны: $N Z=\pi / 2-\varphi$; $N S=\pi / 2-\delta$ и угол $N=\omega$ между ними. Тогда по теореме косинусов [23] $\cos Z S=\cos N Z \cdot \cos N S+\sin N Z \cdot \sin \varphi N S \cdot \cos N$ для зенитного угла получим выражение в следующем виде [19] [20]:

$$
\cos Z=\sin \delta \cdot \sin \varphi+\cos \delta \cdot \cos \varphi \cdot \cos \omega .
$$

Таблица 1. Количество дней до начала сезонов в Северном полушарии и их продолжительность в эпохи $T$ наступления экстремумов угла наклона $\varepsilon: T-$ время в тыс. лет от 30.12.1949 г.

\begin{tabular}{cccccccccc}
\hline № & $T$ & $\mathcal{E}$ & $T_{d s m}$ & $T_{d a u}$ & $T_{d w n}$ & $\Delta T_{d s p}$ & $\Delta T_{d s m}$ & $\Delta T_{d a u}$ & $\Delta T_{d w n}$ \\
\hline 1 & 0 & 0.40916 & 92.770 & 186.425 & 276.261 & 92.770 & 93.654 & 89.837 & 88.981 \\
2 & -2.8 & 0.41343 & 94.226 & 186.029 & 274.484 & 94.226 & 91.803 & 88.454 & 90.759 \\
3 & -15.32 & 0.33237 & 88.484 & 181.276 & 275.458 & 88.484 & 92.792 & 94.183 & 89.784 \\
4 & -31 & 0.55875 & 91.191 & 179.887 & 271.280 & 91.191 & 88.697 & 91.393 & 93.962 \\
5 & -46.44 & 0.25841 & 92.806 & 185.831 & 275.649 & 92.806 & 93.026 & 89.818 & 89.593 \\
\hline
\end{tabular}


Зенитный угол $Z$ отсчитывается от точки зенита $Z$ против часовой стрелки. В точке восхода $S_{r}$ он равен: $Z=-\pi / 2$, в точке захода $S_{s}$ угол $Z=\pi / 2$. При этих углах из выражения (7) определяется часовой угол заходов и восходов Солнца.

$$
\omega_{0}= \pm \operatorname{arcos}(-\operatorname{tg} \varphi \cdot \operatorname{tg} \delta)
$$

В представленном на Рис. 2 положении наблюдателя $M$ и Солнца $S$ длительность дня больше длительности ночи. При нахождении Солнца $S$ в полдень в точке $E^{\prime}$ длительность дня будет наибольшая. Это точка летнего солнцестояния, по-другому, летнего солнцеворота. До этого момента Солнце каждый день приближалось к зениту $Z$, а в последующие дни оно будет удаляться от зенита. При нахождении Солнца $S$ в точке $E$ наибольшей будет длительность ночи. Это точка зимнего солнцестояния. А при нахождении Солнца $S$ в точках $\gamma$ или $\gamma^{\prime}$ его суточное перемещение будет происходить по кругу экватора $A A^{\prime}$. Этот круг пересекает круг горизонта $H H^{\prime}$ по его диаметру, поэтому время нахождения Солнца над горизонтом и под ним одинаково, т.е. длительность дня равна длительности ночи.

Если наблюдатель в точке $M$ на Рис. 2 будет находиться на большей широте, т.е. дуга $N_{r d} N$ будет больше, то окружность $S M_{d}$ не пересечет круг горизонта $H H^{\prime}$. В этом случае для наблюдателя $M$ наступит полярный день. При нахождении Солнца $S^{\prime}$ в южном полушарии вблизи т. $E$ круг его суточного движения также не пересечет линию горизонта $H H^{\prime}$. В этом примере широты для наблюдателя в точке $M$ наступит полярная ночь.

\section{4. Длительность солнечных суток}

Длительность солнечных суток $T_{s d}$ определяется периодом прохождения Солнца через точку полдня $M_{d}$ (Рис. 2). За время $T_{s d}$ Солнце $S$ по эклиптике $E E^{\prime}$ переместится в точку $S_{1}$ с прямым восхождением $\alpha_{s 1}=\gamma B_{1}$, которое определяется формулой (5). В связи с этим полдень наступит при часовом угле $\omega_{s d 0}<2 \pi$ на величину $\Delta \alpha_{0}=\alpha_{S 1}-\alpha_{S}$ т.е. часовой угол солнечных суток будет

$$
\omega_{s \alpha 0}=2 \pi-\Delta \alpha_{0}
$$

Как следует из (5) величина $\Delta \alpha_{0, j 1}$ определяется разностью долгот Солнца $\lambda_{j 1}$ за два соседних дня. Величина $\Delta \lambda_{j 1}$ изменяется по двум причинам: из-за неравномерного движения Солнца по эллиптической орбите и из-за наклона орбиты под углом $\varepsilon$ к плоскости экватора. Из-за наклона одинаковые дуги $S S_{1}$ на круге эклиптики $E E^{\prime}$ проектируются в неодинаковые дуги $B B_{1}$ на круге экватора $A A^{\prime}$. Разность прямых восхождений за два соседних дня запишем в виде:

$$
\Delta \alpha_{0, j 1}=\alpha_{s, j 1}-\alpha_{s, j 1-1} .
$$

Найдем среднюю за год разность прямых восхождений 


$$
\Delta \alpha_{m}=\sum_{j 1=1}^{365} \frac{\Delta \alpha_{0, j 1}}{365}
$$

Тогда по отношению к средней величине $\Delta \alpha_{m}$ разность долгот за солнечные сутки будет $\Delta \alpha=\Delta \alpha_{0}-\Delta \alpha_{m}$ и часовой угол солнечных суток запишется

$$
\omega_{s d}=2 \pi-\left(\Delta \alpha_{0}-\Delta \alpha_{m}\right) .
$$

А длительность солнечного дня в часах будет иметь вид:

$$
T_{s d}=\frac{24}{2 \pi} \omega_{s d}=12\left(2-\frac{\Delta \alpha_{0, j 1}-\Delta \alpha_{m}}{\pi}\right),
$$

где $24 / 2 \pi$-коэффициент преобразования дуги, измеряемой в радианах, в часы.

Нетрудно убедиться, что средняя за год длительность солнечных суток, согласно (12), $T_{s d m}=24$ часа. Тогда отклонение длительности солнечных суток в минутах от средних составляет:

$$
\Delta T_{\text {sd }}=60\left(T_{\text {sd }}-24\right) .
$$

Приведем некоторые значения для 2015 года. Отклонение солнечных суток от средних в день весеннего равноденствия $j_{1}=1$ будет $\Delta T_{s d}=0.297$ мин, наибольшее значение $\Delta T_{s d}=0.358$ минуты при $j_{1}=181$, и наименьший солнечный день при $j_{1}=278: \Delta T_{s d}=-0.497$ минуты. В современной цивилизации счет времени $m$ происходит по средним солнечным суткам $T_{s d m}$. Они делятся на 24 часа, 1 час состоит из 60 минут, а минута-из 60 секунд. 3а счет отклонения $\Delta T_{s d}$ солнечных суток от средних $T_{s d m}$ накапливается отличие $\eta_{0}$ солнечного времени от среднего. Последовательное суммирование отклонений запишем в виде

$$
\eta_{0, j 1}=\eta_{0, j 1-1}+\Delta T_{s d, j 1-1} .
$$

Найдем среднее отклонение за год

$$
\eta_{0 m}=\left(\sum_{j_{1}=1}^{365} \eta_{0, j 1}\right) / 365
$$

Средняя величина отклонения $\eta_{0 m}$ образуется при счете времени по средним солнечным суткам. Тогда отклонение солнечного времени от среднего солнечного времени будет

$$
\eta_{j 1}=\eta_{0, j 1}-\eta_{o m} .
$$

Величина $\eta$ в астрономии называется уравнением времени. Поэтому истинное солнечное время $m_{a}$ в часах будет выражаться через среднее солнечное время $m$ так:

$$
m_{a}=m+\eta,
$$

где $m$ - отсчитывается от полуночи.

График для уравнения времени $\eta\left(T_{d}\right)$ приведен на Рис. 3. Отклонения времени $\eta$ для дня весеннего равноденствия $j_{1}=1$ равно $\eta=-7.47$ мин, 


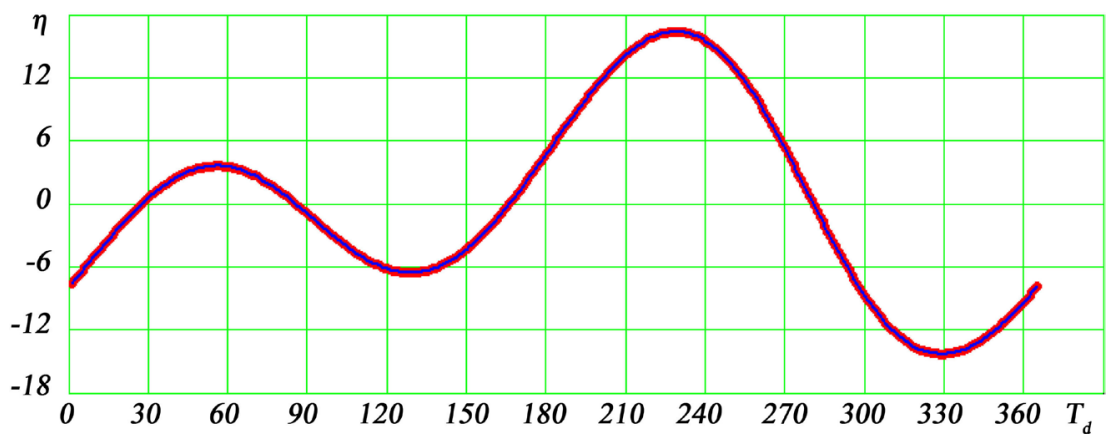

Рис. 3. Уравнение времени в современную эпоху 30.12.1949 г.: $\eta$-в мин; $T_{d}$ в днях от момента весеннего равноденствия.

наименьшее значение $\eta_{m n}=-14.25$ мин при $j_{1}=329$, а наибольшее $\eta_{m x}=$ 16.43 мин при $j_{1}=229$. Величины отклонения $\eta$ не отличаются от известных в астрономии [24]. Отличие имеется в начале отсчета времени $T_{d}$ в днях: в астрономии дни отсчитываются с 1 января, а на Рис. 3-с момента весеннего равноденствия.

Следует отметить, что по величине отклонения времени $\eta$ можно также определить отклонение истинных солнечных суток от средних

$$
\Delta T_{s d, j 1}=\eta_{j 1+1}-\eta_{j 1} \text {. }
$$

Клавдий Птолемей использовал равноденственные часы [25]. Как показано выше, весенние равноденственные сутки больше среднесолнечных на $\Delta T_{s d}=0.297$ мин. Если он и предшествующие ему астрономы использовали равноденственное время, оно может отличаться от среднесолнечного времени на величину порядка 10 минут. Роберт Ньютон [26] обвинил Клавдия Птолемея в искажении моментов древних наблюдений, потому что они не совпадают с современной теорией примерно на такое же количество минут. Как видим, одной из причин несовпадения может быть разная длительность использованных часов времени.

Длительность солнечных суток, согласно (12), определялась разностями долгот $\lambda_{j 1}-\lambda_{j 1-1}$ за средние солнечные сутки $T_{s d m}=24$ часа. Был рассчитан скорректированный ряд долгот $\lambda_{c, j l}$ по фактической длительности суток $T_{s d, j 1}$ и повторены расчеты по формулам (9)-(16). Наибольшее отличие скорректированной длительности суток $\Delta T_{s d c}$ от $\Delta T_{s d}$ равно 0.092 сек при $j_{1}$ $=278$. Это составляет относительную погрешность отклонения длительности солнечных суток $0.3 \%$. Поэтому алгоритм расчета (9)-(16) для длительности суток $T_{s d}$ и уравнения времени $\eta$ можно использовать без коррекции.

На Рис. 4 изменение длительности солнечных суток $\Delta T_{s d}$ в современную эпоху $(T=0)$ сопоставлено с этими изменениями в другие четыре эпохи. Как видно время экстремумов $\Delta T_{s d}$ примерно совпадает и приходится на моменты равноденствий ( $T_{d} \approx 0$ и $\left.T_{d} \approx 180\right)$ и солнцестояний ( $T_{d} \approx 90$ и $T_{d} \approx$ 280). При этом изменение длительности солнечных суток происходят в пределах от -0.5 мин до 0.35 мин от средней длительности в 24 часа.

В табл. 2 основные параметры уравнения времени $\eta$, представленного на 


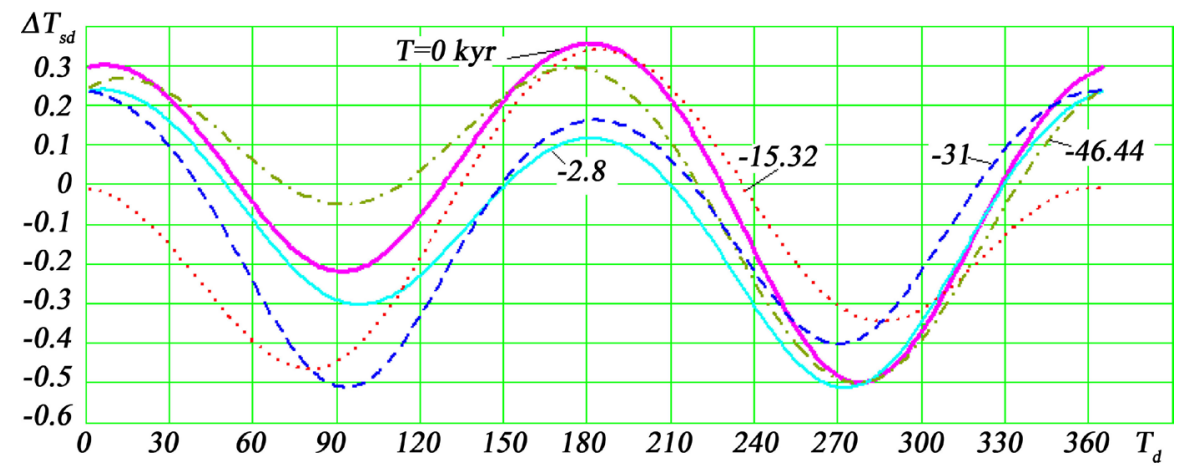

Рис. 4. Отклонение $\Delta T_{s d}$ длительности солнечных суток в эпохи $T$ от средних за год в эти же эпохи: $\Delta T_{s d}$ в минутах; $T$-время в тыс. лет от 30.12.1949 г.

Таблица 2. Изменения параметров уравнения времени по эпохам $T$ : $T$-время в тыс. лет от 30.12.1949 г.

\begin{tabular}{ccccc}
\hline \multirow{2}{*}{$T, k y r$} & $\eta_{\text {от }}$ & $\eta_{m x}$ & $\eta_{m n}$ & $\Delta \alpha_{m}$ \\
\cline { 2 - 5 } & & мин & & радианы \\
\hline 0 & 7.80093 & 16.4349 & -14.2489 & 0.0172030 \\
-2.8 & 7.21693 & 12.1048 & -18.0700 & 0.0172034 \\
-15.32 & -2.54582 & 11.8227 & -14.7360 & 0.0172024 \\
-31 & -4.69624 & 26.1632 & -19.2286 & 0.0172042 \\
-46.44 & 6.45078 & 9.00746 & -8.54319 & 0.0172025 \\
\hline
\end{tabular}

Рис. 3 для современной эпохи ( $T=0)$, сопоставлены с таковыми для других четырех эпох. Среднее отклонение $\eta_{0 m}$, согласно (15), как видно из Таблица 2 изменяется в широких пределах: от -4.7 мин в эпоху 31 т.л.н. до 7.8 мин в современную эпоху. Максимальное отклонение $\eta_{m x}$ и минимальное $\eta_{m n}$ также изменяются в 2 - 3 раза. В то же время средняя разность прямых восхождений $\Delta \alpha_{m}$ за одни сутки, согласно (10), остается неизменной $\Delta \alpha_{m}=$ 0.0172. Это является следствием неизменности периодов орбитального и вращательного движения Земли.

На Рис. 2 мы рассматривали вращения Земли со средней угловой скоростью $\vec{\omega}_{E}$. Угловая скорость Земли имеет свои независимые колебания [3] [10], но их амплитуда на три порядка меньше амплитуды изменения солнечных суток. Поэтому колебания угловой скорости вращения Земли здесь не учитываются.

\section{5. Долгота светового дня}

\section{1. Долгота светового дня в течение года}

Длительность светового дня, или долгота дня $D_{0}$ в часах определяется (см. Рис. 2) суммой часовых углов $\omega_{0}$ восходов и заходов центра Солнца (8):

$$
D_{0}=(24 / 2 \pi) \cdot 2 \omega_{0}=(24 / \pi) \cdot \omega_{0} .
$$

Так как часовой угол $\omega_{0}$ отсчитывается до центра Солнца, то величина $D_{0}$ 
определяет длительность нахождения над горизонтом $H H^{\prime}$ центра Солнца. Световой день начинается с появления края Солнца и заканчивается с опусканием его за горизонт. Кроме того, из-за уменьшения плотности атмосферы с высотой происходит преломление (рефракция) светового луча так, что Солнце видно на угол $\rho_{90}$ под горизонтом. В общем случае для наблюдаемых на небе объектов отклонение $\rho_{90}$ при зенитном угле $Z=90^{\circ}$, или рефракция, зависит от ряда факторов, которыми для Солнца можно пренебречь. Поэтому в астрономии рефракция принята постоянной и равно $\rho_{90}=34^{\prime}$ [24].

На Рис. 2 часовой угол $Z$ показан между центром Солнца и зенитом $Z$ наблюдателя $M$, находящегося на поверхности Земли. А формулой (7) угол $Z$ определяется от центра Земли. Угол $p$, под которым расстояние наблюдателя от центра Земли видно с Солнца, называется его параллаксом. Положение Солнца на небесной сфере будет отличаться на этот угол для двух наблюдателей, находящихся в точке зенита и в точке восхода, например. На угол $p$ необходимо уменьшить рассчитанную по (7) величину зенитного угла. Наибольшая величина параллакса $p$ не превышает 8".8 [24]. Эта величина в 230 раз меньше величины рефракции $\rho_{90}$, поэтому параллаксом можно пренебречь.

Имеется еще один источник искажения положения Солнца на небосводе (см. Рис. 5), который обусловлен движением наблюдателя $M$ относительно Солнца $S$ со скоростью $\vec{v}$. Это явление называется аберрацией света.

При движении наблюдателя относительно источника света $S$ происходят изменения всех характеристик света. Точное выражение для измененных характеристик света приведено в работе [27]. При скорости движения $v$, значительно меньшей скорости света $c$ для угла смещения источника справедливо выражение

$$
\begin{gathered}
\sigma=\beta \cdot \sin \theta_{o b}, \\
\text { где } \beta=v c
\end{gathered}
$$

Истинное положение источника $S$ отличается от наблюдаемого положения $S_{o b}$ на угол $\sigma$. Упрощенно, как это принято в астрономии [24], можно рассмотреть движение наблюдателя $M$ относительно Солнца за счет суточного вращения Земли $v_{r t}$ и за счет ее орбитального движения $\left(v_{o r}\right)$ относительно Солнца. При суточном вращении в точках восхода и захода Солнца углы $\theta$ между ним и скоростью $v_{r t}$ близки к 0 и $\pi$. Поэтому, согласно

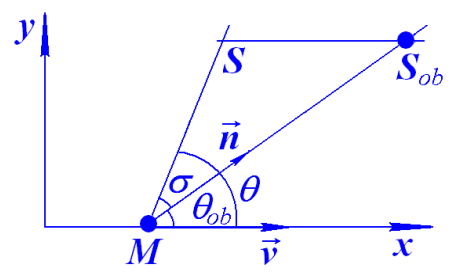

Рис. 5. Угол $\theta_{o b}$ видимого положения Солнца $S_{o b}$ при движении наблюдателя $M$ со скоростью $\vec{v}$ и угол $\theta$ действительного положения солнца $S$. 
(20), угол смещения $\sigma$ будет близкий к нулю. За счет орбитального движения в этих же точках угол $\theta$ между Солнцем и скоростью $v_{o r}$ близок к $\pi / 2$. В этом случае угол наблюдаемого Солнца будет на величину $\sigma$ меньше, чем угол истинного. Это будет приводить к более раннему восходу Солнца и более раннему его заходу. Так что на длительность светового дня это явление не оказывает влияние. При этом угол смещения $\sigma$ не превышает 20" [24]. Эта величина в 100 раз меньше величины рефракции $\rho_{90}$, а также меньше неопределенности величины $\rho_{90}$. Поэтому аберрацией света можно пренебречь.

Следует отметить, что в силу пренебрежения аберрацией света такое рассмотрение этого явления по составляющим скорости $V_{r t}$ и $V_{o r}$ допустимо. Однако при точном его рассмотрении, необходимо рассматривать полный вектор скорости, его три угла наклона к линии $M S_{o b}$ (Рис. 5). Затем вычислять изменение этих углов по точным формулам в работе [27].

Теперь рассмотрим восходы и заходы верхнего края Солнца. На Рис. 6 показано смещение края диска Солнца на угловое расстояние $G K=\rho_{90}$ ниже уровня горизонта $H H^{\prime}$ в момент его захода. Видимый радиус Солнца $K S_{s 1}$ согласно [24] равен:

$$
K S_{s 1}=\frac{961 " .18}{\rho}=\frac{4.6599 \times 10^{-3}}{\rho},
$$

где $\rho$ - расстояние Солнца от Земли, отнесенное к большой полуоси а орбиты Земли. Величина $\rho$ рассчитывается по уравнению траектории в зависимости от долготы $\lambda$ [21] [22].

С учетом (21) дуга $G S_{s 1}$ будет равна в радианах

$$
G S_{s 1}=10^{-3} \cdot\left(9.8902+\frac{4.6599}{\rho}\right) \text {. }
$$

Дуга $G S_{s 1}$ расположена на окружности большого круга, проходящего через точку зенита $Z$ (Рис. 2), поэтому она является приращением зенитного угла, т.е. $\Delta z=G S_{s 1}$. Тогда зенитный угол видимого края заходящего Солнца будет

$$
z_{0 a}=\pi / 2+G S_{s 1} .
$$

Так как часовой угол $\omega$ связан с зенитным углом $z$ выражением (7), то часовой угол видимого захода края Солнца можно записать так:

$$
\cos \omega_{0 a}=\frac{\cos z_{0 a}-\sin \delta \cdot \sin \varphi}{\cos \delta \cdot \cos \varphi} .
$$

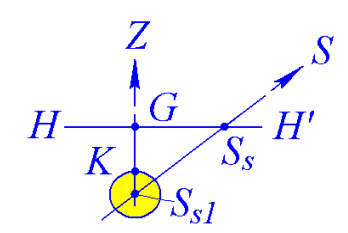

Рис. 6. Аберрационное смещение $G K=\rho_{90}$ края диска Солнца в момент его захода: $H H^{\prime}$-линия горизонта; $S_{s 1}$-положение центра Солнца во время захода его края. 
Так как правая часть по модулю может превышать 1, то перепишем выражение (24) в виде:

$$
\omega_{0 a}=\operatorname{arcos}(F n),
$$

где функция $F n$ определяется в зависимости от промежуточной функции

$$
F n 0=\frac{\cos z_{0 a}-\sin \delta \cdot \sin \varphi}{\cos \delta \cdot \cos \varphi}
$$

так:

$F n=F n 0$ при $-1 \leq F n 0 \leq 1 ; F n=-1$ при $F n 0<-1 ; F n=1$ при $F n 0>1$.

Как следует из Рис. 2, зенитный угол видимого края восходящего Солнца будет $z_{0 r a}=-\pi / 2-G S_{s 1}$, т.е. по модулю такой же, как и $z_{0 a}$. Поэтому модуль часового угла восхода Солнца будет определяться также выражением (25). Тогда долгота светового дня в радианах равна $2 \cdot \omega_{0 a}$, а в часах запишется так:

$$
D=24 \cdot \omega_{0 \alpha} / \pi
$$

На Рис. 7 показано изменение долготы дня $D$ в течение года на разных широтах. Например, на широте $\varphi=60^{\circ}$ в момент весеннего равноденствия $\left(T_{d}=1\right) D=12.31$ часа, затем она увеличивается до 18.87 часа в момент летнего солнцестояния. В момент осеннего равноденствия $D=12.26$ часа, а затем уменьшается до $D=5.87$ часа в день зимнего солнцестояния. С уменьшением широты $\varphi$ экстремумы долготы дня $D$ уменьшаются и приближаются к 12.1 часа на экваторе $\left(\varphi=0^{\circ}\right)$.

С увеличением широты $\varphi>60^{\circ}$ наибольшая долгота дня в день летнего солнцестояния приближается к 24 часам. На полярном круге $\left(\varphi=90^{\circ}-\mathcal{E}^{\circ}\right)$ наступает полярный день, который длится 31 сутки. С увеличением широты длительность полярного дня увеличивается до половины года на полюсе. В день зимнего солнцестояния долгота дня с увеличением широты уменьшается до $D=2.17$ часа на полярном круге. С дальнейшим увеличением широты наступает полярная ночь.

Представленная на Рис. 7 картина изменения долготы дня совпадает с

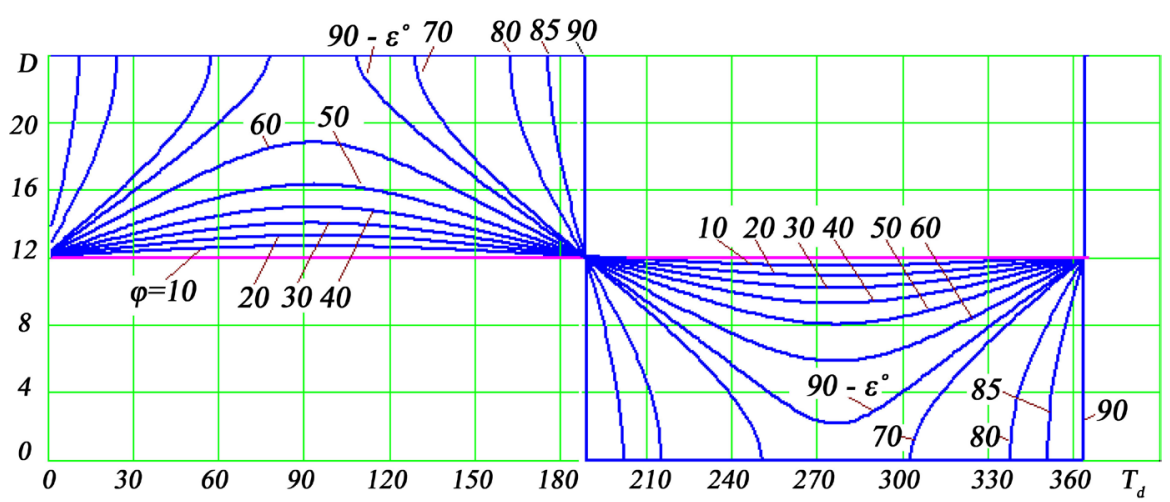

Рис. 7. Продолжительность (долгота) светового дня в году на разных широтах $\varphi$ Северного полушария в современную эпоху 30.12.1949 г. 
таковой в астрономии [24].

\section{2. Полярные дни и ночи: моменты наступления и длительность}

Рассмотрим моменты наступления полярных дней и ночей и их длительность. Для этого необходимо рассчитать долготы $\lambda$ этих событий. Как уже отмечалось, полярному дню соответствует долгота (длительность) дня $D_{d}=24$ часа, а полярной ночи $D_{n}=0$ часов. Тогда из (28) получаем часовые углы полярного дня $\omega_{0 a d}=\pi$ и полярной ночи $-\omega_{0 a n}=0$. После подстановки в (25) вместо $\omega_{0 a}$ этих значений часового угла, получаем $F n_{d}=$ -1 для полярного дня и $F n_{n}=1$ для полярной ночи. Здесь функция $F n$ определяется выражением (26). Перепишем его в следующем виде:

$$
\cos z_{0 a}-\sin \delta \cdot \sin \varphi=F n \cdot \cos \delta \cdot \cos \varphi .
$$

Подставив в это выражение значение $\sin \delta$, согласно (4), получим следующее уравнение для долготы $\lambda$

$$
\cos z_{0 a}-\sin \varepsilon \cdot \sin \lambda \cdot \sin \varphi=F_{n} \sqrt{1-\sin ^{2} \varepsilon \cdot \sin ^{2} \lambda} \cdot \cos \varphi .
$$

После возведения (29) в квадрат и преобразований с учетом того, что для рассматриваемого случая $F n^{2}=1$, получаем для $\sin \lambda$ квадратное уравнение

$$
\sin ^{2} \lambda-2 \frac{\sin \varphi \cdot \cos z_{0 a}}{\sin \varepsilon} \cdot \sin \lambda-\frac{\cos ^{2} \varphi-\cos ^{2} z_{0 a}}{\sin ^{2} \varepsilon}=0 .
$$

Оно имеет два решения, отличающиеся знаками “+” и “-” перед квадратным корнем. Запишем это решение в виде одной формулы:

$$
\sin \lambda=\text { Fn01; Fn02, }
$$

где функция со знаком “+” имеет вид:

$$
F n 01=\left[\sqrt{\left(\sin \varphi \cdot \cos z_{0 a}\right)^{2}+\cos ^{2} \varphi-\cos ^{2} z_{0 a}}+\sin \varphi \cdot \cos z_{0 a}\right] / \sin \varepsilon .
$$

Функция Fn02 перед корнем имеет знак “-”.

Чтобы исключить значения по модулю превышающие 1 вводятся функции

$$
\begin{aligned}
& F n 1=F n 01 \text { при } 1 \geq F n 01 \geq-1 ; F n 1=1 \text { при } F n 01>1 ; \\
& \text { Fn2 = Fn02 при } 1 \geq \text { Fn02 } \geq-1 ; \text { Fn2 }=-1 \text { при Fn02 }<-1 \text {. }
\end{aligned}
$$

Летнее полугодие характеризуется долготами $0<\lambda<\pi$, а зимнее $-\pi<\lambda<$ $2 \pi$. Так как функция $F n 1>0$, то она будет давать летние долготы, а отрицательная функция Fn2-зимние долготы. Тогда в соответствии с (31) летние долготы полярных дней будут $\lambda_{p d}=\arcsin (F n 1)$. Эта функция будет давать два решения: для первого $\lambda_{p d}$ и второго квадранта $\lambda_{p d 1}$. Как следует из Рис. 7 меньшее значение соответствует началу полярного дня, а большее-концу. Окончательно долготы начала и конца полярных дней запишутся в виде:

$$
\lambda_{p d}=\arcsin (F n 1) ; \lambda_{p d 1}=\pi-\arcsin (F n 1) .
$$


Аналогично долготы начала и конца полярных ночей определятся так:

$$
\lambda_{p n}=2 \pi+\arcsin (F n 2) ; \lambda_{p n 1}=\pi-\arcsin (F n 2) .
$$

Чтобы найти моменты по времени $T_{d}$ соответствующие долготам $\lambda_{p d} \lambda_{p d 1}$, $\lambda_{p n}, \lambda_{p n 1}$, необходимо найти индексы $j_{1}$ соседних долгот. Затем интерполяцией определяются моменты: $T_{d d}$ и $T_{d d 1}$-начала и конца полярного дня, а также $T_{d n}$ и $T_{d n 1}$-начала и конца полярной ночи. Алгоритм вычисления приведен в п. 11 программы SunFhnm.mcd в Приложении, а на Рис. 8 они представлены в виде графиков. В области $I$ приблизительно до $T_{d}=90$ дней изображены начала полярных дней в зависимости от широты $\varphi$. Разными линиями и точками показаны графики для 5 эпох. Аналогично в области $I I$ приблизительно при $90<T_{d}<180$ дней этими же линиями показаны моменты $T_{d}$ окончания полярных дней. Аналогично приблизительно при $180<T_{d}<270$ и $T_{d}>270$ изображены начала (III) и окончания (IV) полярных ночей.

Например, в современную эпоху $T=0$ на широте $\varphi=70^{\circ}$ в $T_{d}=56.88$ день от момента весеннего равноденствия наступает полярный день. Он заканчивается в $T_{d}=128.82$ день. Полярная ночь наступает в $T_{d}=250.05$ день и заканчивается в $T_{d}=302.39$ день. С увеличением широты $\varphi$ начала полярных дней и ночей происходит раньше, а окончание позже. С уменьшением широты $\varphi$ начала полярных дней отдаляются и приближаются к $T_{d}=92.8$ дню, а начала полярных ночей приближаются к $T_{d}=275.5$ дню. Это происходит на широтах близких к широте полярного круга, которая для современной эпохи равна $66.56^{\circ}$.

В другие эпохи графики начала и конца полярных дней и ночей, как видно из Рис. 8, идентичны, но широта их начала может существенно смещаться. При этом изменяется продолжительность полярных дней и ночей. Например, в эпоху 31 т. л. н. широта начала полярных дней и ночей

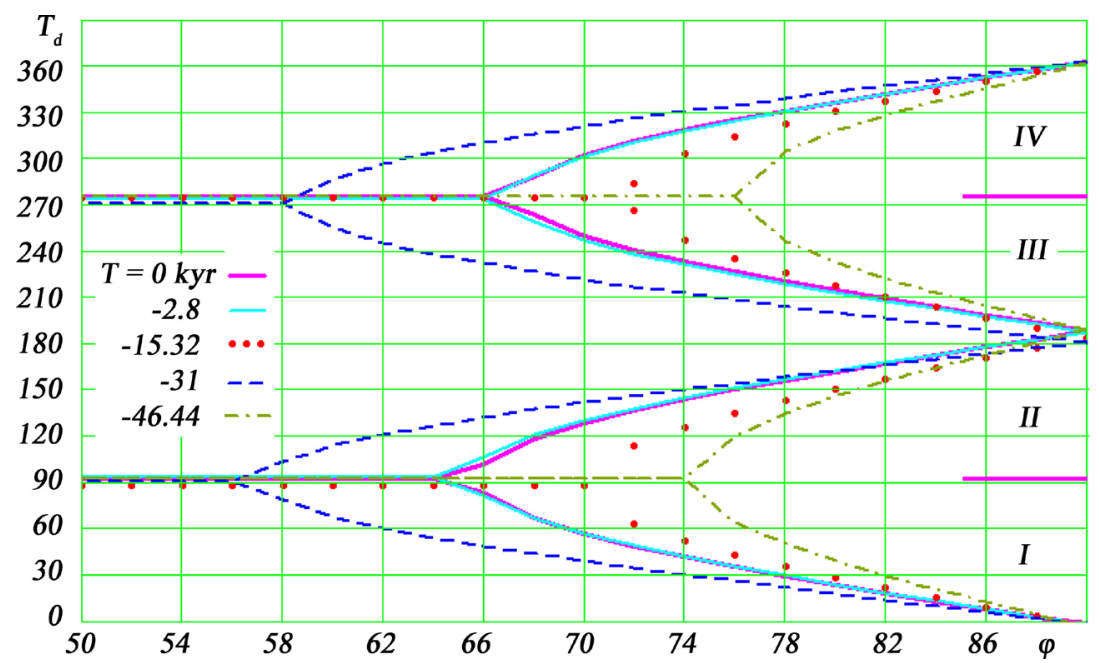

Рис. 8. Моменты $T_{d}$ наступления $(I, I I)$ и окончания $(I I, I V)$ полярных дней $(I, I I)$ и ночей $(I I I, I V)$ на разных широтах Северного полушария в экстремальные эпохи за последние 50 т.л.н. 
смещается до56 ${ }^{\circ}$ и $58^{\circ}$, соответственно, а в эпоху 46.44 т. л. н.-до $74^{\circ}$ и $76^{\circ}$ соответственно.

Следует отметить, что древние арии [15] в эпоху 31 т. л. н. могли обитать на территории близкой по широте г. Тюмени $\left(\varphi=57.15^{\circ}\right)$ и наблюдать полярные дни и ночи. С приближением последнего ледникового периода 15.32 т. л. н. они вынуждены были мигрировать на южные территории.

Начала полярных дней и ночей и их продолжительность $\Delta T_{d d}=T_{d d 1}-T_{d d}$ и $\Delta T_{d n}=T_{d n 1}-T_{d n}$ соответственно, на разных широтах рассчитаны для пяти разных эпох. Они представлены в табл. 1П в Приложении работы [28], которая имеется в свободном доступе. Для сравнения в этой таблице приведены длительности полярных дней и ночей в астрономии в современную эпоху. Они обозначены как $E A$ по названию “Эфемеридная астрономия" источника [24]. Данные $E A$ приведены с точностью 1 день. Округление начала и конца полярных дней и ночей может приводить к разности их длительности в 2 дня. В табл. 1П такое отличие наблюдается. А в целом представленные на Рис. 8 и табл. 1П результаты для современной эпохи совпадают с известными данными в астрономии.

\section{3. Долготы светового дня в моменты солнцестояний}

Рассмотрим долготу светового дня в моменты летнего и зимнего солнцестояний в неполярных широтах. Как уже отмечалось, наибольшая долгота светового дня будет в момент летнего солнцестояния $\lambda_{s}=\pi / 2$, a наименьшая - в момент зимнего солнцестояния $\lambda_{w}=1.5 \cdot \pi$. Тогда склонение Солнца $\delta$, согласно (4), в этих случаях будет $\delta_{s}=\varepsilon$ и $\delta_{w}=-\mathcal{E}$, соответственно. Тогда после подстановки угла $\delta_{s}$ в $(26)$ часовой угол видимых восходов и заходов края Солнца в момент летнего солнцестояния запишется аналогично (25)-(27) так:

$$
\begin{gathered}
\omega_{0 \text { sa }}=\operatorname{arcos}\left(F n_{s}\right) ; \\
F n 0_{s}=\frac{\cos z_{0 a 1}-\sin \varepsilon \cdot \sin \varphi}{\cos \varepsilon \cdot \cos \varphi} ; \\
F n_{s}=F n 0_{s} \text { при }-1 \leq F n 0_{s} \leq 1 ; F n_{s}=-1 \text { при } \\
F n 0_{s}<-1 ; F n_{s}=1 \text { при } F n 0_{s}>1 .
\end{gathered}
$$

В формуле (30) использован зенитный угол $z_{0 a 1}$, в котором безразмерное расстояние от Земли до Солнца $\rho$, согласно (22), из-за малого влияния его изменения принято $\rho=1$.

После подстановки угла $\delta_{w}=-\varepsilon$ в (26) часовой угол видимых восходов и заходов края Солнца в момент зимнего солнцестояния запишется аналогично (37)-(39) так:

$$
\begin{gathered}
\omega_{0 w a}=\operatorname{arcos}\left(F n_{w}\right) ; \\
F n 0_{w}=\frac{\cos z_{0 a 1}+\sin \varepsilon \cdot \sin \varphi}{\cos \varepsilon \cdot \cos \varphi} ; \\
F n_{w}=F n 0_{w} \text { при }-1 \leq F n 0_{w} \leq 1 ; \quad F n_{w}=-1 \text { при }
\end{gathered}
$$




$$
F n 0_{w}<-1 ; F n_{w}=1 \text { при } F n 0_{w}>1 .
$$

Тогда долгота светового дня в моменты летнего и зимнего солнцестояний запишутся, соответственно, так:

$$
D_{s}=24 \cdot \omega_{0 s \alpha} / \pi ; \quad D_{w}=24 \cdot \omega_{0 w \alpha} / \pi .
$$

Результаты расчетов долготы дня в моменты солнцестояний по формулам (37)-(43) на Рис. 9 даны для пяти разных эпох. Графики представляют изменение долготы дня $D$ по широтам $\varphi$ северного полушария. Для зимнего солнцестояния долгота дня $D \leq 12$ часов отмечена как область $I$, а для летнего солнцестояния-как область $I I$.

В современную эпоху, как видно из Рис. 9, на широте $50^{\circ}$ в зимнее солнцестояние день длится 8 часов, а в летнее-16.37 часа. С уменьшением широты день в зимнее солнцестояние увеличивается и приближается к 12 часам на экваторе. А в летнее солнцестояние день уменьшается и на экваторе также приближается к 12 часам. С увеличением широты $\varphi$ долгота зимнего дня уменьшается и приближается к нулю. То есть наступает полярная ночь, а долгота летнего дня увеличивается до 24 часов и наступает полярный день.

Для остальных эпох изменения долготы дня изменяются подобным образом, но с разными наклонами зависимостей $D(\varphi)$. Например, для эпохи 31 т. л. н. полярные ночи и дни начинаются на меньших широтах, а в эпоху 46.44 т. л. н.-на больших широтах.

В табл. 2П работы [28] приведены долготы дней для летнего солнцестояния $\left(D_{s}\right)$ и зимнего $\left(D_{w}\right)$ для пяти разных эпох. Изменение широты $\varphi$ дается через $2^{\circ}$, начиная с экватора. Широты изменяются до величин, после которых наступает полярный день $\left(D_{s}=24\right.$ часа) и полярная ночь $\left(D_{w}=0\right.$ часов).

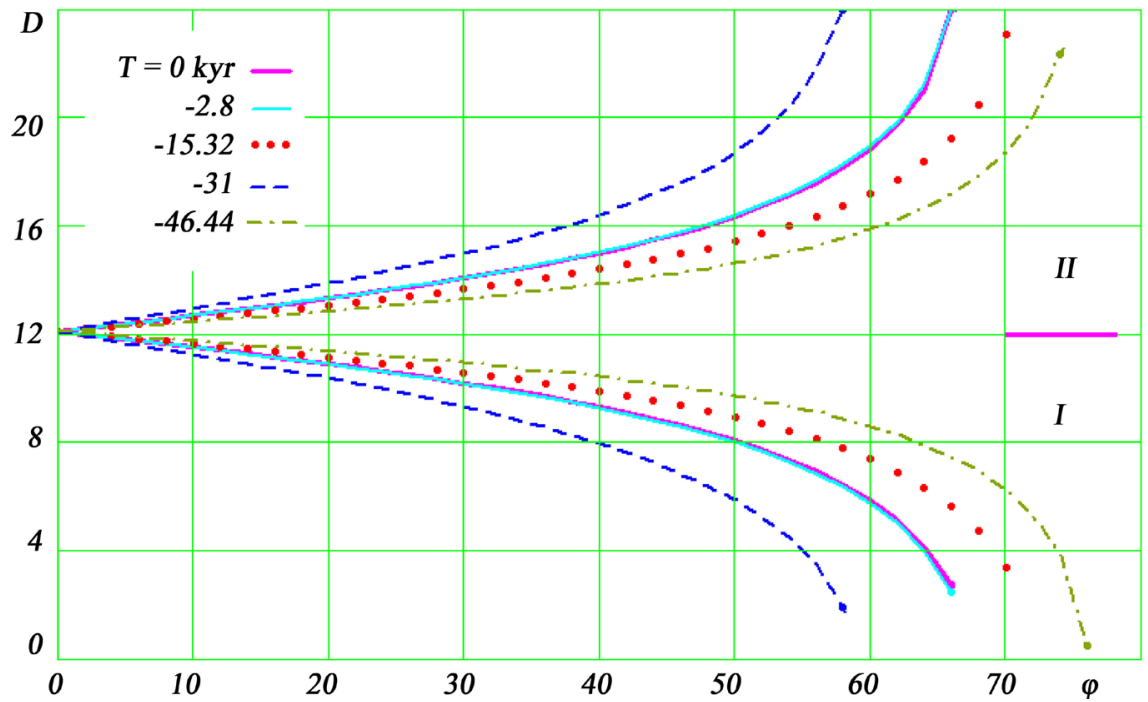

Рис. 9. Долгота светового дня $D$ в моменты зимнего солнцестояния (I) и летнего солнцестояния (II) на разных широтах $\varphi^{\circ}$ Северного полушария в экстремальные эпохи $T$ за последние 50 т.л.н.: $T$-время в тыс. лет от 30.12 .1949 г.; $D$ 一долгота светового дня в часах. 


\section{6. Азимуты Солнца}

\section{1. Азимуты движения Солнца в течение суток}

Азимут Солнца $A_{S}$ отсчитывается на круге горизонта $H H^{\prime}$ (Рис. 2) от точки севера $N_{r d}$ до проекции Солнца в т. $D$ за часовой стрелкой

$$
A_{S}=N_{r d} S_{t h}+S_{t h} D=2 \pi-D N_{r d} .
$$

В треугольнике $N_{r d} Z D$, две стороны которого $N_{r d} Z$ и $Z D$ равны $\pi / 2$, дуга $D N_{r d}$ равняется углу $Z$. В треугольнике $N Z S$ известны две стороны: $N S=\pi / 2$ $-\delta$ и $Z S=Z$, а также угол $N=\omega$. По теореме синусов

$$
\sin Z / \sin N S=\sin N / \sin Z S,
$$

находим

$$
\sin Z=\sin \omega \cdot \cos \delta / \sin Z,
$$

где дуга $Z$ является зенитным углом Солнца и согласно (7) определяется выражением:

$$
z=\operatorname{arcos}(\sin \delta \cdot \sin \varphi+\cos \delta \cdot \cos \varphi \cdot \cos \omega) .
$$

Тогда с учетом (45) азимут Солнца (44) $A_{S}=2 \pi-Z$ запишется в радианах так:

$$
A_{S, r a d}=2 \pi-\arcsin (\sin \omega \cdot \cos \delta / \sin z)
$$

Так как функция $\arcsin$ в (47) неоднозначна и после достижения экстремальных значений $\pm \pi / 2$ имеет изломы, то проводятся следующие операции. Вводится функция

$$
F n 03=\arcsin (\sin \omega \cdot \cos \delta / \sin z) .
$$

Находятся максимальные значения $m x F n$ функции $F n 03$ и минимальные ее значения $m n F n$. При изменении часового угла $\omega$ от $-\pi$ до $\pi$ определяются индексы часового угла in9, соответствующего $m n F n$, и in 10 , соответствующего $m x F n$. В зависимости от этих индексов рассчитываются диапазоны индекса $k$ часового угла $\omega_{k}$ и значения функции Fn3 так:

$$
\begin{gathered}
\text { Fn3 =Fn03 при ind } \leq \leq \leq \operatorname{in10;~Fn3~}=-\pi-\text { Fn03 при } k<\text { in9; } \\
\text { Fn3 }=\pi-\text { Fn03 при } k>\text { in10. }
\end{gathered}
$$

Как видно из (48) при $\omega=0$, в полдень, функция Fn03 может быть равной 0 или $\pi$. Согласно Рис. 2 в полдень $F n 03=N_{r d} D=\pi$. Тогда с учетом этого и (48)-(49) азимут Солнца в градусах в соответствии с (47) запишется так:

$$
A_{S}=180^{\circ}+\left(180^{\circ} / \pi\right) \cdot F n 3
$$

Следует отметить, что минимальное и максимальные значения Fn03 могут принадлежать не центральному участку. В этом случае на азимуте $A_{S}$ при большом интервале между делениями $\omega$ будут всплески при переходе через $0.5 \cdot \pi$ и $1.5 \cdot \pi$. В этом случае нужно использовать следующее условие:

Fn3 $=$ Fn03 при ind $<k<$ in10; Fn3 $=-\pi-$ Fn03 при $k \leq$ in9; Fn3 $=\pi-$ 
Fn03 при $k \geq \operatorname{in} 10$.

Этот алгоритм реализован в п. 13 программы SunPhenmn.mcd. На Рис. 10 приведены азимуты движения Солнца по небосводу на широте Москвы в современную эпоху. Линией 1 показано изменение азимута в день весеннего равноденствия. Азимут Солнца в полночь $\omega=-12$ часов равен нулю и увеличивается на рассвете $\left(\omega \approx-6\right.$ часов) до $90^{\circ}$. В течение дня продолжает увеличиваться: в полдень ( $\omega=0$ часов) до $180^{\circ}$ и в полночь $(\omega$ $=12$ часов) достигает значения $360^{\circ}$. Вертикальными линиями показаны часы восхода и захода Солнца.

В дни солнцестояний: летнего (линия 2) и зимнего (линия 3) изменение азимутов происходит аналогичным образом, но границы дня существенно изменяются. Кроме того, по сравнению с изменением азимута в дни равноденствия, в эти дни характер изменения азимутов более существенно отличается от линейного закона. Нелинейный характер изменения $A_{S}$ свидетельствует, что определение промежутков времени по азимуту Солнца или по тени гномона, будет характеризоваться соответствующей неравномерностью хода времени. На Рис. 10 часовые промежутки нанесены кружками. Так как в дни равноденствий азимут $A_{S} 1$ изменяется наиболее близко к линейному закону, то равноденственные часы наиболее равномерны.

Условия (49) при вычислении азимута Солнца справедливы для нетропических широт. В тропических широтах Северного полушария широта $\varphi$ меньше угла наклона $\varepsilon$, т.е. $\varphi-\varepsilon<0$. Для зенитного угла $Z$ в полдень $(\omega=0)$ из (7) следует

$$
z_{n}=\varphi-\delta
$$

Поэтому, как уже отмечалось, в день летнего солнцестояния ( $\delta=\varepsilon$ см.

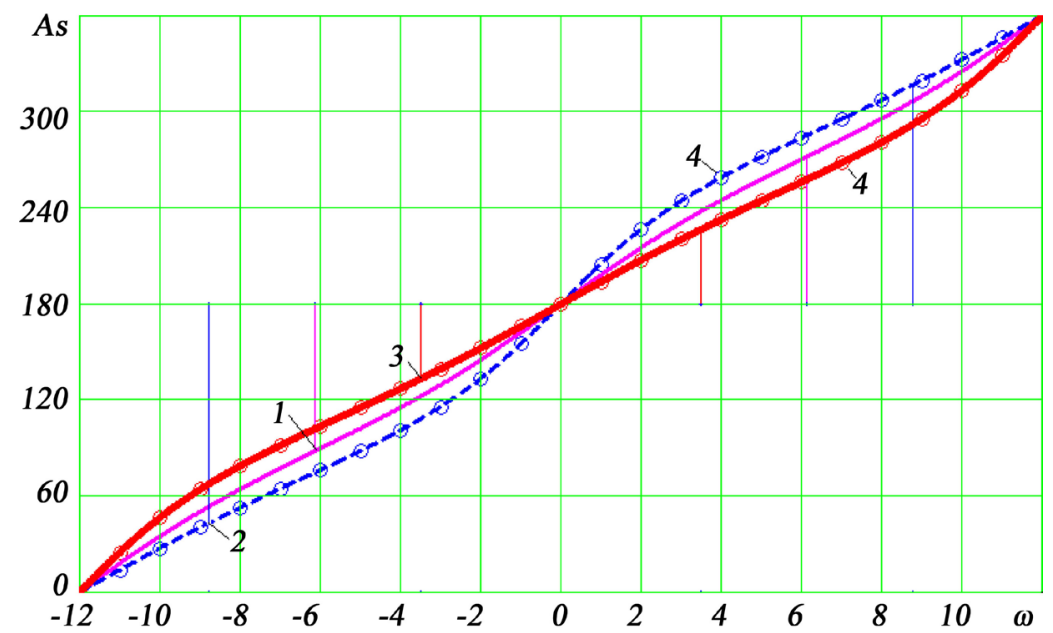

Рис. 10. Азимуты $A_{S}$ на широте Москвы $\left(\varphi^{\circ}=55.7522^{\circ}\right)$ суточного движения Солнца в дни весеннего равноденствия (1), летнего (2) и зимнего (3) солнцестояний в современную эпоху 30.12.1949 г. Вертикальными линиями отмечены часовые углы восходов $(\omega<0)$ и заходов Солнца $(\omega>0)$. Кружками 4 отмечены часовые промежутки времени. 
Рис. 2) зенитный угол в тропических широтах изменяется от

$$
z_{n s}=\varphi-\varepsilon
$$

до

$$
z_{n w}=\varphi+\varepsilon
$$

в день зимнего солнцестояния $(\delta=-\varepsilon)$. Из (52) видно, что в день летнего солнцестояния зенитный угол $Z_{n s}$ становится отрицательным, т.е. для наблюдателя $M$ (см. Рис. 2) Солнце находится в северной стороне неба. В этом случае в полдень $(\omega=0)$ азимут Солнца $A_{S n}=0$, а не $\pi$, как в нетропических широтах. Следует отметить, что в этом случае суточное движение Солнца для наблюдателя $M$ происходит на южной стороне неба справа налево, а не как на северной стороне слева направо, как это наблюдается в нетропических широтах.

Ситуации с отрицательным углом $Z_{n}$, согласно (51), имеют место не только для дня летнего солнцестояния, но и для других дней. Поэтому необходимо определять номера дней, для которых $Z_{n}<0$. Тогда для рассмотренного случая тропических широт азимут Солнца запишется так:

$$
A_{S t}=(180 / \pi) \cdot F n 03 \text { при } Z_{n}<0 .
$$

Полностью алгоритм расчета азимута Солнца приведен в п. 13 программы SunPhenmn.mcd в Приложении. На Рис. 11 представлено изменение азимута Солнца в течение суток на широте $\varphi=20^{\circ}$. В день весеннего равноденствия 1 и зимнего солнцестояния 3 азимут Солнца изменяется от $180^{\circ}$ в полночь ( $\omega=-12$ часов) до некоторого минимального значения. Для зимнего солнцестояния 3 оно приходится на ночь, а для весеннего равноденствия-на рассвет. В полдень азимут Солнца снова равняется $180^{\circ}$, т.е. Солнце находится в южной стороне неба. При движении Солнца от полдня к полночи оно проходит максимальные значения азимута и в полночь снова приходит на юг $\left(A_{S}=180^{\circ}\right)$.

В день летнего солнцестояния (линия 2, правая шкала графика) Солнце в

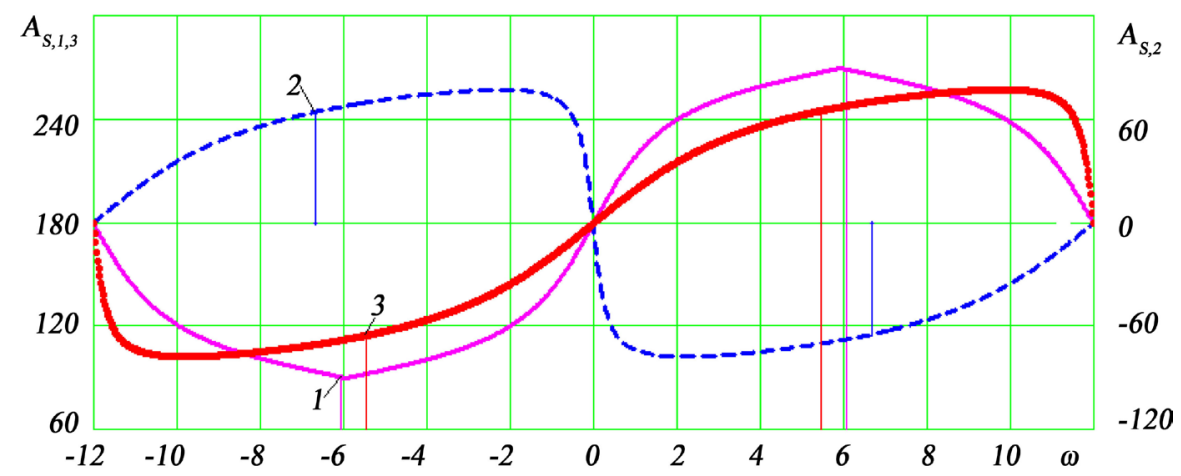

Рис. 11. Азимуты в тропических широтах $\left(\varphi^{\circ}=20^{\circ}\right)$ суточного движения Солнца в дни весеннего равноденствия 1, летнего 2 и зимнего 3 солнцестояний в современную эпоху 30.12.1949 г. Шкала азимута дня летнего солнцестояния 2 сдвинута на $180^{\circ}$ (см. справа графика). Вертикальными линиями отмечены часовые углы восходов $(\omega<0)$ и заходов Солнца $(\omega>0)$. 
полночь находится на Севере $\left(A_{S}=0^{\circ}\right)$, затем движется по северной стороне неба $\left(A_{S}<90^{\circ}\right)$. Перед полднем $(\omega \approx 1$ час) достигает максимального отклонения к востоку и в полдень возвращается на север $\left(A_{S}=0^{\circ}\right)$. После полдня Солнце переходит в западную сторону неба и достигает максимального отклонения к западу $\left(A_{\mathcal{S}} \approx 70^{\circ}\right)$. К полночи Солнце снова возвращается на север $\left(A_{S}=0^{\circ}\right)$. Следует отметить, что здесь мы использовали для определения азимута шкалу не от 0 до $360^{\circ}$, а от $-180^{\circ}$ до $180^{\circ}$. Это позволяет избежать разрыва графика на интервале часового угла $\omega$ от -12 часов до +12 часов.

В нетропических широтах суточное движение Солнца происходит по полному кругу вокруг наблюдателя $M$, т.е. $A_{S}=0 \div 2 \pi$. В тропических широтах суточный круг движения Солнца происходит в стороне от наблюдателя: летом (линия 2) в северной стороне неба, зимой, включая дни равноденствия, (линии 1 и 3) - в южной стороне неба.

\section{2. Проекция дневного пути Солнца на плоскости горизонта}

Рассмотрим проекцию на плоскость горизонта дневное движение Солнца. Положение Солнца $S$ (Рис. 2) над плоскостью горизонта $H H^{\prime}$ определяется зенитным углом $Z$ и углом азимута $A_{S}$ который определяется дугой $N_{r d} S_{r} S_{t h} D$. На плоскости горизонта введем две оси координат с началом в точке $M: N_{S}$ - направленная на Север $N_{r d}$ и $E_{S}$ - направленная на Восток $E_{s t}$ Тогда проекции центра Солнца на эти оси запишутся так:

$$
N_{S}=\sin z \cdot \cos A_{S} ; \quad E_{S}=\sin z \cdot \cos A_{S},
$$

где $A_{S}$ - азимут Солнца в радианах.

Выражением (55) также определяются координаты $N_{S} E_{S}$ точек восхода и захода верхнего края Солнца, если углами $Z$ и $A_{S}$ будут определены их моменты времени. На Рис. 12 проекции дневного пути Солнца показаны для трех случаев: в день весеннего равноденствия и в дни солнцестояний на широте Москвы.

В день весеннего равноденствия центр Солнца всходит на Востоке и заходит на Западе. А восход и заход верхнего края Солнца немного сдвигается на Север. Летом восход и заход Солнца находится в северной части горизонта. С увеличением широты $\varphi$ траектория 2 Солнца будет приближаться к окружности. На широте, где траектория станет окружностью, наступит полярный день.

В день зимнего солнцестояния траектория 3 Солнца укорачивается и находится в южной стороне неба. С увеличением широты длина ее уменьшается, и там, где траектория исчезает, наступает полярный день.

Следует отметить, что конечные точки на траектории Солнца в дни равноденствий 1 и в дни солнцестояний 2 и 3 играют ключевую роль в древных астрономических календарях [17] [18]. Представленный в п. 15 программы на SunPhnmen.mcd алгоритм позволяет рассчитать их для любой эпохи. 


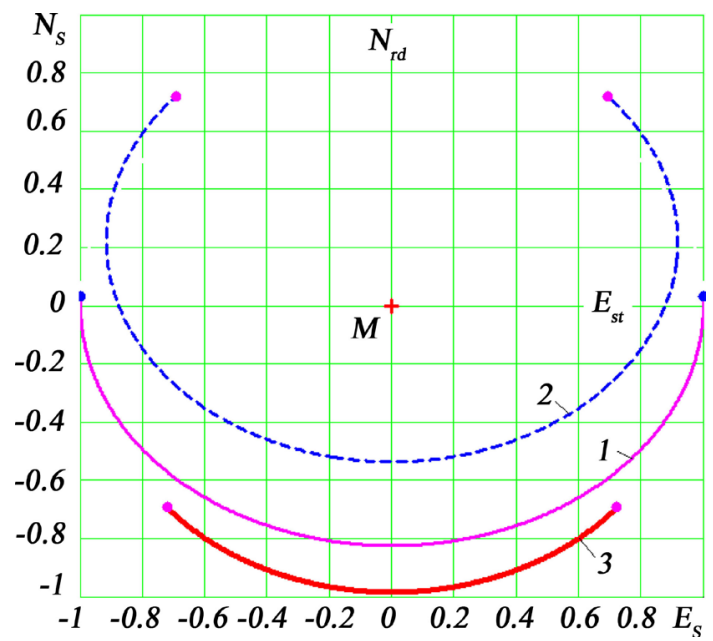

Рис. 12. Проекция дневного пути Солнца на плоскости горизонта на широте Москвы $\left(\varphi=55.7522^{\circ}\right)$ в современную эпоху 30.12 .1949 г.: 1 -в дни весеннего равноденствия; 2-летнего и 3-зимнего солнцестояний. Ось $N_{S}$ направлена на Север $\left(N_{r d}\right)$, а ось $E_{S}$-на восток $\left(E_{s t}\right)$. Положение наблюдателя $(M)$ в начале координат отмечено крестиком. Точками на линиях отмечены восходы верхнего края Солнца (с правой стороны) и его заходы (с левой стороны).

\section{3. Азимуты восходов и заходов Солнца}

В отличие от азимутов суточного движения Солнца азимуты точек восхода и захода необходимо отсчитывать до точек появления и исчезновения верхнего края Солнца. Как видно из Рис. 2 и Рис. 6, азимут наблюдаемой точки $G$ захода края Солнца состоит из дуг на круге горизонта $H H^{\prime}$

$$
A_{G s}=N_{r d} E_{s t} S_{t h} W_{s t}+W_{s t} S_{s}+S_{s} G,
$$

где $E_{s t}$ и $W_{s t}$ - точки Востока и Запада на круге горизонта.

Дуга $N_{r d} E_{s t} S_{t h} W_{s t}$ равна $1.5 \cdot \pi$ (см. Рис. 2). Через точку $S_{s}$ и полюс $N$ проведена дуга большого круга $S_{s} F$, поэтому она перпендикулярна дуге экватора $A A$, т.е. угол $F=0.5 \cdot \pi$. Дуга $S_{s} F$ равна $\delta$. В треугольнике $S_{s} F W_{s t}$ угол $W_{s t}=\pi / 2-\varphi$. Поэтому дуга $W_{s t} S_{s}$ определяется по теореме синусов

$$
W_{s t} S_{s}=\arcsin (\sin \delta / \cos \varphi) \text {. }
$$

Сторона $G S_{s}$ может быть найдена из треугольника $G S_{s 1} S_{s}$ (см. Рис. 6), в котором угол $S_{s}=W_{s t}=\pi / 2-\varphi$. В виду малости треугольника, его можно рассматривать как плоский и линейный со сторонами $R_{E} \cdot G S_{s 1}$ и $R_{E} \cdot G S_{s}$, где $R_{E}$-радиус Земли. Для угла $S_{s}$ можно записать: $\operatorname{tg}(\pi / 2-\varphi)=R_{E} \cdot G S_{s 1}\left(R_{E} \cdot G S_{s}\right)$. Отсюда получаем:

$$
G S_{s}=G S_{s 1} \cdot \operatorname{tg} \varphi
$$

Подставляя в (56) составляющие получим азимут точки захода Солнца в градусах в виде:

$$
A_{G s}=(180 / \pi) \cdot\left(1.5 \pi+\arcsin (\sin \delta / \cos \varphi)+G S_{s 1} \cdot \operatorname{tg} \varphi\right) .
$$

Ввиду того, что $\sin \delta / \cos \varphi$ по модулю может превышать 1 , введем обозначение: 


$$
F n 04=\sin \delta / \cos \varphi
$$

и определим функцию:

$$
\begin{gathered}
\text { Fn4 Fn04 при }-1 \leq \text { Fn04 } \leq 1 ; \text { Fn } 4=-1 \\
\text { при Fn04<-1; Fn4=1 при Fn04 >1. }
\end{gathered}
$$

Тогда азимут точки захода Солнца будет:

$$
A_{G s}=(180 / \pi) \cdot\left(1.5 \pi+\arcsin (F n 4)+G S_{s 1} \cdot \operatorname{tg} \varphi\right) .
$$

Чтобы рассчитать азимут точки восхода, обозначим точку $S_{r 1}$ видимого восхода края Солнца на дуге $M_{n} S_{r}$ (на Рис. 2 эта точка не приведена). Она находится под горизонтом $H H^{\prime}$ и дуга $S_{r 1} S_{r}$ равна по величине дуге $S_{s 1} S_{s}$ (см. Рис. 6), т.е. $S_{r 1} S_{r}=S_{s 1} S_{s}$. Поэтому точка наблюдаемого восхода края Солнца $G_{s}$ будет ближе к точке севера $N_{r d}$ на величину дуги $S_{r} G_{1}=S_{s} G$. Тогда азимут наблюдаемой точки $G_{s}$ восхода будет определяться следующими дугами:

$$
A_{G r}=N_{r d} E_{s t}-E_{s t} S_{r}-S_{r} G_{r},
$$

где точка $E_{s t}$ находится в пересечении кругов горизонта $H H^{\prime}$ и экватора $A A^{\prime}$, а ее азимут $N_{r d} E_{s t}=\pi / 2$. Дуга $E_{s t} S_{r}=W_{s t} S_{s}$. Подставляя в (63) все составляющие азимут точки восхода края Солнца с учетом (60)-(61) получаем в виде:

$$
A_{G r}=(180 / \pi) \cdot\left(0.5 \pi-\arcsin (F n 4)-G S_{s 1} \cdot \operatorname{tg} \varphi\right) .
$$

Как видим из (62) и (64) сумма азимутов восходов и заходов

$$
A_{G s}+A_{G r}=360^{\circ} \text {. }
$$

Сумма $A_{G s}+A_{G r}$ может отличаться от $360^{\circ}$, если горизонт в восточной части отличается от горизонта в западной части высотой окружающей местности. Приведенные формулы для азимутов не учитывают этой особенности и дают симметричные азимуты восходов и заходов относительно направления $M N_{r d}$ на север (Рис. 2).

На Рис. 13 показано изменение азимута точки восхода края Солнца $A_{G r}$ в течение года на разных широтах. Например, на широте $\varphi=60^{\circ}$ в момент весеннего равноденствия $\left(T_{d}=1\right)$ азимут $A_{G r}=87.76^{\circ}$, затем он уменьшается до $35.84^{\circ}$ в день летнего солнцестояния. В момент осеннего равноденствия $A_{G r}=88.23^{\circ}$, а затем увеличивается до $A_{G r}=141.27^{\circ}$ в день зимнего солнцестояния.

С уменьшением широты $\varphi$ экстремумы азимута Солнца $A_{G r}$ уменьшаются и на экваторе $\left(\varphi=0^{\circ}\right)$ приближаются к $66.56^{\circ}$ в день летнего солнцестояния и к $113.44^{\circ}$ в день зимнего солнцестояния. С увеличением широты $\varphi>60^{\circ}$ наименьший азимут точки восхода края Солнца $A_{G r}$ в день летнего солнцестояния приближается к $0^{\circ}$, т.е. Солнце восходит на Севере. На полярном круге $\left(\varphi=90^{\circ}-\varepsilon^{\circ}\right)$ наступает полярный день. С увеличением широты в день зимнего солнцестояния $\left(T_{d}=276\right)$ азимута Солнца $A_{G r}$ увеличивается до $180^{\circ}$ на полярном круге $\left(\varphi=90^{\circ}-\varepsilon^{\circ}\right)$, т.е. Солнце всходит на Юге. С дальнейшим увеличением широты наступает полярная ночь. 


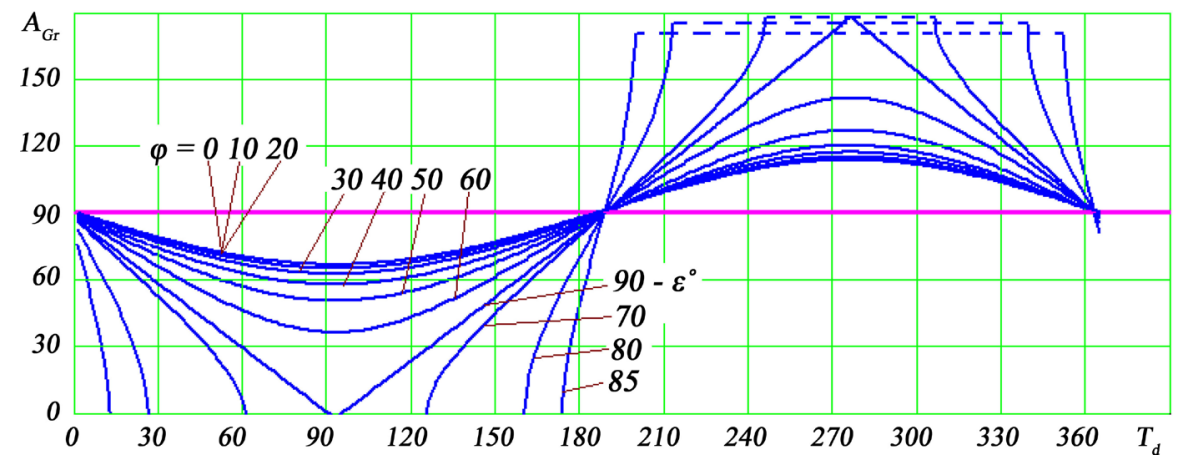

Рис. 13. Азимуты восходов верхнего края Солнца в современную эпоху 30.12.1949 г. в течение года на разных широтах $\varphi^{\circ}$ Северного полушария: $A_{G r}$-азимуты восходов Солнца в градусах.

Следует отметить, что графики на Рис. 13 напоминают графики долготы светового дня на Рис. 7, если их перевернуть вокруг горизонтальной линии $A_{G r}=90^{\circ}$. Азимуты точки захода края Солнца $A_{G s}$ как следует из (65), $A_{G s}=$ $360^{\circ}-A_{G r}$ поэтому их графики будут похожи на графики долготы светового дня на Рис. 7.

\section{4. Экстремальные азимуты восходов и заходов Солнца в дни солнцестояний}

Наибольшие азимуты заходов и наименьшие азимуты восходов верхнего края Солнца имеют место в день летнего солнцестояния $\lambda_{s}=\pi / 2$. В день зимнего солнцестояния $\lambda_{s}=1.5 \cdot \pi$ экстремумы азимутов становятся обратными: азимуты заходов-наименьшие, а азимуты восходов-наибольшие. Приведем предельные азимуты восходов (64) в день летнего солнцестояния:

$$
\begin{aligned}
A_{\text {Grsm }}= & (180 / \pi) \cdot(0.5 \pi-\arcsin (\sin \varepsilon / \cos \varphi) \\
& \left.-\left(9.8902 \times 10^{-3}+4.6599 \times 10^{-3} / \rho_{s}\right) \cdot \operatorname{tg} \varphi\right)
\end{aligned}
$$

и в день зимнего солнцестояния:

$$
\begin{aligned}
A_{\text {Grwn }}= & (180 / \pi) \cdot(0.5 \pi+\arcsin (\sin \varepsilon / \cos \varphi) \\
& \left.-\left(9.8902 \times 10^{-3}+4.6599 \times 10^{-3} / \rho_{w}\right) \cdot \operatorname{tg} \varphi\right)
\end{aligned}
$$

Азимуты заходов Солнца (62) в дни солнцестояний запишутся аналогичным образом. При вычислениях по формулам (66)-(67) в алгоритм необходимо ввести условия, чтобы избежать $|\sin \varepsilon / \cos \varphi|>1$ (см. п. 14 программы в Приложении).

Результаты расчетов азимуты восходов Солнца в дни солнцестояний по формулам (66) - (67) для пяти разных эпох даны на Рис. 14. Графики представляют изменение азимута $A_{G r}$ по широтам $\varphi$ Северного полушария. Для летнего солнцестояния азимуты $A_{G r} \leq 90^{\circ}$ отмечены как область $I$, а для зимнего солнцестояния-как область $I I$.

В современную эпоху, как видно из Рис. 14, на широте $50^{\circ}$ в летнее 


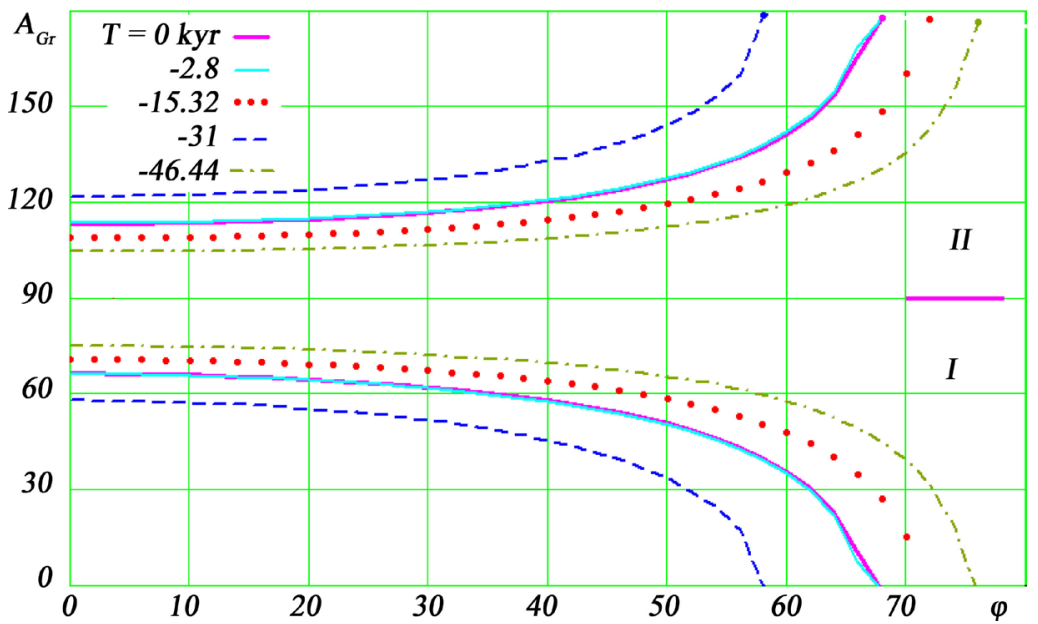

Рис. 14. Азимуты восходов верхнего края Солнца в моменты летнего солнцестояния (I) и зимнего солнцестояния (II) на разных широтах $\varphi^{\circ}$ Северного полушария в экстремальные эпохи $T$ за последние 50 т.л.н.: $T$-время в тыс. лет от 30.12.1949 г.; $A_{G r}$-азимуты восходов Солнца в градусах.

солнцестояние азимут восхода Солнца $A_{G r}$ равен $50.77^{\circ}$, а в зимнее $-127.24^{\circ}$. С уменьшением широты азимут восхода увеличивается и приближается к $66.56^{\circ}$ на экваторе. А в зимнее солнцестояние азимут восхода уменьшается и на экваторе приближается к $113.44^{\circ}$. С увеличением широты $\varphi$ азимут восхода $A_{G r}$ дня летнего солнцестояния уменьшается и приближается к нулю. То есть Солнце всходит на Севере и наступает полярный день. А азимут восхода $A_{G r}$ дня зимнего солнцестояния с увеличением широты $\varphi$ увеличивается до $180^{\circ}$ и наступает полярная ночь.

Для остальных эпох изменения азимута восхода Солнца $A_{G r}$ происходит подобным образом, но с разными наклонами зависимостей $A_{G r}(\varphi)$. Например, для эпохи 31 т. л. н. полярные ночи и дни начинаются на меньших широтах, а в эпоху 46.44 т. л. н. - на больших широтах.

Следует отметить, что графики на Рис. 14 напоминают графики долготы светового дня $D$ в разные эпохи на Рис. 9, однако области $I$ и $I I$ у них разные. Азимуты точки захода края Солнца, например, в день летнего солнцестояния $A_{G s s}$, как следует из (65), $A_{G s s}=360^{\circ}-A_{G r s m}$. Поэтому графики $A_{G s s m}$ и $A_{G s w n}$ будут похожи на графики долготы светового дня на Рис. 9.

В табл. 3П работы [28] приведены азимуты восходов Солнца для летнего солнцестояния $\left(A_{G r s m}\right)$ и зимнего $\left(A_{G r w n}\right)$ для пяти разных эпох. Изменение широты $\varphi$ дается через $2^{\circ}$, начиная с экватора. Широты изменяются до величин, после которых наступает полярный день $\left(A_{G r s m}=0^{\circ}\right)$ и полярная ночь $\left(A_{\text {Grwn }}=180^{\circ}\right)$.

\section{7. Солнечная тень гномона}

\section{1. Относительная длина тени гномона}

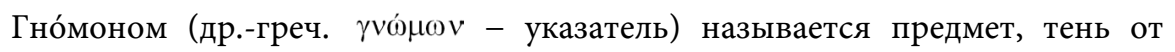


которого используется в солнечных и лунных часах и календарях. На Рис. 15 показана длина тени $l_{s h}$ вертикального гномона длиной $l_{g}$, которая возникает от верхнего края Солнца. Величина $I_{s h}$ зависит от зенитного угла $Z$ Солнца и его углового радиуса $S$. Ранее формулой (21) величина $S$ приводилась в виде дуги $K S_{s 1}$ на Рис. 6. Как следует из Рис. 15, относительная длина тени гномона будет:

$$
l_{\text {sh } 1}=l_{s h} / l_{g}=\operatorname{tg}(z-S)=\operatorname{tg}\left(z-4.6599 \times 10^{-3} / \rho\right),
$$

где зенитный угол $Z$ Солнца определяется выражением (46).

Чтобы избежать отрицательных значений относительной длины теней $I_{s h 1}$ расчет проводится по следующему алгоритму:

$$
\begin{gathered}
\text { Fn06 }=z-4.6599 \times 10^{-3} / \rho ; \\
F n 6=\text { Fn06 при }-0.5 \cdot \pi \leq F n 0 \leq 0.5 \cdot \pi ; \\
F n 6=-0.5 \cdot \pi \text { при } F n 06<-0.5 \cdot \pi ; \\
F n 6=0.5 \cdot \pi \text { при } F n 06>0.5 \cdot \pi ; \\
l_{\text {sh } 10}=\operatorname{tg}(\text { Fn6 }) ; l_{\text {sh } 1}=l_{\text {sh } 10} \text { при } l_{\text {sh } 10}>0 ; l_{\text {sh } 1}=0 \text { при } l_{\text {sh } 10}<0 .
\end{gathered}
$$

\section{2. Изменение длины тени в течение дня}

По алгоритму (69)-(71) рассчитано изменение относительной длины тени $I_{s h 1}$ гномона в течение суток для трех разных дней на широте г. Москвы и для одного дня на широте г. Тюмени (Рис. 16). В левой части графика вертикальными линиями показаны часовые углы восходов Солнца, а в правой-часовые углы его заходов. Так как в эти моменты длина тени стремится к бесконечности, и вертикальный размер графика приходится ограничивать, то асимптотическое приближение линий $l_{s h 1}$ к вертикальным линиям восходов и заходов находится вне рисунка.

Как видно из Рис. 16, в день весеннего равноденствия (линия 1) длина тени $l_{s h 1}$ после восхода Солнца $(\omega<0)$ уменьшается и в полдень $(\omega=0)$ достигает минимального значения $l_{s h l}=1.433$. Затем она увеличивается до бесконечности в момент захода Солнца $(\omega>0)$. В день летнего солнцестояния (линия 2) минимальная длина тени уменьшается до $l_{s h 1}=0.626$, а в день зимнего солнцестояния минимальная длина тени увеличивается до $l_{s h 1}=$ 5.108. Таким образом, на широте г. Москвы длина полуденной тени изменяется в пределах $5.108 \geq 1_{s h 1} \geq 0.626$.

Рассмотрим траекторию движения тени конца гномона в координатах

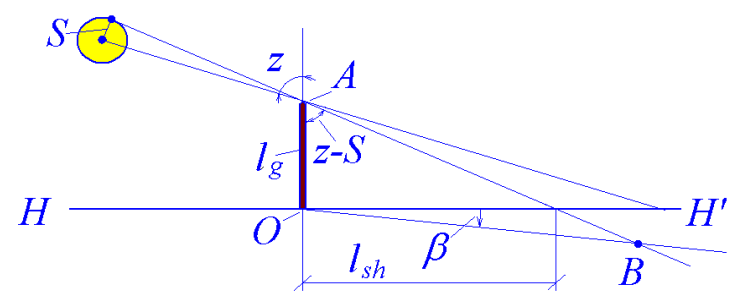

Рис. 15. Длина тени $l_{s h}$ гномона длиной $l_{g}$ при освещении его солнцем $S$. 


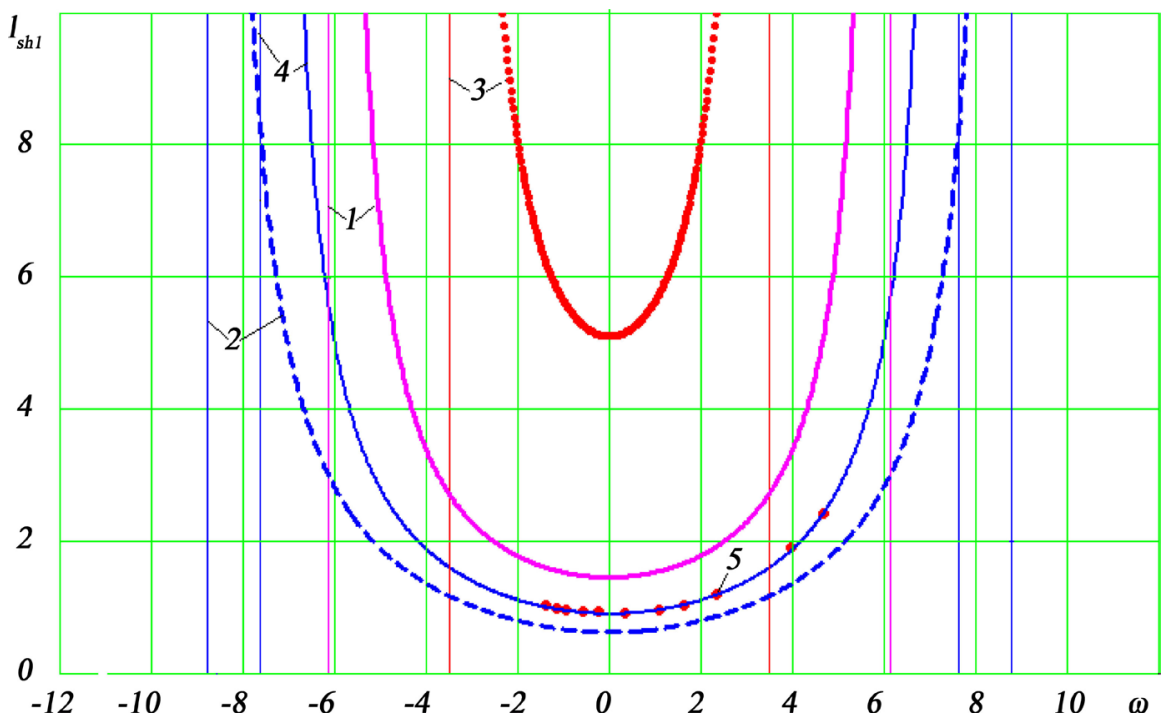

Рис. 16. Изменение длины солнечной тени гномона единичной длины в течение суток в дни весеннего равноденствия 1, летнего 2 и зимнего 3 солнцестояний на широте Москвы $\left(\varphi^{\circ}=55.7522^{\circ}\right)$ в современную эпоху 30.12 .1949 г. 4-длина солнечной тени гномона 15 августа 2015 г. вблизи Тюмени $\left(\varphi^{\circ}=57.301575^{\circ}\right)$ по расчету и 5-по наблюдениям. Вертикальными линиями отмечены часовые углы восходов $(\omega<0)$ и заходов Солнца $(\omega>0)$.

$N_{s h}, E_{s h}$, где ось $N_{s h}$ направлена на Север, а ось $E_{s h}$ - на Восток. Азимут тени $A_{s h}$ отсчитывается от оси $N_{s h}$ и выражается через азимут Солнца так: $A_{s h}=$ $A_{S}+180^{\circ}$. Тогда проекции тени на плоскость горизонта запишутся:

$$
N_{s h}=l_{s h 1} \cdot \cos A_{s h}=-l_{s h 1} \cdot \cos A_{s} ; \quad E_{s h}=-l_{s h 1} \cdot \sin A_{s} .
$$

На Рис. 17 показана траектория дневной тени конца гномона относительной длины для трех дней на широте г. Москвы и одного дня на широте г. Тюмени. В начале координат крестиком отмечено положение гномона. На широте г. Москвы в день весеннего равноденствия (линия 1) конец тени гномона движется с Запада $(\omega<0)$ на Восток почти по прямой линии. При этом тень находится севернее гномона. В день летнего солнцестояния тень гномона (линия 2) начинает утром движение с Юго-запада и заканчивает движение вечером на Юго-востоке. Только вблизи полудня, \pm 2 часа, тень находится севернее гномона. Линией 3 показано, что в день зимнего солнцестояния тень полностью движется на Севере от гномона.

\section{3. Измерение длины тени гномона}

С целью проверки полученных результатов на местности, недалеко от г. Тюмени, были выполнены наблюдения за перемещением тени гномона. На Рис. 18 показан нехитрый инструментарий из подсобных материалов: лист ватмана, компас, карандаш, металлический прут, рулетка и отвес, с помощью которого выполнены наблюдения. Наблюдения проводились 15.08.2015 г., что составляет $T_{d}=148$ день после дня весеннего равноденствия 


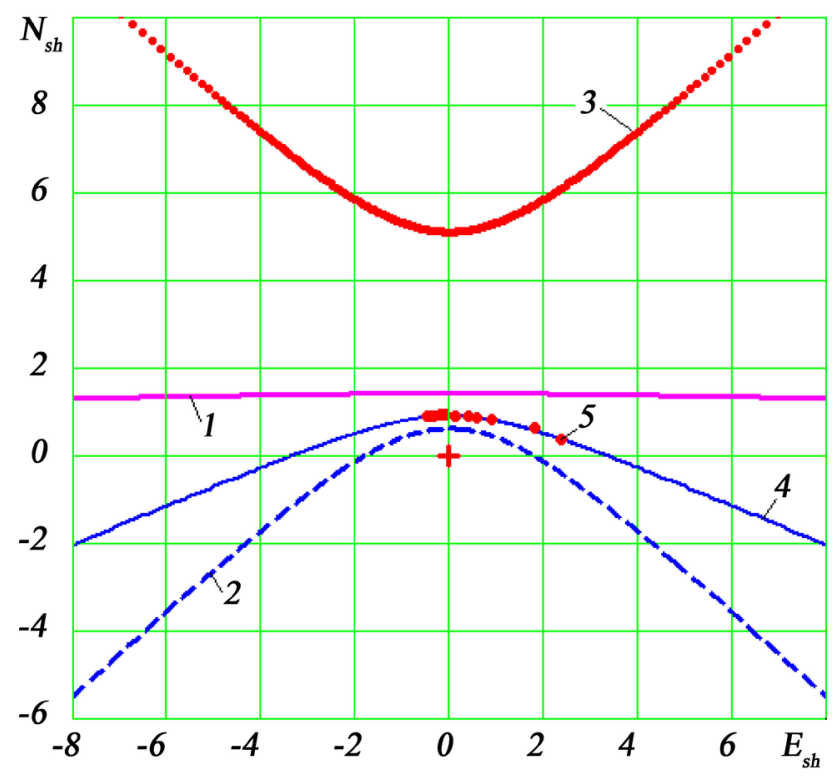

Рис. 17. Суточная траектория тени конца гномона единичной длины на плоскости горизонта $N_{s h}\left(E_{s h}\right)$ в дни весеннего равноденствия 1, летнего 2 и зимнего 3 солнцестояний на широте Москвы $\left(\varphi^{\circ}=55.7522^{\circ}\right)$ в современную эпоху 30.12 .1949 г. 4 -траектория тени гномона 15 августа 2015 г. вблизи Тюмени $\left(\varphi^{\circ}=57.301575^{\circ}\right)$ по расчету и 5-по наблюдениям. Ось $N_{s h}$ направлена на Север, а ось $E_{s h}$-на восток. Положение гномона в начале координат отмечено крестиком.

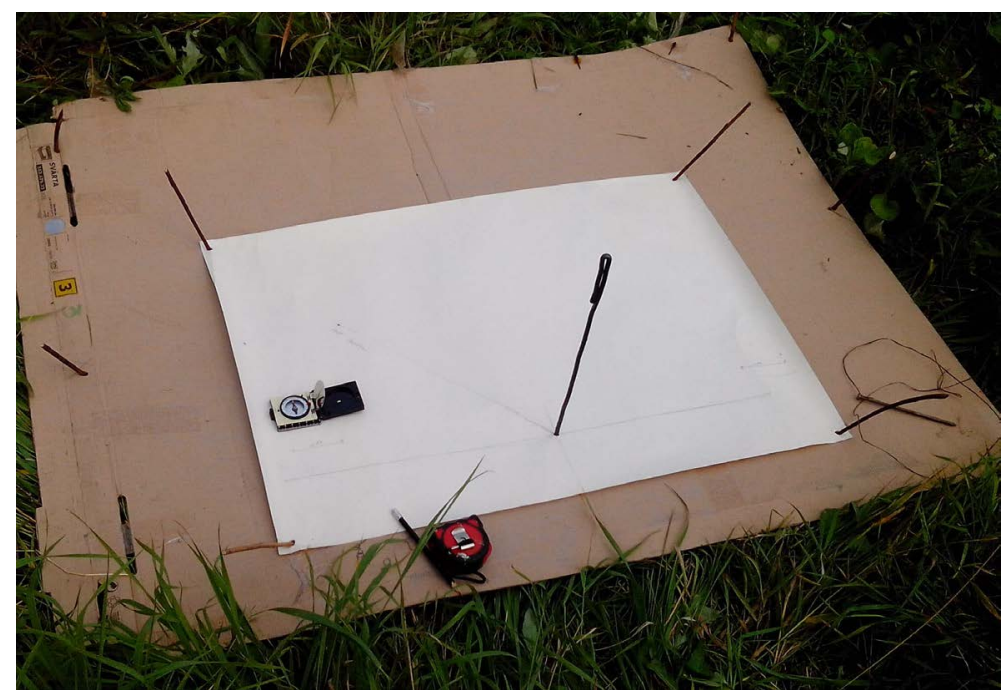

Рис. 18. Наблюдение тени гномона 15 августа 2015 г. вблизи Тюмени $\left(\varphi^{\circ}=\right.$ $57.301575^{\circ}$ ) и основные инструменты: лист ватмана, компас, карандаш, металлический прут диаметром 6 мм-гномон, рулетка и отвес.

21 марта. Вдоль тени карандашом проводились линии на ватмане и отмечались конец тени и время ее наблюдения. Затем были замерены азимуты $A_{s h M}$ теней от направления от направления на Север $N_{M}$ определенный по компасу.

На Рис. 19 показаны результаты наблюдений в виде зависимости относительной длины тени $l_{s h l}$ от времени в г. Тюмени $t_{m}$. Как видно, 


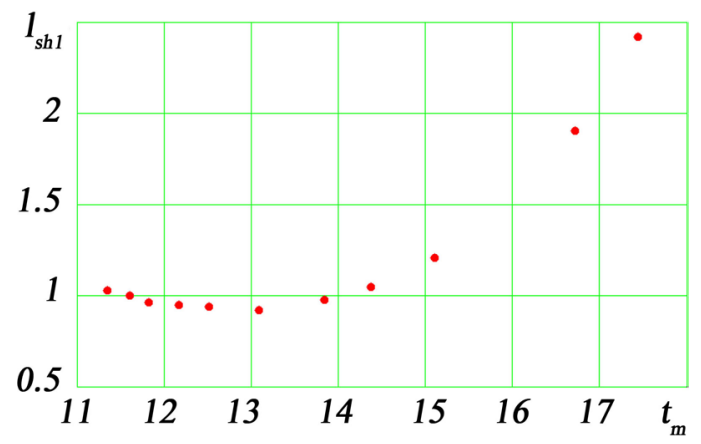

Рис. 19. Результаты наблюдения относительной длины тени $l_{s h 1}$ гномона в зависимости от времени $t_{m}$ в г. Тюмени 15 августа 2015 г. на широте $\varphi^{\circ}=57.301575^{\circ}$.

минимум $l_{s h 1}$ приходится на время $t_{m}$ отличное от 12 часов. На Рис. 16 данные наблюдения точками 5 нанесены на рассчитанную согласно (69)-(71) зависимость $4 l_{s h 1}(\omega)$. Положение полдня $(\omega=0)$ приходится на 12 ч. 44 мин тюменского времени.

Для рассматриваемой широты места наблюдения и дня $T_{d}=148$ по формуле (50) был рассчитан азимуты $A_{S}$ Солнца. Аналогично Рис. 10 он был представлены в виде графика $A_{S}(\omega)$. В этих же координатах нанесены определенные азимуты Солнца $A_{S M}$ по замеренным азимутам тени $A_{S M}=$ $A_{s h M}-180^{\circ}$. Азимуты $A_{S M}$ оказались эквидистантно сдвинуты на $\Delta A=$ $14.47059^{\circ}$ вниз по отношению к азимутам $A_{S}$ т.е. $A_{S M}<A_{S}$. Это обусловлено тем, что Северный магнитный полюс сдвинут к Востоку на величину $\triangle A$ по отношению к географическому Северному полюсу.

Скорректированные данные измерения азимута тени и времени ее наблюдения использованы для расчета по формулам (72) ее траектории. На Рис. 17 она нанесена точками 5. Как видим, измеренная траектория тени гномона 5 совпала с рассчитанной 4. Некоторый разброс точек 5 вокруг линии 4 объясняется погрешностью измерений.

Итак, выполненные наблюдения и измерения тени гномона подтвердили представленный алгоритм её расчета. Кроме того, измерения позволили определить истинный полдень в 12 ч. 44 мин тюменского времени и восточное отклонение $\Delta A$ магнитной стрелки. Следует отметить, что азимут солнечного полдня и его время наступления можно непосредственно определить по данным наблюдения, представленным на Рис. 19. Но для этого наблюдения необходимо производить более часто и за больший промежуток времени.

\section{4. Полуденная длина тени гномона в течение года}

Как видно из Рис. 16, наименьшая тень наступает в полдень $(\omega=0)$. В этом случае зенитный угол $Z_{n}$ центра Солнца определяется выражением (51), тогда в соответствии с (68) единичная длина полуденной тени запишется так

$$
l_{s 1 n}=\operatorname{tg}\left(\varphi-\delta-4.6599 \times 10^{-3} / \rho\right) .
$$


Алгоритм расчета относительной длины тени в п. 17.3 программы на SunPhnmen.mcd приведен с учетом особенностей функции tg. Результаты расчетов изменения длины полуденной тени в течение года на разных широтах Северного полушария показаны на Рис. 20. В этих расчетах ввиду несущественного влияния относительного расстояния $\rho$ принято $\rho=1$. Как видно из Рис. 20 , на широте $\varphi=60^{\circ}$ в день весеннего равноденствия $\left(T_{d}=0\right)$ длина относительной полуденной тени $l_{s 1 n}=1.687$. Затем она уменьшается и в день летнего солнцестояния достигает минимального значения 0.734 . Далее увеличивается и принимает максимальное значение 8.351 в день зимнего солнцестояния. С увеличением широты $\varphi$ минимальные и максимальные значения возрастают, а с уменьшением $\varphi$ - уменьшаются. В тропических широтах $(\varphi<\varepsilon)$ зависимость $1_{s 1 n}(\varphi)$ имеет два максимума и два минимума. В минимумах длина тени равна нулю: в эти моменты Солнце находится в зените. А максимумы $l_{s 1 n}$ наступают в дни солнцестояний.

\section{5. Экстремальные длины солнечной тени гномона в дни солнцестояний}

Как показано выше, экстремальные длины теней происходят в дни солнцестояний, которые характеризуются углом $\delta= \pm \varepsilon$. Тогда из (73) с учетом $\rho=1$ получаем относительные длины теней

$$
l_{s 1 s}=\operatorname{tg}\left(\varphi-\varepsilon-4.6599 \times 10^{-3}\right) ; l_{s 1 w}=\operatorname{tg}\left(\varphi+\varepsilon-4.6599 \times 10^{-3}\right),
$$

для дней летнего и зимнего солнцестояний, соответственно. С учетом особенностей функции $\mathrm{tg}$ алгоритм расчета приведен в п. 17.3 программы на SunPhnmen.mcd.

Результаты расчетов экстремальных длин тени для пяти разных эпох в зависимости от широты $\varphi$ даны на Рис. 21. Шкала для длин теней в летнее солнцестояние $l_{s 1 s}$ приведена справа, а для зимнего солнцестояния $l_{s 1 \text { w }}$

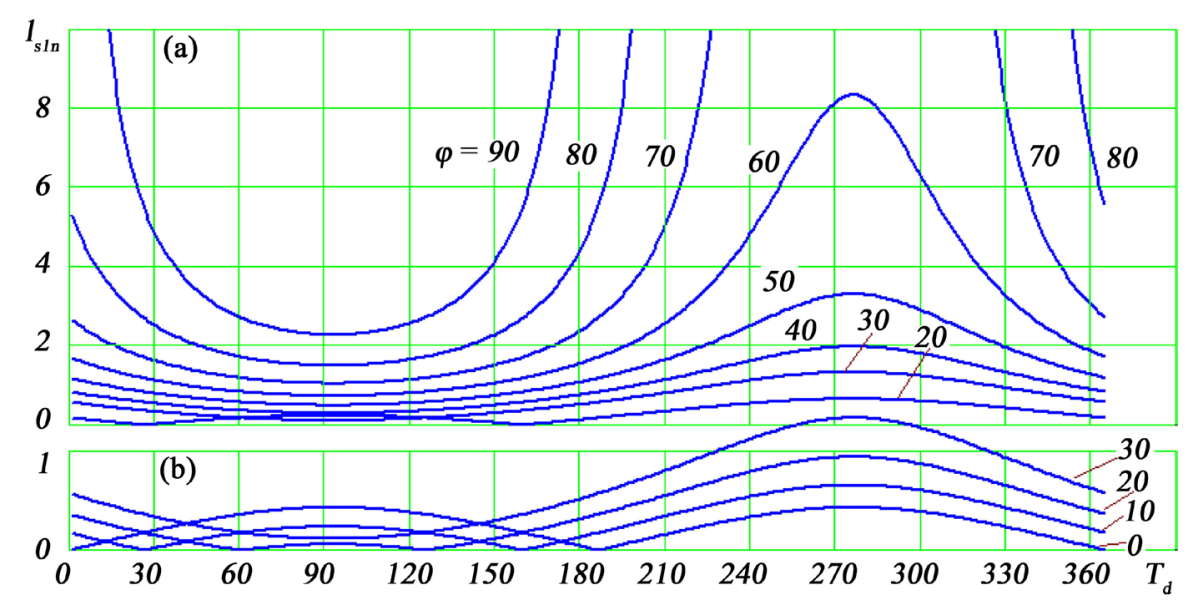

Рис. 20. Длина полуденной солнечной тени гномона единичной длины $l_{s 1 n}$ в зависимости от дней года $T_{d}$ в современную эпоху 30.12.1949 г. на разных широтах $\varphi^{\circ}$ Северного полушария: (a) в обычном масштабе; $(b)$-при увеличении ординаты $l_{s 1 n}$ в 2 раза. 


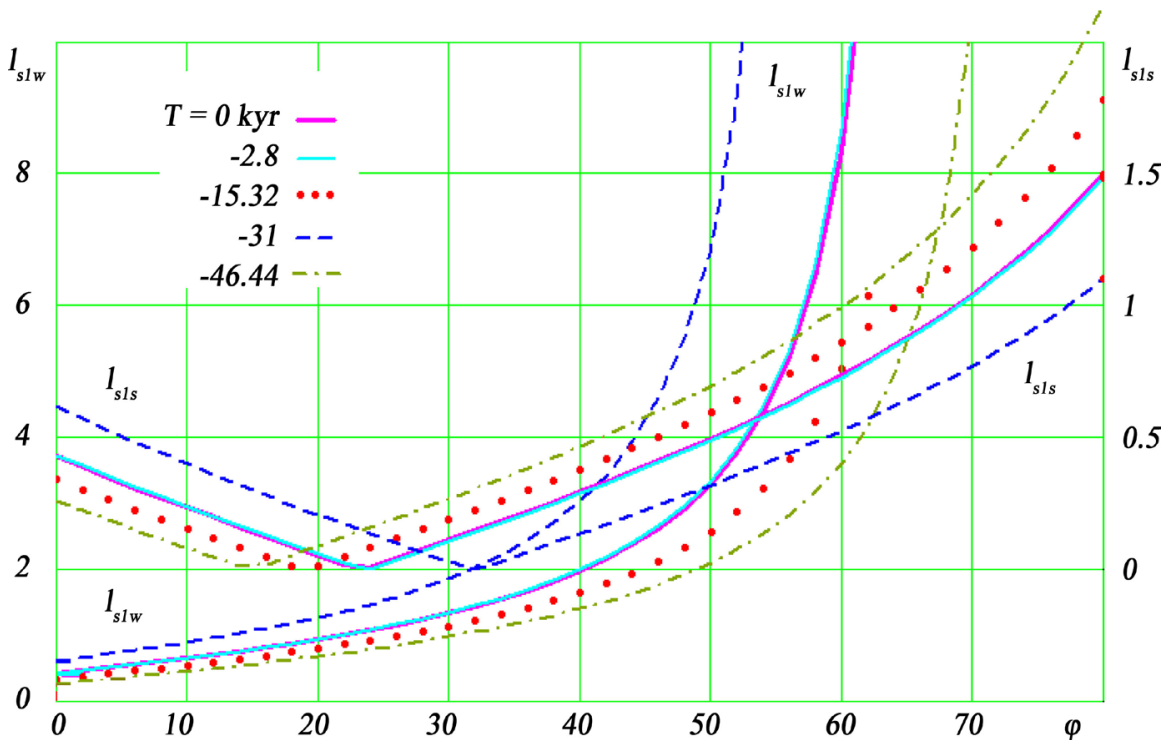

Рис. 21. Тени гномона единичной длины в полдни летнего солнцестояния $\left(l_{s 1 s}\right)$ и зимнего солнцестояния $\left(l_{s 1 w}\right)$ на разных широтах $\varphi^{\circ}$ Северного полушария в экстремальные эпохи $T$ за последние 50 т.л.н.: $T$-время в тыс. лет от 30.12.1949 г.; масштабы длин $l_{s l \text { w }}$ и $l_{s l s}$-разные и отличаются в 4 раза.

слева. В современную эпоху, как видно из Рис. 21 при $T=0$, в полдень летнего солнцестояния на экваторе $(\varphi=0)$ длина тени $I_{s 1 s}=0.428$. Затем она уменьшается на тропике $\varphi \approx \varepsilon$ до 0 , а с дальнейшим увеличением широты длина тени возрастает до $I_{s 1 s}=2.277$ на широте $\varphi=90^{\circ}$.

В другие эпохи графики полуденной тени в летнее солнцестояние почти эквидистантно сдвигаются по широте $\varphi$, в зависимости от угла наклона $\varepsilon$. На широтах $\varphi=\varepsilon$ длина тени равна нулю: $I_{s 1 s}=0$.

В современную эпоху $(T=0)$ в полдень зимнего солнцестояния на экваторе $(\varphi=0)$ длина тени $I_{s l w}=0.428$, т.е. такая же, как и в полдень летнего солнцестояния. Затем она с увеличением широты $\varphi$ растет до бесконечности до широты $\varphi=90^{\circ}-\varepsilon^{\circ}$ полярного круга, где начинается полярная ночь. В другие эпохи полуденная тень на экваторе изменяется от 0.259 ( $T=46.44$ т.л.н.) до 0.618 ( $T=31$ т.л.н.). С увеличением широты длины теней в день зимнего солнцестояния увеличиваются во все эпохи и тем в большей степени, чем меньше широта полярного круга $\left(90^{\circ}-\mathcal{\varepsilon}^{\circ}\right)$.

В табл. 4П работы [28] приведены длины полуденных теней в дни летнего $\left(l_{s l s}\right)$ и зимнего $\left(l_{s l w}\right)$ солнцестояний для пяти разных эпох. Изменение широты $\varphi$ дается через $2^{\circ}$, начиная с экватора до широты $80^{\circ}$. Бесконечная длина тени выражена числом $1.6 \mathrm{e}+16$.

\section{8. Основные феномены Солнца}

Подведем итоги по основным феноменам Солнца. Освещение Солнцем поверхности в точке нахождения наблюдателя $M$ (см. Рис. 2), т.е. ее инсоляция, зависит от эксцентриситета $е$ орбиты Земли, угла наклона $\varepsilon$ плоскости экватора Земли к плоскости ее орбиты, угла $\varphi_{p \gamma}$ между 
перигелием орбиты Земли и восходящим углом $\gamma$ и широты места $\varphi$ [19]. Эти четыре параметра создают разнообразие инсоляции поверхности Земли, из которого наблюдатель может определить следующие основные 6 явлений, или феноменов (см. Таблица 3). Первым феноменом является количество дней до начала сезона $T_{d}$ отсчитываемое от момента весеннего равноденствия. Для весны $T_{d s p}=0$, для лета наблюдатель определяет количество дней до летнего солнцестояния $T_{d s m}$, для осени-до момента осеннего равноденствия $T_{d a u}$ и для зимы-до момента зимнего солнцестояния $T_{d w n}$. С этим феноменом также связана продолжительность сезонов $\Delta T_{d}$ которая, согласно (6) рассчитывается по разности величин $T_{d}$. В рассмотренном выше алгоритме начала сезонов определяются долготами $\lambda=0 ; \pi / 2 ; \pi ; 3 \pi / 2$. По зависимости $\lambda\left(T_{d}\right)$, определяется время $T_{d}$ в днях до начала каждого сезона. Этот феномен не зависит от широты $\varphi$ наблюдателя, а определяется только параметрами $e, \delta$ и $\varphi_{p r}$

Второй феномен, характерный для высоких широт, 一это длительность в днях полярных дней $\left(\Delta T_{d d}\right)$ и полярных ночей $\left(\Delta T_{d n}\right)$. Наблюдатель на широте $\varphi$ своего места может определить длительность полярных ночи и дня. А в теории полярные ночи и дни определяются долготами $\lambda_{p d}$ и $\lambda_{p n}$ (см. формулы (35)-(36)), по которым длительность этих феноменов рассчитывается по зависимости $\lambda\left(T_{d}\right)$. Этот феномен сильно зависит от широты $\varphi$ наблюдателя. Поэтому он в наибольшей степени находит для уточнения широты палеонаблюдателя, если он находился в высоких широтах.

Третьим феноменом является зенитный угол $Z_{n}$ Солнца в полдни равноденствий и солнцестояний. Как уже отмечалось для весны, лета, осени и зимы Северного полушария равны, соответственно:

$$
z_{n s p}=\varphi, \quad Z_{n s m}=\varphi-\varepsilon, \quad z_{\text {nаu }}=\varphi \text { и } \quad Z_{n w n}=\varphi+\varepsilon .
$$

Таблица 3. Основные феномены Солнца.

\begin{tabular}{|c|c|c|c|c|c|c|}
\hline \multirow{2}{*}{$\begin{array}{c}\text { № } \\
\text { П/ח }\end{array}$} & \multirow[b]{2}{*}{ Наименование } & \multirow[b]{2}{*}{ Параметр } & \multicolumn{4}{|c|}{ Сезоны и долготы $\lambda$ их начала } \\
\hline & & & Весна 0 & Лето $\pi / 2$ & Осень $\pi$ & $\begin{array}{c}\text { Зима } \\
3 \pi / 2\end{array}$ \\
\hline 1 & Количество дней до начала сезона & $T_{d}$ дни & 0 & $T_{d s m}$ & $T_{d a u}$ & $T_{d w n}$ \\
\hline 2 & Длительность полярных дней и ночей & $\Delta T_{d d, n}$, дни & - & $\Delta T_{d d}$ & - & $\Delta T_{d n}$ \\
\hline 3 & $\begin{array}{l}\text { Зенитные углы Солнца в полдни } \\
\text { равноденствий и солнцестояний }\end{array}$ & $z_{n}$ & $Z_{n s p}$ & $z_{n s m}$ & $Z_{\text {nau }}$ & $Z_{n w n}$ \\
\hline 4 & $\begin{array}{l}\text { Долгота светового дня в дни } \\
\text { солнцестояний }\end{array}$ & $D$, часы & - & $D_{s}$ & - & $D_{w}$ \\
\hline 5 & $\begin{array}{l}\text { Азимуты восхода Солнца в дни } \\
\text { равноденствий и солнцестояний }\end{array}$ & $A_{G r}$ & $A_{\text {Grsp }}$ & $A_{G r s m}$ & $A_{\text {Grau }}$ & $A_{G r w n}$ \\
\hline 6 & $\begin{array}{l}\text { Относительные длины теней гномона } \\
\text { в полдни равноденствий и } \\
\text { солнцестояний }\end{array}$ & $l_{s 1 n}$ & $1_{s l s p}$ & $l_{s l s}$ & $1_{\text {slau }}$ & $I_{s 1 W}$ \\
\hline
\end{tabular}


Следует отметить, что в наблюдениях может использоваться высота 1 Солнца над горизонтом. Она определяется через зенитный угол так:

$$
l=\pi / 2-z_{n} .
$$

Четвертый феномен-это долгота светового дня во время летнего солнцестояния $D_{s}$ и зимнего солнцестояния $D_{w^{*}}$ Для наблюдателя существует сложность в измерении этого феномена. Эти измерения требуют высокой технологии определения времени и хранения сведений о нем. Тем не менее, возможны приемы выполнения этой работы. В теории этот феномен определяется выражением (43).

Пятый феномен-азимуты восходов и заходов Солнца в дни солнцестояний и равноденствий. Они могут быть зафиксированы наблюдателем в зависимости от какого-то направления. В теории азимут восхода Солнца в день летнего солнцестояния определяется выражениями (66), а зимнего-(67). В дни равноденствий при $\delta=0$, согласно (60) и (64) азимут восхода Солнца будет:

$$
A_{\text {Grsp }}=A_{\text {Grau }}=90^{\circ} \cdot\left[1-\left(9.8902 \times 10^{-3}+4.6599 \times 10^{-3}\right) \cdot \operatorname{tg} \varphi / 0.5 \pi\right] .
$$

Шестой феномен-длина теней гномона в полдни. Для дней солнцестояний: летнего $\left(l_{s 1 s}\right)$ и зимнего $\left(l_{s 1 w}\right)$ определяется согласно (74), а в дни равноденствий при $\delta=0$, согласно (73), длина тени будет:

$$
l_{\text {s1sp }}=l_{\text {s1au }}=\operatorname{tg}\left(\varphi-4.6599 \times 10^{-3}\right) .
$$

Наблюдатель может зафиксировать эти феномены по отношению к гномону, длина которого неизвестна. В этом случае могут потребоваться дополнительные данные для определения относительной длины тени. В теории относительные длины теней определяются выражениями (74) и (78).

\section{9. Восстановление параметров наблюдателя и движения Земли по древним феноменам Солнца}

Параметрами наблюдателя являются его древние широта местонахождения и направление на Север. Исследователи древних календарей отмечают изменение этих параметров наблюдателя. Например, М.И. Исрапилов пришел к выводу о смещении Северного полюса в Якутию несколько тысяч лет назад [14]. Рассмотрим как определить параметры наблюдателя по феноменам Солнца.

В дни равноденствий зенитный угол $Z$, согласно (75), в полдень будет равняться широте места $Z_{n s p}=\varphi$. А длина тени $I_{s 1 s p}$ согласно (77) также зависит от широты $\varphi$. Тогда широта места наблюдателя определяется по одному из этих феноменов:

$$
\varphi=z_{\text {nsp }} \text { либо } \varphi=\operatorname{arctg}\left(l_{\text {s1sp }}+4.6599 \times 10^{-3}\right) .
$$

В дни равноденствий суточное движение Солнца происходит по кругу 
экватора $A A^{\prime}$ (Рис. 2). Поэтому центр Солнца восходит на Востоке, а заходит на Западе. Палеонаблюдатель отмечает восходы и заходы Солнца по его верхнему краю. По формуле (77) при известной широте $\varphi$ может быть найден азимут $A_{G r s p}$ восхода верхнего края Солнца. Обозначим наблюдаемое значение азимута палео-Востока как $A_{E a p a}=90^{\circ}$, тогда из (77) можем записать:

$$
A_{\text {Eapa }}=A_{G 1 s p}+90^{\circ} \cdot\left(9.8902 \times 10^{-3}+4.6599 \times 10^{-3}\right) \cdot \operatorname{tg} \varphi / 0.5 \pi .
$$

Тогда азимут палео-Севера запишется так:

$$
\begin{aligned}
A_{\text {Npa }} & =A_{\text {Eapa }}-90^{\circ} \\
& =A_{G 1 \text { spa }}-90^{\circ} \cdot\left[1-\left(9.8902 \times 10^{-3}+4.6599 \times 10^{-3}\right) \cdot \operatorname{tg} \varphi / 0.5 \pi\right]
\end{aligned}
$$

где $A_{G 1 s p a}$ - наблюдаемый азимут древнего восхода Солнца во время весеннего равноденствия.

Азимут палео-Севера также можно определить через азимут восхода Солнца в момент осеннего равноденствия, а также через азимуты заходов в дни равноденствий. Четырехкратные способы определения предоставляют возможности для проверки результатов определения $A_{N p a}$.

Теперь на основе феноменов Солнца определим параметры движения Земли. В полдень солнцестояний $(\lambda=\pi / 2,1.5 \pi)$ зенитные углы $z$ Солнца, согласно (75), и длины тенней в полдень, согласно (74), зависят от $\varphi-\varepsilon$ и $\varphi$ $+\varepsilon$. Поэтому при известной широте $\varphi$ угол наклон $\varepsilon$ плоскости экватора к плоскости орбиты Земли может быть определен по одной из четырех зависимостей:

$$
\begin{gathered}
\varepsilon=\varphi-z_{n s m} ; \quad \varepsilon=-\left(\varphi-z_{n s m}\right) ; \varepsilon=\varphi-\operatorname{arctg}\left(l_{s 1 s m}\right)-4.66 \times 10^{-3} ; \\
\varepsilon=-\left[\varphi-\operatorname{arctg}\left(l_{s 1 w n}\right)\right]-4.66 \times 10^{-3} .
\end{gathered}
$$

Как видим, зависимости (82) позволяют разными четырьмя способами определить угол наклона $\mathcal{E}$ при известной широте $\varphi$. Если широта $\varphi$ неизвестна, то соотношения (82) позволяют определить и широту $\varphi$ и угол $\mathcal{\varepsilon}$, при этом двумя способами.

Предельные азимуты восходов в дни солнцестояний определяются выражениями (66) и (67), которые зависят от $\mathcal{E}$ и $\varphi$. Вычтем из (67) выражение (66) и выразим угол наклона $\varepsilon$.

$$
\varepsilon=\arcsin \left[\sin \left(\pi \cdot \Delta A_{s r} / 360\right) \cdot \cos \varphi\right]
$$

где $\Delta A_{s r}=A_{G r w n}-A_{G r s m}-$ разница азимутов восходов в дни солнцестояний.

Аналогичное выражение для угла наклона запишется через разницу $\Delta A_{s s}$ азимутов заходов в дни солнцестояний. Поэтому, если величины $\Delta A_{s s}$ и $\Delta A_{s r}$ имеются, то этим предоставляется возможность проверки определения $\varepsilon$. Выражение (83) знаменательно тем, что в него входит разность азимутов восходов. Это исключает возможное, отличное от современного, толкование восхода и определение его момента. В этом случае на 
определение $\varepsilon$ также меньше влияют географические особенности места наблюдения.

Долготы светового дня в моменты солнцестояний определяются выражениями (43). С учетом (37)-(40) найдем разность светового дня $\Delta D=$ $D_{s}-D_{w}$

$$
\Delta D=\frac{24}{\pi}\left(\arccos \left(\frac{\cos z_{0 a 1}-\sin \varepsilon \cdot \sin \varphi}{\cos \varepsilon \cdot \cos \varphi}\right)-\arccos \left(\frac{\cos z_{0 a 1}-\sin \varepsilon \cdot \sin \varphi}{\cos \varepsilon \cdot \cos \varphi}\right)\right) \text {. }
$$

Упростим выражение (84), рассматривая его для центра Солнца, т.е. согласно (23) $z_{0 a 1}=\pi / 2$. Тогда оно приобретет вид:

$$
\Delta D=\frac{24}{\pi}[\arccos (-\operatorname{tg} \varphi \cdot \operatorname{tg} \varepsilon)-\arccos (\operatorname{tg} \varphi \cdot \operatorname{tg} \varepsilon)],
$$

После упрощения (85) выразим угол наклона $\varepsilon$ :

$$
\varepsilon=\operatorname{arctg}\left\{\left[\sin \left(0.5 \pi \cdot \Delta D_{u d}\right)\right] / \operatorname{tg} \varphi\right\},
$$

где

$$
\Delta D_{u d}=\Delta D / 24
$$

- разность светового дня в моменты солнцестояний, по отношению к длительности суток.

Выражение (86) позволяет определить угол наклона $\mathcal{\varepsilon}$ при известной широте места $\varphi$. Оно не требует знания способа деления суток на части, так как в (86) входит доля от суток, на которую солнечный день в летнее солнцестояние больше дня в зимнее солнцестояние. После нахождения $\varepsilon$ из (86), по выражению (84) можно уточнить $\Delta D$, а затем по (86) определить уточненное значение угла наклона $\mathcal{\varepsilon}$.

Определенные по представленным формулам широта $\varphi$ и угол наклона $\varepsilon$ в дни равноденствий и солнцестояний содержат ошибки, связанные с определением палеонаблюдателем азимута, длины тени, продолжительности разности дня $\Delta D$. Кроме того в формулах содержится ошибка, связанная с тем, что принятые моменты равноденствий и солнцестояний на заходе и восходе Солнца или в полдень могут не совпадать с моментом прохождения Солнца через соответствующие точки $\gamma, \gamma^{\prime}, E$ и $E^{\prime}$ на эклиптике $E E^{\prime}$ (Рис. 2). Разность моментов может достигать 0.5 дня. Он дает погрешность пропорциональной отношению 0.5 дня к периоду прецессии оси Земли, равному 25,780 тыс. лет. То есть, эта погрешность несущественна. Погрешность наблюдений палеонаблюдателя также может быть оценена. Для этого необходимо современному наблюдателю повторить наблюдения в месте нахождения палеонаблюдателя. Обработка их результатов позволит определить погрешность палеонаблюдателя.

При известных палеопараметрах $\varphi$ и $\mathcal{\varepsilon}$ остальные параметры: эксцентриситет $e$ орбиты Земли и долгота $\varphi_{p \gamma}$ перигелия могут быть определены по количеству дней $T_{d s m}, T_{d a w}, T_{d w n}$ до начала сезона и по длительности полярных дней $\Delta T_{d d}$ или ночей $\Delta T_{d n}$. Эти параметры зависят от долготы $\lambda$, 
которая рассчитывается численно в прилагаемой программе на SunPhnmen.mcd. Методом последовательных приближений с ее помощью параметры $e$ и $\varphi_{p \gamma}$ могут быть найдены. Они могут быть определены разными способами, так как для нахождения двух неизвестных $e$ и $\varphi_{p \gamma}$ существует 5 уравнений для величин $T_{d s m}, T_{d a u}, T_{d w n}, \Delta T_{d d}$ и $\Delta T_{d n}$.

Изменение параметров $\varepsilon$, $e$ и $\varphi_{p \gamma}$ во времени известно в результате решения задач об орбитальном движении Земли и о ее вращательном движении. В файле OrAl-5kyr.prn величины $\varepsilon$, $е$ и $\varphi_{p \gamma}$ даны с интервалом 1 год за 5 т.л.н., а в файле OrAl-200ky.prn - с интервалом 40 лет за 200 т.л.н. Поэтому, по трем параметрам $\varepsilon$, $е$ и $\varphi_{p \gamma}$ должен однозначно определиться возраст эпохи палеонаблюдателя. Если из археологических исследований примерный возраст эпохи наблюдения известен, то по одной из зависимостей $\mathcal{E}$, е или $\varphi_{p \gamma}$ от времени этот возраст может быть уточнен.

Рассмотрим схематически алгоритмы определения параметров $е$ и $\varphi_{p \gamma}$ в двух случаях: 1) при известном примерном возрасте эпохи наблюдения и 2) при неизвестном. Пусть и в первом и во втором случае определены два каких-либо параметра палеоклимата, например, $T_{d s m, p}$ и $\Delta T_{d d, p}$. Индекс $p$, здесь и далее добавленный к обозначению параметра, обозначает, что этот параметр определен по древнему (палео-) календарю.

В первом случае пусть $T_{1}$ и $T_{2}$-время начальной и конечной эпох, в пределах которого находится предполагаемый возраст древнего календаря. С помощью программы на SunPhnmen.mcd на интервале от $T_{1}$ до $T_{2}$ рассчитываются $T_{d s m}$ и $\Delta T_{d d}$ и строятся графики $T_{d s m}\left(\varphi_{p \gamma}\right)$ и $\Delta T_{d d}(e)$. По этим графикам по величинам $T_{d s m, p}$ и $\Delta T_{d d, p}$ определяются угол перигелия $\varphi_{p \gamma, p}$ и эксцентриситет $e_{p}$, которые были в эпоху древнего календаря. Время эпохи $T_{p}$ определяется из файла данных OrAl-5kyr.prn или OrAl-200ky.prn.

Во втором случае, когда возраст календаря неизвестен, при заданных параметрах $\varphi$ и $\mathcal{E}$ с помощью программы на SunPhnmen.mcd производится первая серия расчетов при неизменном угле перигелия $\varphi_{p \gamma}$ варьируется эксцентриситет $e$. Во второй серии при неизменном эксцентриситете $e$ варьируется угол перигелия $\varphi_{p r}$ Затем строится первая серия графиков $T_{d s m}\left(\varphi_{p \gamma}\right)$ при разных $е$ и вторая серия графиков $\Delta T_{d d}(e)$ при разных $\varphi_{p r}$ Затем на графиках $T_{d s m}\left(\varphi_{p \gamma}\right)$ проводится горизонтальная линия $T_{d s m, p}=$ const, а на графиках $\Delta T_{d d}(e)-\Delta T_{d d, p}=$ const. По пересечению этих линий с линиями графиков $T_{d s m}\left(\varphi_{p \gamma}\right)$ и $\Delta T_{d d}(e)$ определяются параметры: угол $\varphi_{p \gamma, p}$ и эксцентриситет $e_{p}$, которые были в эпоху древнего календаря. Время эпохи $T_{p}$ определяется из файла данных OrAl-5kyr.prn или OrAl-200ky.prn по трем параметрам: $\mathcal{E}, \varphi_{p \gamma, p}$ и $e_{p}$.

\section{0. Использование результатов в аэрокосмических исследованиях рельефа}

Рассмотренный алгоритм и программа на SunPhnmen.mcd по расчету феноменов Солнца может быть использована для определения высоты 
возвышенностей и глубины выемок при аэрокосмическом исследовании поверхности Земли или других планет. В качестве примера рассмотрим определение профиля бугра по его тени.

Осенью 2013 г. оленеводами на Ямале, в 30 км Бованенковского месторождения (70²1'44" с.ш. и 68²6'46" в.д.) [29] обнаружен провал в земле, который еще называют воронкой. Он имел диаметр 25 м и глубину больше 50 м [30]. Вверху провал завершается коническим раструбом диаметром около 60 м. По космическому снимку от 09.06.2013 г. (см. Рис. 22(a)) было установлено [30], что на месте провала находился бугор 1. Цифрой 2 показана тень от бугра.

Солнце находится в направлении противоположном тени, и, как следует из Рис. 22(а), азимут Солнца равен $A_{S h l}=326.5^{\circ}$. Широта бугра $\varphi=70.3622^{\circ}$, а дата его наблюдения соответствует $T_{d}=81$ день от дня равноденствия 21 марта. По зависимости азимута Солнца $A_{s}$ от часового угла $\omega(50)$ время $T_{d}$ и широта бугра $\varphi$ позволяют рассчитать часовой угол Солнца $\omega$, который обозначим как $\omega_{h s}$. Эти расчеты выполнены в в п. 18 программы на SunPhnmen.mcd.

Ряд азимутов Солнца $A_{S, k}$ по формуле (50) рассчитан для ряда часовых углов $\omega_{h, k}$ По значению азимута $A_{S h l}$ из ряда азимутов $A_{S, k}$ выбирается индекс in 12 , при котором азимут $A_{S, i n 12}$ находится вблизи значения $A_{S h}$, но меньше его. Тогда часовой угол Солнца находится интерполяцией по следующей формуле.

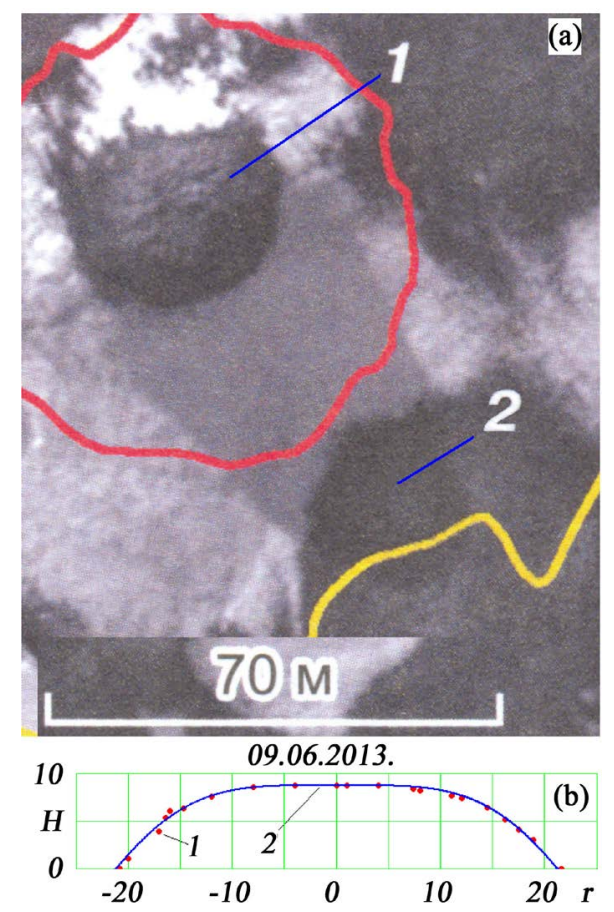

Рис. 22. Бугор (а) от 9 июня 2013 г. вблизи Бованенково на Ямале [30] перед появлением осенью 2013 г. воронки и профиль бугра в метрах (b), рассчитанный по его тени: точки-по результатам измерения тени, линия-апроксимационная зависимость (90). 


$$
\omega_{h S}=\omega_{h, i n 12}+\frac{\left(\omega_{h, i n 12+1}-\omega_{h, i n 12}\right)\left(A_{S, i n 12+1}-A_{S h l}\right)}{A_{S, i n 12+1}-A_{S, i n 12}} .
$$

По часовому углу Солнца $\omega_{h s}$ с помощью формулы (71) рассчитывается единичная длина гномона $l_{s h l h l}$ в месте нахождения бугра. Так как формула (71) и входящие в нее выражения являются сложными нелинейными зависимостями, то вычисления в п. 18 программы на SunPhnmen.mcd производятся аналогично определению часового угла по формуле (88).

Наблюдаемую длину тени бугра $l_{O s h}$ можно определить по космоснимку (Рис. 22(а)) с учетом приведенного на нем масштаба длины, равному 70 м (см. Рис. 22). Тогда высота бугра определяется так:

$$
H=\frac{l_{\text {Osh }}}{l_{\text {sh1hl }}} .
$$

Такие расчеты выполнены для ряда точек тени и получен профиль бугра. На Рис. 22(b) точками 1 представлен профиль бугра, определенный по замерам длины тени. Линией 2 приведена аппроксимация профиля бугра зависимостью

$$
H_{c s}=H_{m x} \cdot \cos \left[0.5 \pi\left(\frac{r}{R_{h}}\right)^{2}\right]
$$

где $H_{m x}=8.76$ м - высота бугра в центральной точке;

$R_{h}=21.2$ м - радиус бугра.

В работе [30] по стереопаре снимков оценили диаметр бугра в основании: 45 - 48 м и его высоту: 5 - 6 м. Как видим, полученные по тени размеры бугра согласуются с этими оценками: диаметр бугра по тени меньше на 9.7\%, а высота - больше на 37\%. В работе [30] сообщается, что в месте нахождения воронки наблюдается уклон местности. Возможно, наличие уклона увеличивает высоту холма.

Рассчитаем тень от гномона, когда она падает не на горизонтальную поверхность, а имеющую уклон $\beta$ к горизонтальной поверхности $H^{\prime}$ (см. Рис. 15). Обозначим длину тени гномона на наклонной поверхности $1_{s h \beta}=$ $O B$. В треугольнике $A O B$ угол $B=90^{\circ}-(z-S)-\beta$. Тогда по теореме синусов $l_{g} / \sin B=O B / \sin (z-S)$ находим длину тени гномона на наклонной поверхности $O B$

$$
l_{\text {sh } \beta}=l_{g} \cdot \frac{\sin (z-S)}{\cos (z-S+\beta)} .
$$

После преобразования (91) длину тени гномона единичной длины на поверхности с углом уклона $\beta$ получаем в виде:

$$
l_{\text {sh } 1 \beta}=\frac{l_{\text {sh1 }}}{\cos \beta-l_{s h 1} \cdot \sin \beta},
$$

где $l_{s h 1}$-длина тени гномона единичной длины на горизонтальной поверхности; 
$\beta$-угол наклона поверхности в направлении тени.

При наличии уклона в месте нахождения бугра длина тени согласно (92) увеличится. Это приведет, в соответствии с (89), к уменьшению высоты $H$ бугра.

Представляет интерес полученный профиль (90) бугра. Если бугор образован движением льдистого материала, то его профиль даже при движении с неизменным ускорением будет такой же, как и профиль скорости. При ламинарном течении вязкой жидкости в трубе профилем скорости является парабола:

$$
v=v_{\max } \cdot\left[1-\left(\frac{r}{R_{h}}\right)^{2}\right] .
$$

Тогда профиль бугра будет иметь вид:

$$
H_{q}=H_{m x} \cdot\left[1-\left(\frac{r}{R_{h}}\right)^{2}\right] .
$$

Профиль скорости, аналогичный профилю бугра (90), получен для вертикальной скорости в сходящемся к центру течении жидкости, например, в пористой трубе с одним заглушенным торцом (см. стр. 59 [31]). Профили (90) и (94) для лучшего сравнения на Рис. 23 нанесены на профиль тени. При этом в выражениях (90) и (94) вместо $H_{m x}$ использована максимальная длина тени $l_{O s h m x}=70.4$ м. Как видно из графиков, профиль (90) для сходящегося течения значительно лучше согласуется с профилем тени, нежели профиль ламинарного течения (94). Имеющееся в правой

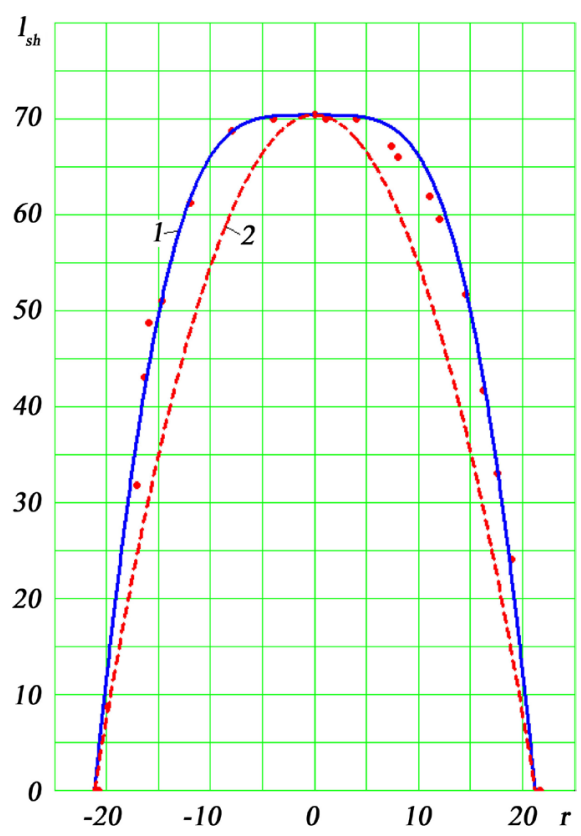

Рис. 23. Сравнение профиля тени с апроксимационными зависимостями: 1-для сходящегося течения (90); 2-для ламинарного течения (94); точки-по результатам измерения тени; $1_{s h}$ и $r$-длина тени бугра и его радиус в м. 
части отличие наблюдаемого профиля тени от зависимости (90) вызвано влиянием снега на изображение правой стороны бугра (Рис. 22(а)).

Природа образования воронки на Ямале неизвестна. В настоящее время существуют разные предположения о причинах ее возникновения. Полученный по тени профиль бугра (90) может быть объяснен стоком пласта льдистого вещества к центру бугра, вследствие чего происходит его вертикальное движение вверх. Насколько возможен такой механизм образования воронки могут показать только дальнейшие ее исследования.

Рассмотренный пример использования феноменов Солнца для аэрокосмических исследований показывает большие потенциальные возможности этого метода.

\section{1. Заключение}

Большие колебания оси вращения Земли объясняют колебания палеоклимата и имеют косвенное подтверждение в некоторых древних календарях и в исторических свидетельствах. Расшифровка древних календарей затруднена сложностью процессов, от которых зависят солнечные явления, фиксируемые календарями. Разработана теория феноменов Солнца, которая алгоритмизирована и реализована в виде программы в среде MathCad. В отличие от традиционной теории, основанной на уравнении Кеплера, в новой теории долгота $\lambda$ годового движения Солнца в зависимости от номера дня года определяется по результатам точного решения задачи двух тел.

Рассчитаны длительности сезонов, долготы светового дня, временные характеристики полярных дней и ночей, азимуты восходов и заходов Солнца, длины теней гномона и их азимуты, а также другие феномены Солнца для современной эпохи и для четырех экстремальных эпох на интервале 50 тысяч лет назад. По современной эпохе результаты подтверждены результатами традиционной теории феноменов Солнца и выполненными наблюдениями.

Подведен итог по основным феноменам Солнца. Рассмотрены алгоритмы восстановления отдельных параметров орбитального и вращательного движения Земли, а также широты и возраста эпохи по древним календарям. Показаны возможности разработанного алгоритма и программы на SunPhnmen.mcd при аэрокосмических исследованиях рельефа Земли.

\section{References}

[1] Дирингская культура (2016) Википедия. https://ru.wikipedia.org/wiki/

[2] Оппенгеймер, С. (2004) Изгнание из Эдема. М.: Из-во Эксмо. - 640 с.

[3] Смульский, И.И. (2014) Основные положения и новые результаты астрономической теории изменения климата. Институт криосферы Земли СО РАН. Тюмень,. - 30 с.: ил: 16. - Библиогр.: 44 назв. - Рус. Деп. в ВИНИТИ РАН 30.09.2014, No. 258-B2014. http://www.ikz.ru/ smulski/Papers/OsPoATLP3.pdf 
[4] Smulsky, J.J. (2016) New Results on the Earth Insolation and Their Correlation with the Late Pleistocene Paleoclimate of West Siberia. Russian Geology and Geophysics, 57, 1099-1110. https://doi.org/10.1016/j.rgg.2016.06.009

[5] Смульский, И.И. (2016) Эволюция оси Земли и палеоклимата за 200 тысяч лет. Saarbrucken, Germany: "LAP Lambert Academic Publishing”, 228 c. http://www.ikz.ru/ smulski/Papers/InfEvEAPC02MEn.pdf

[6] Svendsen, J.I., Astakhov, V.I., Bolshiyanov, D.Y., Demidov, I., Dowdeswell, J.A., Gataflin, V., Hjort, C., Hubberten, H.W., Larsen, E., Mangerud, J., Melles, M., Moller, P., Saarnisto, M. and Siegert, M.J. (1999) Maximum Extent of the Eurasian Ice Sheets in the Barents and Kara Sea Region during the Weichselian. Boreas, 28, 234-242. https://doi.org/10.1111/j.1502-3885.1999.tb00217.x

[7] Флинт, Р.Ф. (1978) История Земли. М.:"Прогресс", 358 с.

[8] Смульский, И.И. (2015) Новые инсоляционные периоды и последние похолодания в плиоцене/В сб. Арктика, Субарктика: мозаичность, контрастность, вариативность криосферы: Труды международной конференции/Под ред. В.П.Мельникова и Д.С.Дроздова. Тюмень: Изд-во Эпоха, С. 360-363. http://www.ikz.ru/ smulski/Papers/smulsky_J_J2015_03_15c1.pdf

[9] Смульский И.И. and Сеченов К.Е. (2007) Уравнения вращательного движения Земли и их решения при воздействии Солнца и планет / Институт криосферы Земли СО РАН. Тюмень, 35 с. - ил.: 7. Библиогр.: 19 назв. - Рус. - Деп. в ВИНИТИ 02.05.07 г. No. 492-В2007. http://www.ikz.ru/ smulski/Papers/UVrVzSPc.pdf

[10] Smulsky J.J. (2011) The Influence of the Planets, Sun and Moon on the Evolution of the Earth's Axis. International Journal of Astronomy and Astrophysics, 1, 117-134. http://www.scirp.org/journal/ijaa/ https://doi.org/10.4236/ijaa.2011.13017

[11] Smul'skii, I.I. (2013) Analyzing the Lessons of the Development of the Orbital Theory of the Paleoclimate. Herald of the Russian Academy of Sciences, 83, 46-54. https://link.springer.com/article/10.1134\%2FS1019331613010073 https://doi.org/10.1134/S1019331613010073

[12] Laskar, J., Robutel, P., Joutel, F., Gastineau, M., Correia, A.C.M. and Levrard, B. (2004) A Long-Term Numerical Solution for the Earth. Icarus, 170, 343-364. https://doi.org/10.1016/j.icarus.2004.04.005

[13] Smulsky, J.J. (2016) Fundamental Principles and Results of a New Astronomic Theory of Climate Change. Advances in Astrophysics, 1, 1-21.

http://www.isaacpub.org

http://www.isaacpub.org/Journal/AdAp https://doi.org/10.22606/adap.2016.11001

[14] Исрапилов, М.И. (2003) Наскальные рисунки Дагестана и колебания полюсов и наклона оси Земли в голоцене. Махачкала: Издательство “Юпитер”. 432 с.

[15] Тилак, Б.Г. (2002) Арктическая родина в Ведах. М.: ФАИР-ПРЕСС., 528 с.

[16] Chippindale, Ch. (1983) Stonehenge Complete: Thames \& Hudson and Ithaca, London.

[17] Mörner, N.-A. and Lind, B.G. (2012) Stonehenge Has Got a Younger Sister Ales Stones in Sweden Decoded. International Journal of Astronomy and Astrophysics, 2, 23-27 https://doi.org/10.4236/ijaa.2012.21004

[18] Ларичев, В.Е. (1989) Мудрость змеи: Первобытный человек, Луна и Солнце. Новосибирск: “Наука”, Сибирское Отделение, 272 с. 
[19] Смульский, И.И. and Кротов, О.И. (2013) Новый алгоритм расчета инсоляции Земли/Институт криосферы Земли СО РАН. - Тюмень, 38 с. - Деп. в ВИНИТИ 08.04.2013 No. 103-B2013.

http://www.ikz.ru/ smulski/Papers/NwAlClI2c.pdf

[20] Smulsky, J.J. and Krotov, O.I. (2014) New Computing Algorithm of the Earths' Insolation. Applied Physics Research, 6, 56-82. https://doi.org/10.5539/apr.v6n4p56

[21] Смульский, И.И. (1999) Теория взаимодействия. - Новосибирск: Из-во Новосиб. ун-та, НИЦ ОИГГМ СО РАН, 294 c. http://www.ikz.ru/ smulski/TVfulA5_2.pdf

[22] Смульский, И.И. (2007) Математическая модель Солнечной системы/В сб. Теоретические и прикладные задачи нелинейного анализа. Российская Академия Наук: ВЦ им. А.А. Дородницына. М.: ВЦ РАН А.А. Дородницына. С. 119-138. http://www.ikz.ru/ smulski/Papers/MatMdSS5.pdf

[23] Справочное руководство по небесной механике и астродинамике (1976) /Под ред. Дубошина Г.Н. Изд. 2-е, доп. и перераб. М., Наука, 862 с.

[24] Труды ИПА РАН. Вып. 10. Эфемеридная астрономия (2004) Санкт-Петербург: ИПА РАН, $488 \mathrm{c}$.

[25] Птолемей, К. (1998) Альмагест. Математическое сочинение в тринадцати книгах. М.: Наука. Физматлит, 672 с.

[26] Ньютон, Р.Р. (1985) Преступление Клавдия Птолемея: пер. с англ. М.: Наука, $384 \mathrm{c}$.

[27] Smulsky, J.J. (2014) Exact Equations for the Light Doppler Effect. Journal of Modern Physics, 5, 1602-1607. https://doi.org/10.4236/jmp.2014.516161

[28] Смульский, И.И. (2016) Феномены Солнца в исторической перспективе/Институт криосферы Земли СО РАН. - Тюмень, 66 с. - Илл.: 23.Библиогр.: 24 назв. - Рус. Деп. в ВИНИТИ РАН 11.01.2016, No. 9-В2016. http://www.ikz.ru/ smulski/Papers/SunPhnmen.pdf

[29] Богоявленский, В.И. and Гарагаш, И.А. (2015) Обоснование процесса образования кратеров газового выброса в Арктике математическим моделированием. Арктика: экология и экономика, No. 3 (19), с. 12-17.

[30] Кизяков, А.И., Сонюшкин, А.В., Лейбман, М.О., Зимин, М.В. and Хомутов, А.В. (2013) Геоморфологические условия образования воронки газового выброса и динамика этой формы на центральном Ямале. Криосфера Земли. T. XVII. No. 4. С. $36-47$.

[31] Смульский, И.И. (1992) Аэродинамика и процессы в вихревых камерах. Новосибирск: ВО "Наука", 301 с. http://www.ikz.ru/ smulski/Aerpro/aerpro.djvu 


\section{“Приложение Программа для расчета феноменов Солнца на основе результатов задачи двух тел"}

SunPhnmen.med. The program for the Sun's phenomena computation on the basis of the two-body problem results.

It is completed 30.12.2015. It is modified 30.12.2015

\section{The constants}

$$
\begin{aligned}
& \mathrm{rd}:=57.29577951308232 \quad \text { ed }:=24 \cdot 3600 \quad \tau:=1440 \quad \mathrm{kJpm} 2:=41.868 \quad \mathrm{k}_{\mathrm{sj}}:=\frac{365.25636042}{365.25} \\
& \mathrm{pi}:=3.141592653589793 \quad \text { p2 }:=2 \cdot \mathrm{pi} \quad \text { pi0 } 5:=0.5 \cdot \mathrm{pi} \quad \text { pi15 }:=1.5 \cdot \mathrm{pi} \\
& \text { Psd }:=365.25636042 \quad \text { Psds }:=\text { Psd } \cdot \text { ed } \quad \Delta \mathrm{dsd}:=\mathrm{Psd}-365 \quad \mathrm{Ptr}:=3.6524219879 \cdot 10^{2} \\
& \mathrm{G}:=6.67259 \cdot 10^{-11} \mathrm{mErt}:=5.9742 \cdot 10^{24} \quad \text { ae }:=1.49597871 \cdot 10^{11} \quad \mathrm{MS}:=332951.3 \cdot \mathrm{mErt} \quad \mathrm{M} 1=-\mathrm{G} \cdot(\mathrm{MS}+\mathrm{mErt}) \\
& \mathrm{Am}:=1.097960770309581 \cdot 10^{13} \quad \mathrm{k}_{\mathrm{V}}:=2.874251102012487 \cdot 10^{-4} \quad \mathrm{Mss}:=1.991787350282 \cdot 10^{30} \\
& \mathrm{am}:=1.362548000293008 \cdot 10^{-2}
\end{aligned}
$$

The parameters of the Earth's orbit and the plane of the equator in epoch 2000 for the Sun's phenomena computation without taking into account the change of the Earth's orbital and rotational motions.

$$
\begin{aligned}
& \text { ec } 0=1.67086342 \cdot 10^{-2} \quad \varepsilon 0:=0.409092629689404 \quad \text { fpy } 0:=1.796595647267463 \quad \text { Tp } 0:=0.500143001 \\
& \mathrm{JD} 49:=2433280.5 \quad \mathrm{JDp} 0:=\mathrm{JD} 49+\mathrm{Tp} 0 \cdot 100 \cdot \text { Psd } \quad \mathrm{JDp} 0=2.451548541223479 \cdot 10^{6}
\end{aligned}
$$

\section{Reading of the parameters of the Earth's orbital} and rotational motions from the files.

The file of Laskar J. et al [12]. The time T2 is in thousand years.

$$
\begin{aligned}
& \text { R1 := RrrrEADPRN("INSO_LA2004.txt") N41:= } 200 \mathrm{di}:=10 \\
& \mathrm{~T} 2_{\mathrm{i} 4}:=10 \cdot \mathrm{R} 1_{\mathrm{i} 4 \cdot \mathrm{di}, 0} \quad \mathrm{ec}_{\mathrm{i} 4}:=\mathrm{R} 1_{\mathrm{i} 4 \cdot \mathrm{di}, 1} \quad \varepsilon_{\mathrm{i} 4}:=\mathrm{R} 1_{\mathrm{i} 4 \cdot \mathrm{di}, 2} \quad \mathrm{fpy} y_{\mathrm{i} 4}:=\mathrm{R} 1_{\mathrm{i} 4 \cdot \mathrm{di}, 3} \\
& \text { The files of Smulsky J.J. The time T2 is in sidereal centuries since } 30.12 .1949 \text {. } \\
& 2500 \quad 5000 \\
& \text { "OrAl1c_8.prn" "OrAl-200ky.prn" } \\
& \mathrm{R} 1:=\text { READPRN("OrAl-200ky.prn") } \quad \mathrm{N} 410:=5000 \quad \mathrm{di}:=1 \quad \mathrm{~N} 40:=\frac{\mathrm{N} 410}{\mathrm{di}} \quad \mathrm{i} 40:=0 . . \mathrm{N} 40 \quad \mathrm{i} 50:=1625 \\
& \mathrm{~T} 20_{\mathrm{i} 40}:=\mathrm{R} 1_{\mathrm{i} 40 \cdot \mathrm{di}, 0} \quad \text { ec00 } 0_{\mathrm{i} 40}:=\mathrm{R} 1_{\mathrm{i} 40 \cdot \mathrm{di}, 1} \quad \quad f p y 00_{\mathrm{i} 40}:=\mathrm{R} 1_{\mathrm{i} 40 \cdot \mathrm{di}, 2} \quad \mathrm{di} \quad \mathrm{T} 20_{\mathrm{N} 40}=-2 \cdot 10^{3} \\
& \varepsilon 00_{\mathrm{i} 40}:=\mathrm{R} 1_{\mathrm{i} 40 \cdot \mathrm{di}, 3} \quad \mathrm{JD} 0_{\mathrm{i} 40}:=\mathrm{R} 1_{\mathrm{i} 40 \cdot \mathrm{di}, 4} \quad \mathrm{~T} 0_{\mathrm{jy}} \mathrm{i}_{\mathrm{i} 40}:=\mathrm{k}_{\mathrm{sj}} \cdot \mathrm{T} 20_{\mathrm{i} 40} \cdot 100 \quad \mathrm{~T} 20_{\mathrm{i} 50}=-6.5 \cdot 10^{2} \\
& \mathrm{i} 4 \mathrm{I}_{0}:=0 \quad \mathrm{i} 4 \mathrm{I}_{1}:=104 \quad \mathrm{i}_{4} \mathrm{I}_{2}:=397 \quad \mathrm{i}_{4} \mathrm{I}_{3}:=782 \quad \mathrm{i} 4 \mathrm{I}_{4}:=1161 \\
& \mathrm{i} 4 \mathrm{o}_{0}:=0 \quad \mathrm{i} 4 \mathrm{o}_{1}:=70 \quad \mathrm{i} 4 \mathrm{o}_{2}:=383 \quad \mathrm{i} 4 \mathrm{o}_{3}:=775 \quad \mathrm{i} 4 \mathrm{o}_{4}:=1161 \\
& \mathrm{~N} 4:=4 \quad \mathrm{i} 4:=0 \text {.. N4 i5 }:=0 \\
& \mathrm{~T} 2_{\mathrm{i} 4}:=\mathrm{T}_{2} \mathrm{i}_{\mathrm{i} 4 \mathrm{o}_{14}} \quad \mathrm{ec}_{\mathrm{i} 4}:=\mathrm{ec} 00_{\mathrm{i} 4 \mathrm{o}_{\mathrm{i} 4}} \quad \mathrm{fp} \gamma_{\mathrm{i} 4}:=\mathrm{fp} / 00_{\mathrm{i} 4 \mathrm{o}_{\mathrm{i} 4}} \quad \varepsilon_{\mathrm{i} 4}:=\varepsilon 00_{\mathrm{i} 4 \mathrm{o}_{\mathrm{i} 4}} \quad \mathrm{JD}_{\mathrm{i} 4}:=\mathrm{JD} 0_{\mathrm{i}_{4} \mathrm{o}_{\mathrm{i} 4}} \quad \mathrm{~T}_{\mathrm{jy}}:=\mathrm{T}_{\mathrm{i} 4} 0_{\mathrm{jyi} 4 \mathrm{o}_{\mathrm{i} 4}} \quad \mathrm{~T} 2_{\mathrm{i} 5}=0
\end{aligned}
$$


3. The trajectory parameter, the perihelion radius, the velocity at the perihelion and the movement time to the aphelion

$\mathrm{all}_{\mathrm{i} 4}:=\frac{-1}{1+\mathrm{ec}_{\mathrm{i} 4}} \quad \mathrm{Rp}_{\mathrm{i} 4}:=\mathrm{am} \cdot \frac{\left(2 \cdot \mathrm{all} 1_{\mathrm{i} 4}+1\right)}{\mathrm{all}_{\mathrm{i} 4}} \quad \mathrm{vp}_{\mathrm{i} 4}:=\frac{-\mathrm{p} 2 \cdot \mathrm{all} 1_{\mathrm{i} 4} \cdot \mathrm{Rp}_{\mathrm{i} 4} \cdot \mathrm{Am}}{\operatorname{Psds} \cdot\left(-2 \cdot \mathrm{all}_{\mathrm{i} 4}-1\right)^{1.5}} \quad \mathrm{ta}_{\mathrm{i} 4}:=-\left(\frac{\mathrm{Rp}_{\mathrm{i} 4} \cdot \mathrm{Am}}{\mathrm{vp}_{\mathrm{i} 4}}\right) \cdot \frac{\pi \cdot \mathrm{all} \mathrm{i}_{\mathrm{i}}}{\left(-2 \cdot \mathrm{all}_{\mathrm{i} 4}-1\right)^{1.5}}$

4. The polar angle and time of the Sun's motion in the plane of its orbit according to the two-body problem

$\mathrm{J}$ is the number of parts in a year or points of the the Sun's longitude.

$\lambda 0$ is a series of the Sun's longitudes with members, regular spaced by the value $\mathrm{d} \lambda 0$.

$\lambda$ is a series of the longitudes with members, regular spaced in time.

$\mathrm{fi}$ is the polar angle (anomaly), and ra0 is the radius of the Sun's motion.

$v$ is the longitude of the Sun's perigee from the point of vernal equinox.

$\mathrm{tp} 0$ is the time of the Sun's movement from the perigee to the point with the polar angle fi.

tpy is the time of the Sun's movement from the perigee to the point of vernal equinox.

$$
\begin{aligned}
& \mathrm{J}:=365 \quad \mathrm{~J} 0:=365 \quad \mathrm{~d} \lambda 0:=\frac{2 \cdot \pi}{\mathrm{J}} \quad \lambda 0_{0}:=0 \quad \mathrm{j} 0:=0 \ldots \mathrm{J} \quad \mathrm{j} 1:=1 \ldots \mathrm{J} \quad \mathrm{j} 3:=1 \ldots \mathrm{J}+1 \quad \mathrm{j} 03:=0 \ldots \mathrm{J}+1 \\
& \lambda 0_{\mathrm{j} 1}:=\lambda 0_{\mathrm{j} 1-\mathrm{l}}+\mathrm{d} \lambda 0 \quad \lambda 0_{\mathrm{J}+\mathrm{l}}:=\lambda 0_{\mathrm{J}}+\mathrm{d} \lambda 0 \quad \lambda 0_{0}=0 \quad \lambda 0_{\mathrm{J}}=6.283185307179618 \\
& v 0_{\mathrm{i} 4}:=\mathrm{fp} \gamma_{\mathrm{i} 4}+\pi \quad v 0 \mathrm{~T}_{\mathrm{i} 4}:=\text { floor }\left(\frac{v 0_{\mathrm{i} 4}}{\mathrm{p} 2}\right) \quad v_{\mathrm{i} 4}:=v 0_{\mathrm{i} 4}-v 0 \mathrm{~T}_{\mathrm{i} 4} \cdot \mathrm{p} 2 \quad \quad \mathrm{fi} 0_{\mathrm{i} 4, \mathrm{j} 03}:=\lambda 0_{\mathrm{j} 03}-v_{\mathrm{i} 4} \\
& \mathrm{fi}_{\mathrm{i} 4, \mathrm{j} 03}:=\mathrm{if}\left(\mathrm{fi}_{\mathrm{i} 4, \mathrm{j} 03}<0, \mathrm{fi} 0_{\mathrm{i} 4, \mathrm{j} 03}+\mathrm{p} 2, \mathrm{fi} 0_{\mathrm{i} 4, \mathrm{j} 03}\right) \quad \mathrm{fi}_{\mathrm{i} 4, \mathrm{j} 03}:=\mathrm{if}\left(\mathrm{fi}_{\mathrm{i} 4, \mathrm{j} 03}>\mathrm{p} 2, \mathrm{fi}_{\mathrm{i} 4, \mathrm{j} 03}-\mathrm{p} 2, \mathrm{fi} 1_{\mathrm{i} 4, \mathrm{j} 03}\right) \\
& \mathrm{ra}_{\mathrm{i} 4, \mathrm{j} 03}:=\frac{\mathrm{Rp}_{\mathrm{i} 4} \cdot \mathrm{Am}}{\left[\left(\mathrm{all}_{\mathrm{i} 4}+\mathbf{1}\right) \cdot \cos \left(\mathrm{fi}_{\mathrm{i} 4, \mathrm{j} 03}\right)-\mathrm{all}_{\mathrm{i} 4}\right]}
\end{aligned}
$$

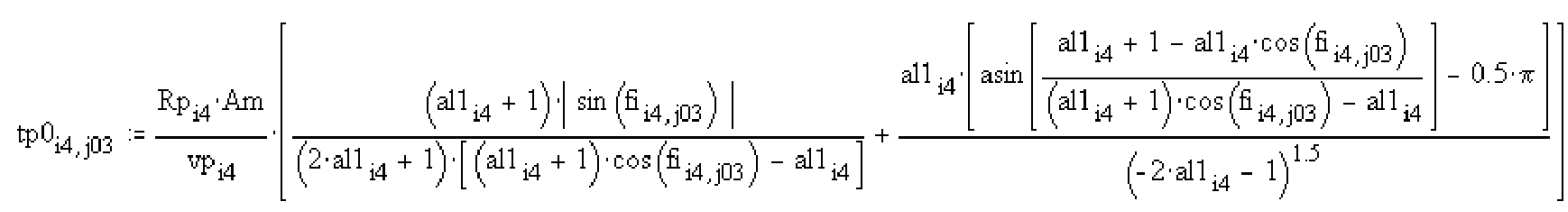

$$
\begin{aligned}
& \operatorname{tp}_{\mathrm{i} 4, \mathrm{j} 03}:=\mathrm{if}\left(\mathrm{fi}_{\mathrm{i} 4, \mathrm{j} 03}>\pi, 2 \cdot \mathrm{ta}_{\mathrm{i} 4}-\mathrm{tp} 0_{\mathrm{i} 4, \mathrm{j} 03}, \mathrm{tp} 0_{\mathrm{i} 4, \mathrm{j} 03}\right) \quad \quad \mathrm{tp}_{\mathrm{i} 4, \mathrm{j} 03}:=\text { if }\left(\lambda 0_{\mathrm{j} 03}>v_{\mathrm{i} 4}, 2 \cdot \mathrm{ta}_{\mathrm{i} 4}+\mathrm{tp}_{\mathrm{i} 4, \mathrm{j} 03}, \mathrm{tp}_{\mathrm{i} 4, \mathrm{j} 03}\right)
\end{aligned}
$$

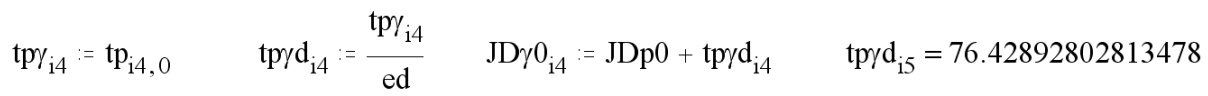

5. The computation of longitudes with members, regularly spaced in time.

The first refinement of a series of longitudes

$$
\begin{aligned}
& \operatorname{Td}_{\mathrm{j} 3}:=\mathrm{j} 3 \quad \lambda \mathrm{i}_{\mathrm{j} 03}:=0 \quad \lambda \mathrm{i}_{\mathrm{i} 4, \mathrm{j} 0+1}:=\lambda \mathrm{i}_{\mathrm{i} 4, \mathrm{j} 0}+\frac{\lambda 0_{\mathrm{j} 0+1}-\lambda 0_{\mathrm{j} 0}}{\operatorname{tp} \mathbf{1}_{\mathrm{i} 4, \mathrm{j} 0+1}-\mathrm{tp} \mathbf{1}_{\mathrm{i} 4, \mathrm{j} 0}} \cdot 1 \cdot \mathrm{ed} \\
& \mathrm{fi2} 2_{14, \mathrm{j} 03}:=\lambda \mathrm{i}_{\mathrm{i} 4, \mathrm{j} 03}-v_{\mathrm{i} 4} \quad \mathrm{fi} 3_{\mathrm{i} 4, \mathrm{j} 03}:=\text { if }\left(\mathrm{fi} 2_{\mathrm{i} 4, \mathrm{j} 03}<0, \mathrm{fi} 2_{\mathrm{i} 4, \mathrm{j} 03}+\mathrm{p} 2, \mathrm{fi} 2_{\mathrm{i} 4, \mathrm{j} 03}\right) \quad \mathrm{fi} 4_{\mathrm{i} 4, \mathrm{j} 03}:=\text { if }\left(\mathrm{fi} 3_{\mathrm{i} 4, \mathrm{j} 03}>\mathrm{p} 2, \mathrm{fi} 3_{\mathrm{i} 4, \mathrm{j} 03}-\mathrm{p} 2, \mathrm{fi} 3_{\mathrm{i} 4, \mathrm{j} 03}\right)
\end{aligned}
$$

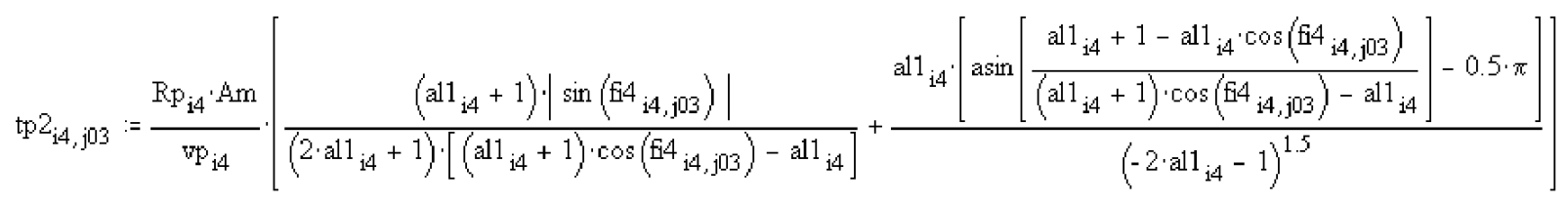




$$
\operatorname{tp}_{\mathrm{i} 4, \mathrm{j} 03}:=\mathrm{if}\left(\mathrm{fi} 4_{\mathrm{i} 4, \mathrm{j} 03}>\pi, 2 \cdot \mathrm{ta}_{\mathrm{i} 4}-\mathrm{tp} 2_{\mathrm{i} 4, \mathrm{j} 03}, \mathrm{tp} 2_{\mathrm{i} 4, \mathrm{j} 03}\right) \quad \operatorname{tp} 4_{\mathrm{i} 4, \mathrm{j} 03}:=\text { if }\left(\lambda \mathrm{i}_{\mathrm{i} 4, \mathrm{j} 03}>v_{\mathrm{i} 4}, 2 \cdot \mathrm{ta} \mathrm{i}_{14}+\mathrm{tp} 3_{\mathrm{i} 4, \mathrm{j} 03}, \mathrm{tp} 3_{\mathrm{i} 4, \mathrm{j} 03}\right)
$$

The second refinement of a series of longitudes

$$
\begin{aligned}
& \lambda \mathbf{i} 2_{\mathrm{i} 4, \mathrm{j} 03}:=0 \quad \lambda \mathrm{i} 2_{\mathrm{i} 4, \mathrm{j} 0+1}:=\lambda \mathrm{i} 2_{\mathrm{i} 4, \mathrm{j} 0}+\frac{\lambda \mathrm{i}_{\mathrm{i} 4, \mathrm{j} 0+1}-\lambda \mathrm{i}_{\mathrm{i} 4, \mathrm{j} 0}}{\operatorname{tp} 4_{\mathrm{i} 4, \mathrm{j} 0+1}-\mathrm{tp} 4_{\mathrm{i} 4, \mathrm{j} 0}} \cdot 1 \cdot \mathrm{ed} \\
& \mathrm{fi}_{\mathrm{i} 4, \mathrm{j} 03}:=\lambda \mathrm{i} 2_{\mathrm{i} 4, \mathrm{j} 03}-v_{\mathrm{i} 4} \quad \mathrm{fi} 6_{\mathrm{i} 4, \mathrm{j} 03}:=\mathrm{if}\left(\mathrm{fi}_{\mathrm{i} 4, \mathrm{j} 03}<0, \mathrm{fi} 5_{\mathrm{i} 4, \mathrm{j} 03}+\mathrm{p} 2, \mathrm{fi} 5_{\mathrm{i} 4, \mathrm{j} 03}\right) \quad \text { fi1 }{ }_{\mathrm{i} 4, \mathrm{j} 03}:=\mathrm{if}\left(\mathrm{fi}_{\mathrm{i} 4, \mathrm{j} 03}>\mathrm{p} 2, \mathrm{fi} 6_{\mathrm{i} 4, \mathrm{j} 03}-\mathrm{p} 2, \mathrm{fi} 6_{\mathrm{i} 4, \mathrm{j} 03}\right)
\end{aligned}
$$

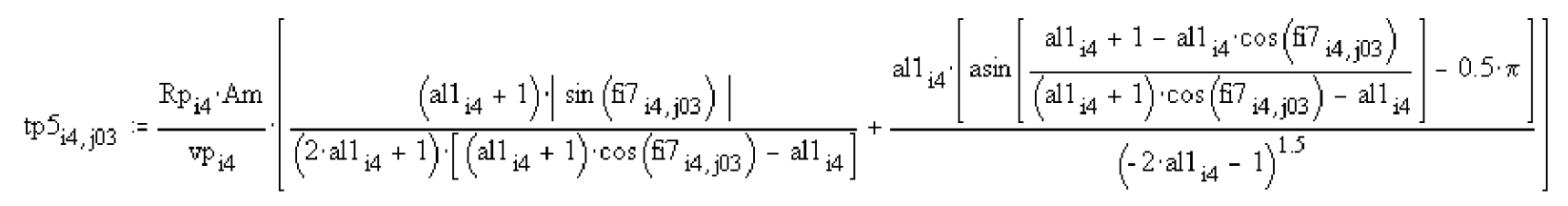

$$
\begin{aligned}
& \operatorname{tp}_{\mathrm{i} 4, \mathrm{j} 03}:=\text { if }\left(\mathrm{fi}_{\mathrm{i} 4, \mathrm{j} 03}>\pi, 2 \cdot \mathrm{ta} \mathrm{i}_{4}-\mathrm{tp}_{\mathrm{i} 4, \mathrm{j} 03}, \mathrm{tp} 5_{\mathrm{i} 4, \mathrm{j} 03}\right) \quad \operatorname{tp} 7_{\mathrm{i} 4, \mathrm{j} 03}:=\mathrm{if}\left(\lambda \mathrm{i} 2_{\mathrm{i} 4, \mathrm{j} 03}>v_{\mathrm{i} 4}, 2 \cdot \mathrm{ta}_{\mathrm{i} 4}+\mathrm{tp} 6_{\mathrm{i} 4, \mathrm{j} 03}, \mathrm{tp} 6_{\mathrm{i} 4, \mathrm{j} 03}\right)
\end{aligned}
$$

The third refinement of a series of longitudes

$$
\begin{gathered}
\lambda_{\mathrm{i} 4, \mathrm{j} 03}:=0 \quad \lambda_{\mathrm{i} 4, \mathrm{j} 0+1}:=\lambda_{\mathrm{i} 4, \mathrm{j} 0}+\frac{\lambda \mathrm{i} 2_{\mathrm{i} 4, \mathrm{j} 0+1}-\lambda \mathrm{i} 2_{\mathrm{i} 4, \mathrm{j} 0}}{\mathrm{tp} 7_{\mathrm{i} 4, \mathrm{j} 0+1}-\mathrm{tp} 7_{\mathrm{i} 4, \mathrm{j} 0}} \cdot \mathrm{ed} \\
\mathrm{fi} 8_{\mathrm{i} 4, \mathrm{j} 3}:=\lambda_{\mathrm{i} 4, \mathrm{j} 3}-v_{\mathrm{i} 4} \quad \mathrm{fi} 9_{\mathrm{i} 4, \mathrm{j} 3}:=\mathrm{if}\left(\mathrm{fi} 8_{\mathrm{i} 4, \mathrm{j} 3}<0, \mathrm{fi} 8_{\mathrm{i} 4, \mathrm{j} 3}+\mathrm{p} 2, \mathrm{fi} 8_{\mathrm{i} 4, \mathrm{j} 3}\right) \quad \text { fi } 10_{\mathrm{i} 4, \mathrm{j} 3}:=\text { if }\left(\mathrm{fi} 9_{\mathrm{i} 4, \mathrm{j} 3}>\mathrm{p} 2, \mathrm{fi} 9_{\mathrm{i} 4, \mathrm{j} 3}-\mathrm{p} 2, \mathrm{fi} 9_{\mathrm{i} 4, \mathrm{j} 3}\right)
\end{gathered}
$$

The check of intervals at refinement series of longitudes

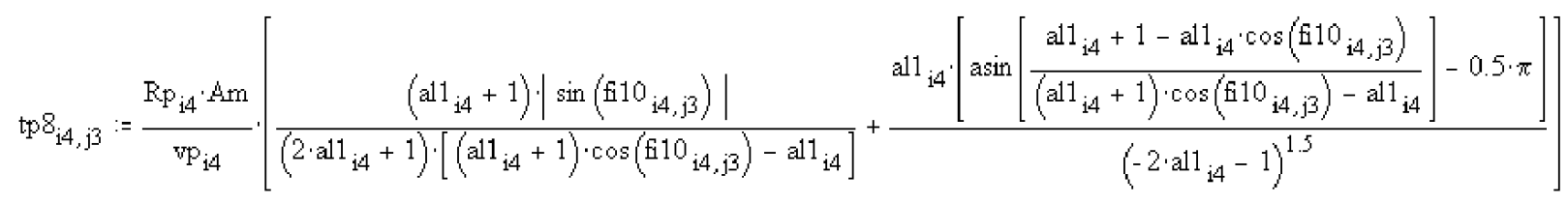

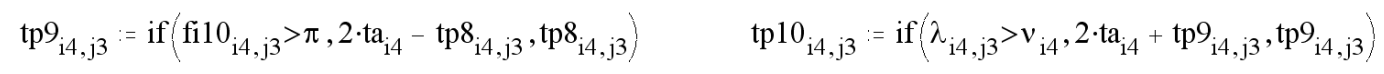

$$
\begin{aligned}
& \mathrm{dtp} 3_{\mathrm{i} 4, \mathrm{j} 1}:=\frac{\left(\operatorname{tp} 10_{\mathrm{i} 4, \mathrm{j} 1+1}-\operatorname{tp} 10_{\mathrm{i} 4, \mathrm{j} 1}\right)-\mathrm{ed}}{\mathrm{ed}} \operatorname{ddtpS} 3_{\mathrm{i} 4,0}:=0 \quad \operatorname{ddtpS} 3_{\mathrm{i} 4, \mathrm{j} 1}:=\operatorname{ddtpS} 3_{\mathrm{i} 4, \mathrm{j} 1-1}+\left|\mathrm{dtp} 3_{\mathrm{i} 4, \mathrm{j} 1}\right| \\
& \frac{\mathrm{ddtpS}_{\mathrm{i} 5, \mathrm{~J}}}{\mathrm{~J}}=7.155883412539792 \cdot 10^{-7} \quad \mathrm{~T} 2_{\mathrm{i} 5}=0
\end{aligned}
$$

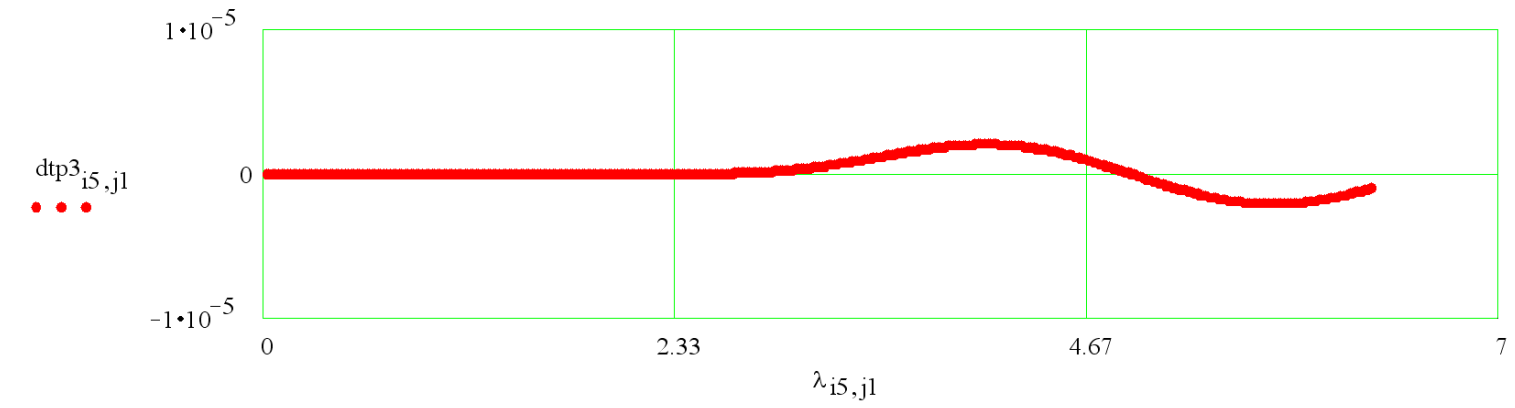

The errors of the year continuance definition.

The days definition accuracy check in a sidereal year: $\Delta \mathrm{dsd}$ is the day residual until the end of a sidereal year.

$\Delta \mathrm{dsdc}:=\frac{\left(\mathrm{p} 2-\lambda_{\mathrm{i} 5, \mathrm{~J}}\right) \cdot 1}{\lambda_{\mathrm{i} 5, \mathrm{~J}}-\lambda_{\mathrm{i} 5, \mathrm{~J}-1}} \quad \Delta \Delta \mathrm{dg}:=\Delta \mathrm{dsdc}-\Delta \mathrm{dsd} \quad \Delta \Delta \mathrm{dg}=-1.273550658583145 \cdot 10^{-4} \quad \Delta \Delta \mathrm{dg} \cdot \mathrm{ed}=-11.00347769015837$ 
$\delta \lambda_{365}:=\frac{\Delta \mathrm{dsdc}-\Delta \mathrm{dsd}}{\Delta \mathrm{dsd}} \quad \delta \lambda_{365}=-4.967813122567979 \cdot 10^{-4}$

The radius ra of Sun's motion along the orbit depending on a series of longitudes with members, regularly spaced in time.

$$
\mathrm{ra}_{\mathrm{i} 4, \mathrm{j} 1}:=\frac{\mathrm{Rp}_{\mathrm{i} 4} \cdot \mathrm{Am}}{\left[\left(\mathrm{all}_{\mathrm{i} 4}+1\right) \cdot \cos \left(\lambda_{\mathrm{i} 4, \mathrm{j} 1}-v_{\mathrm{i} 4}\right)-\mathrm{all} 1_{\mathrm{i} 4}\right]} \quad \rho_{\mathrm{i} 4, \mathrm{j} 1}:=\frac{\mathrm{ra}_{\mathrm{i} 4, \mathrm{j} 1}}{\mathrm{ae}}
$$

6. Setting the location of the observer's latitude.

\begin{tabular}{|c|c|c|c|c|}
\hline $\mathrm{i} 1:=1 .$. If $+1 \quad \phi_{0}:=-\frac{2}{\mathrm{rd}}$ & $\phi_{\mathrm{il}}:=\phi_{\mathrm{il}-1}+\frac{2}{\mathrm{rd}}$ & $\phi g_{i 1}:=\phi_{i 1} \cdot r d$ & i1 $1:=36$ & 29 \\
\hline Moscow & Tyumen & Bovanenkovo & & \\
\hline 55.75222 & 57.152199 & 70.3622 & & $\phi \mathrm{g}_{\mathrm{i} 11}:=70.3622$ \\
\hline 0.973059804296233 & 0.997494047305011 & 1.228052058946754 & & $\phi_{\mathrm{i} 11}:=1.228052058946754$ \\
\hline
\end{tabular}

7. Sun's declination and the hour angle of sunrises and sunsets.

If is the number of points on geographical latitudes.

i11 is the index of a concrete value of the latitude.

$\phi$ is the geographical latitude of place.

$\delta$ is the Sun's declination.

$\omega 0$ is the hour angle of sunrises and sunsets.

$$
\begin{aligned}
& \sin \delta_{\mathrm{i} 4, \mathrm{j} 1}:=\sin \left(\varepsilon_{\mathrm{i} 4}\right) \cdot \sin \left(\lambda_{\mathrm{i} 4, \mathrm{j} 1}\right) \quad \delta_{\mathrm{i} 4, \mathrm{j} 1}:=\operatorname{asin}\left(\sin \delta_{\mathrm{i} 4, \mathrm{j} 1}\right) \quad \cos \delta_{\mathrm{i} 4, \mathrm{j} 1}=\cos \left(\delta_{\mathrm{i} 4, \mathrm{j} 1}\right) \quad \tan \delta_{\mathrm{i} 4, \mathrm{j} 1}:=\tan \left(\delta_{\mathrm{i} 4, \mathrm{j} 1}\right) \quad \phi \mathrm{g}_{\mathrm{i} 11}=70.3622 \\
& \mathrm{Fn} 00_{\mathrm{i} 4, \mathrm{j} 1}:=-\tan \delta_{\mathrm{i} 4, \mathrm{j} 1} \cdot \tan \left(\phi_{\mathrm{i} 11}\right) \quad \mathrm{Fn} 0_{\mathrm{i} 4, \mathrm{j} 1}:=\text { if }\left(-1<\mathrm{Fn} 00_{\mathrm{i} 4, \mathrm{j} 1}, \mathrm{Fn} 00_{\mathrm{i} 4, \mathrm{j} 1},-1\right) \quad \mathrm{Fn} 0_{\mathrm{i} 4, \mathrm{j} 1}:=\mathrm{if}\left(1>\mathrm{Fn} 0_{\mathrm{i} 4, \mathrm{j} 1}, \mathrm{Fn} 0_{\mathrm{i} 4, \mathrm{j} 1}, 1\right) \\
& \omega 0_{\mathrm{i} 4, \mathrm{j} 1}:=\operatorname{acos}\left(\mathrm{Fn} 0_{\mathrm{i} 4, \mathrm{j} 1}\right)
\end{aligned}
$$

8. Longitude $\lambda$ of the beginning seasons, number of days

Td before they start and length of the seasons.

\section{Beginning summer}

$$
\begin{aligned}
& \operatorname{Ind} 01_{i 4, j 1}:=\operatorname{if}\left(\lambda_{i 4, j 1}>\text { pi05, } 0, j 1\right) \\
& \text { in } 01:=\text { for } i 4 \in 0.1 \mathrm{~N} 4 \\
& \mid \begin{array}{l}
\max \leftarrow 0 \\
\text { for } \mathrm{j} 1 \in 1 \ldots \mathrm{J} \\
{\operatorname{in} 01 \mathrm{a}_{\mathrm{i} 4}-\operatorname{Ind} 01_{\mathrm{i} 4, \mathrm{j} 1} \text { if } \max \leq \operatorname{Ind} 01_{\mathrm{i} 4, \mathrm{j} 1}}_{\max \leftarrow \operatorname{Ind} 01_{\mathrm{i} 4, \mathrm{j} 1} \text { if } \max \leq \operatorname{Ind} 01_{\mathrm{i} 4, \mathrm{j} 1}} \\
\mathrm{in} 01_{\mathrm{a}}
\end{array}
\end{aligned}
$$$$
\mathrm{Td}_{\mathrm{Su}_{\mathrm{i} 4}}:=\mathrm{Td}_{\mathrm{in}_{\mathrm{i} 4}}+\frac{\mathrm{pi05}-\lambda_{\mathrm{i} 4, \mathrm{in}_{\mathrm{i} 4}}}{\lambda_{\mathrm{i}_{4}, \mathrm{in}_{\mathrm{i} 4}+1}-\lambda_{\mathrm{i} 4, \mathrm{in}_{\mathrm{i} 4}}}
$$

\section{Beginning winter}

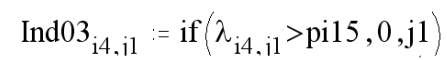

\section{Beginning autum}

$$
\operatorname{Ind} 02_{\mathrm{i} 4, \mathrm{j} 1}:=\mathrm{if}\left(\lambda_{\mathrm{i} 4, \mathrm{j} 1}>\mathrm{pi}, 0, \mathrm{j} 1\right)
$$$$
\text { in } 02:=\text { for } i 4 \in 0 ., \mathrm{N} 4
$$

$$
\mid \begin{aligned}
& \max \leftarrow 0 \\
& \text { for } j 1 \in 1 . . \mathrm{J} \\
& \left.\right|_{\min 02 \mathrm{a}_{\mathrm{i} 4}-\operatorname{Ind} 02_{\mathrm{i} 4}, \mathrm{j} 1} \text { if } \max \leq \operatorname{Ind} 02_{\mathrm{i} 4, \mathrm{j} 1} \\
& \max \leftarrow \operatorname{Ind} 02_{\mathrm{i} 4, \mathrm{j} 1} \text { if } \max \leq \operatorname{Ind} 02_{\mathrm{i} 4, \mathrm{j} 1}
\end{aligned}
$$

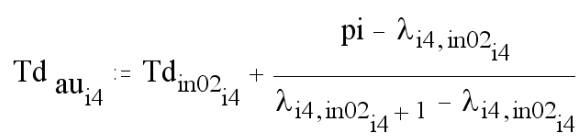




$$
\begin{aligned}
& \text { in } 03:=\text { for } i 4 \in 0 . . \mathrm{N} 4 \\
& \mid \begin{array}{l}
\max \leftarrow 0 \\
\text { for } j 1 \in 1 \ldots \mathrm{J} \\
\left.\right|_{\mathrm{in} 03_{\mathrm{i} 4}-\operatorname{Ind} \mathrm{d} 03_{\mathrm{i} 4, \mathrm{j} 1} \text { if } \max \leq \operatorname{Ind} 003_{\mathrm{i} 4, \mathrm{j} 1}} ^{\max \leftarrow \operatorname{Ind}_{\mathrm{a}} 03_{\mathrm{i} 4, \mathrm{j} 1} \text { if } \max \leq \operatorname{Ind} 03_{\mathrm{i} 4, \mathrm{j} 1}}
\end{array}
\end{aligned}
$$

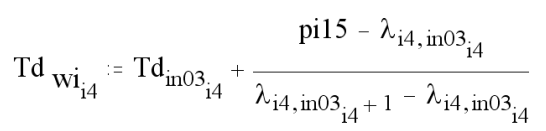$$
\mathrm{Td}_{\mathrm{su}}=\left[\begin{array}{r}
92.77029599636545 \\
94.22585401187585 \\
88.48370457936125 \\
91.19072475675256 \\
92.80560303813662
\end{array}\right]
$$$$
\mathrm{Td}_{\mathrm{au}}=\left[\begin{array}{c}
1.864247021690474 \cdot 10^{2} \\
1.860292802840937 \cdot 10^{2} \\
1.812758745783055 \cdot 10^{2} \\
1.79887367985595 \cdot 10^{2} \\
1.858311036206739 \cdot 10^{2}
\end{array}\right.
$$$$
\mathrm{Td}_{\mathrm{wi}}=\left[\begin{array}{c}
2.762613818306462 \cdot 10^{2} \\
2.744835824248672 \cdot 10^{2} \\
2.754583577418334 \cdot 10^{2} \\
2.712798468644718 \cdot 10^{2} \\
2.756491980312958 \cdot 10^{2}
\end{array}\right]
$$

Length of the seasons

$$
\Delta \mathrm{Td}_{\mathrm{sp}}:=\mathrm{Td}_{\mathrm{su}} \quad \Delta \mathrm{Td}_{\mathrm{su}}:=\mathrm{Td}_{\mathrm{au}}-\mathrm{Td}_{\mathrm{su}} \quad \Delta \mathrm{Td}_{\mathrm{au}}:=\mathrm{Td}_{\mathrm{wi}}-\mathrm{Td}_{\mathrm{au}} \quad \Delta \mathrm{Td}_{\mathrm{wi}}:=\mathrm{Ptr}-\mathrm{Td}_{\text {wi }}
$$

$$
\Delta \mathrm{Td}_{\mathrm{sp}}=\left[\begin{array}{c}
92.77029599636545 \\
94.22585401187585 \\
88.48370457936125 \\
91.19072475675256 \\
92.80560303813662
\end{array}\right] \Delta \mathrm{Td}_{\mathrm{su}}=\left[\begin{array}{c}
93.6544061726819 \\
91.80342627221781 \\
92.79216999894422 \\
88.69664322884242 \\
93.02550058253725
\end{array}\right] \Delta \mathrm{Td}_{\mathrm{au}}=\left[\begin{array}{l}
89.83667966159885 \\
88.45430214077356 \\
94.18248316352796 \\
91.39247887887686 \\
89.81809441062197
\end{array}\right]
$$

$\mathrm{P}_{\mathrm{i} 4,0}:=\mathrm{i} 4+1 \quad \mathrm{P}_{\mathrm{i} 4,1}:=0.1 \cdot \mathrm{T}_{\mathrm{i} 4} \quad \mathrm{P}_{\mathrm{i} 4,2}:=\mathrm{Td}_{\mathrm{su}_{\mathrm{i} 4}} \mathrm{P}_{\mathrm{i} 4,3}:=\mathrm{Td}_{\mathrm{au}_{\mathrm{i} 4}} \quad \mathrm{P}_{\mathrm{i} 4,4}:=\mathrm{Td}_{\mathrm{wi}_{\mathrm{i} 4}}$

$\mathrm{P}_{\mathrm{i} 4,5}:=\Delta \mathrm{Td}_{\mathrm{sp}_{\mathrm{i} 4}} \mathrm{P}_{\mathrm{i} 4,6}:=\Delta \mathrm{Td}_{\mathrm{su}_{14}} \quad \mathrm{P}_{\mathrm{i} 4,7}:=\Delta \mathrm{Td}_{\mathrm{au}_{\mathrm{i} 4}} \mathrm{P}_{\mathrm{i} 4,8}:=\Delta \mathrm{Td}_{\mathrm{wi}_{\mathrm{i} 4}} \mathrm{P}_{\mathrm{i} 4,9}:=\varepsilon_{\mathrm{i} 4}$

$\Delta \mathrm{Td}_{\mathrm{Wi}}=\left[\begin{array}{c}88.98081695935377 \\ 90.75861636513275 \\ 89.78384104816655 \\ 93.96235192552814 \\ 89.59300075870414\end{array}\right]$

WwwwRITEPRN("Seasons.prn" ) := P1

\section{Length of apparent solar day (earthday) and the equation of time.}

$$
\begin{aligned}
& \alpha_{\mathrm{S}_{\mathrm{j} 1}}:=\operatorname{asin}\left[\frac{\cos \left(\varepsilon_{\mathrm{i} 5}\right) \cdot \sin \left(\lambda_{\mathrm{i} 5, \mathrm{j} 1}\right)}{\sqrt{1-\left(\sin \left(\varepsilon_{\mathrm{i} 5}\right) \cdot \sin \left(\lambda_{\mathrm{i} 5, \mathrm{j} 1}\right)\right)^{2}}}\right] \\
& \alpha_{\mathrm{S}_{\mathrm{j} 1}}:=\alpha^{\alpha} \mathrm{S}_{\mathrm{j} 1} \quad \alpha_{\mathrm{S}_{\mathrm{j} 1}}:=\mathrm{if}\left(\lambda_{\mathrm{i} 5, \mathrm{j} 1}>0.5 \cdot \mathrm{pi}, \mathrm{pi}-\alpha_{\mathrm{S}_{\mathrm{j} 1}}, \alpha \mathrm{S}_{\mathrm{j} 1}\right)
\end{aligned}
$$

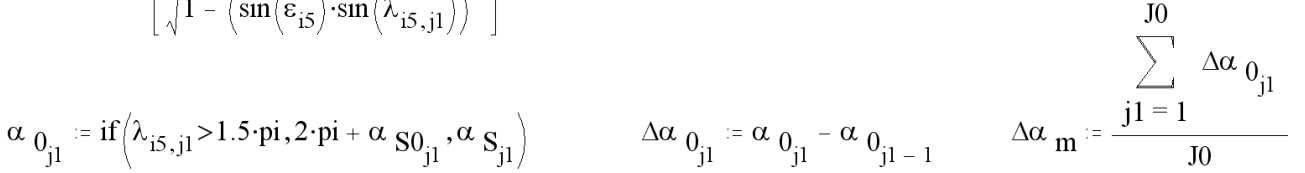

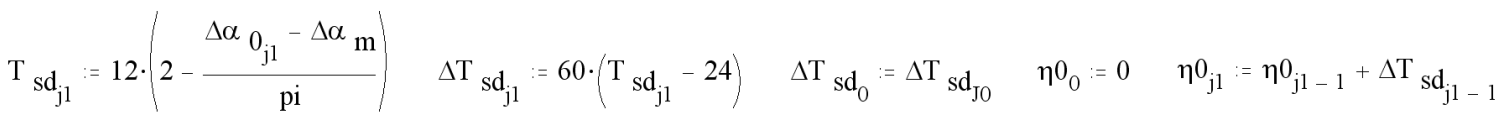

$$
\eta 0 \mathrm{~m}:=\frac{\sum_{\mathrm{j} 1=1}^{\mathrm{J} 0} \eta_{\mathrm{j} 1}}{\mathrm{~J} 0} \eta_{\mathrm{j} 1}:=\eta_{\mathrm{j} 1}-\eta 0 \mathrm{~m} \quad \mathrm{~m}_{\mathrm{j} 0}:=24 \cdot \mathrm{j} 0 \quad \mathrm{~m}_{\mathrm{a}_{\mathrm{j} 1-1}:=\mathrm{m}_{\mathrm{j} 1-1}+\frac{\eta_{\mathrm{j} 1}}{60} \quad \eta 0 \mathrm{~m}=7.800931844698135}
$$




$$
\begin{aligned}
& \operatorname{mn} \Delta \mathrm{T}:=\min \left(\Delta \mathrm{T}_{\mathrm{Sd}}\right) \quad \operatorname{Ind}_{\mathrm{j} 1}:=\text { if }\left\langle\Delta \mathrm{T}_{\mathrm{Sd}_{\mathrm{j} 1}}>\mathrm{mn} \Delta \mathrm{T}, 0, \mathrm{j} 1\right) \quad \text { in } 1:=\max (\operatorname{Ind} 1) \quad \text { in } 1=2.78 \cdot 10^{2} \quad \operatorname{mn} \Delta \mathrm{T}=-0.498094073903133 \\
& \operatorname{mx} \Delta \mathrm{T}:=\max \left(\Delta \mathrm{T}_{\mathrm{Sd}}\right) \quad \operatorname{Ind} 2_{\mathrm{j} 1}:=\mathrm{if}\left(\Delta \mathrm{T}_{\mathrm{Sd}}<\operatorname{mx} \Delta \mathrm{T}, 0, \mathrm{j} 1\right) \quad \text { in } 2:=\max (\operatorname{Ind} 2) \quad \text { in } 2=1.81 \cdot 10^{2} \quad \operatorname{mx} \Delta \mathrm{T}=0.356863208870237 \\
& \operatorname{mn} \eta:=\min (\eta) \quad \operatorname{Ind} 3_{j 1}:=\text { if }\left(\eta_{\mathrm{j} 1}>\operatorname{mn} \eta, 0, j 1\right) \quad \text { in } 3:=\max (\operatorname{Ind} 3) \quad \text { in } 3=3.29 \cdot 10^{2} \quad \operatorname{mn} \eta=-14.24894101461718 \\
& \operatorname{mx} \eta:=\max (\eta) \quad \operatorname{Ind}_{\mathrm{j} 1}:=\text { if }\left(\eta_{\mathrm{j} 1}<\operatorname{mx\eta }, 0, \mathrm{j} 1\right) \quad \text { in } 4:=\max (\operatorname{Ind} 4) \quad \text { in } 4=2.29 \cdot 10^{2} \quad \operatorname{mx} \eta=16.43486675132898
\end{aligned}
$$$$
\mathrm{P} 1_{\mathrm{i} 4,0}:=0.1 \cdot \mathrm{T} 2_{\mathrm{i} 4} \mathrm{P} 1_{0,1}:=7.800931844698135 \mathrm{P} 1_{1,1}:=7.216925795238013 \quad \mathrm{P} 1_{2,1}:=-2.545816989581148
$$$$
\mathrm{Pl}_{3,1}:=-4.696241148416599 \quad \mathrm{P}_{4,1}:=6.450777444370113 \quad \mathrm{P} 1_{0,2}:=16.43486675132898 \quad \mathrm{P} 1_{1,2}:=12.10475850450183
$$$$
P 1_{2,2}:=11.8226541833228 \quad P 1_{3,2}:=26.1632006562291 \quad P 1_{4,2}:=9.007463156468196 \quad P 1_{0,3}:=-14.24894101461718
$$$$
P 1_{1,3}:=-18.07000232096482 \quad P 1_{2,3}:=-14.735995795436 \quad P 1_{3,3}:=-19.22857210626444 \quad P 1_{4,3}:=-8.543187424642307
$$$$
\mathrm{Pl}_{0,4}:=1.720303526244837 \cdot 10^{-2} \mathrm{Pl}_{1,4}:=1.720336417940061 \cdot 10^{-2} \quad \mathrm{P}_{2,4}:=1.720235956788101 \cdot 10^{-2}
$$$$
\mathrm{P1}_{4,4}:=1.720250036785764 \cdot 10^{-2} \text { WwwwRITEPRN("TimeEq.prn") }:=\mathrm{P} 1 \quad \quad \mathrm{P} 1_{3,4}:=1.720418344610848 \cdot 10^{-2}
$$
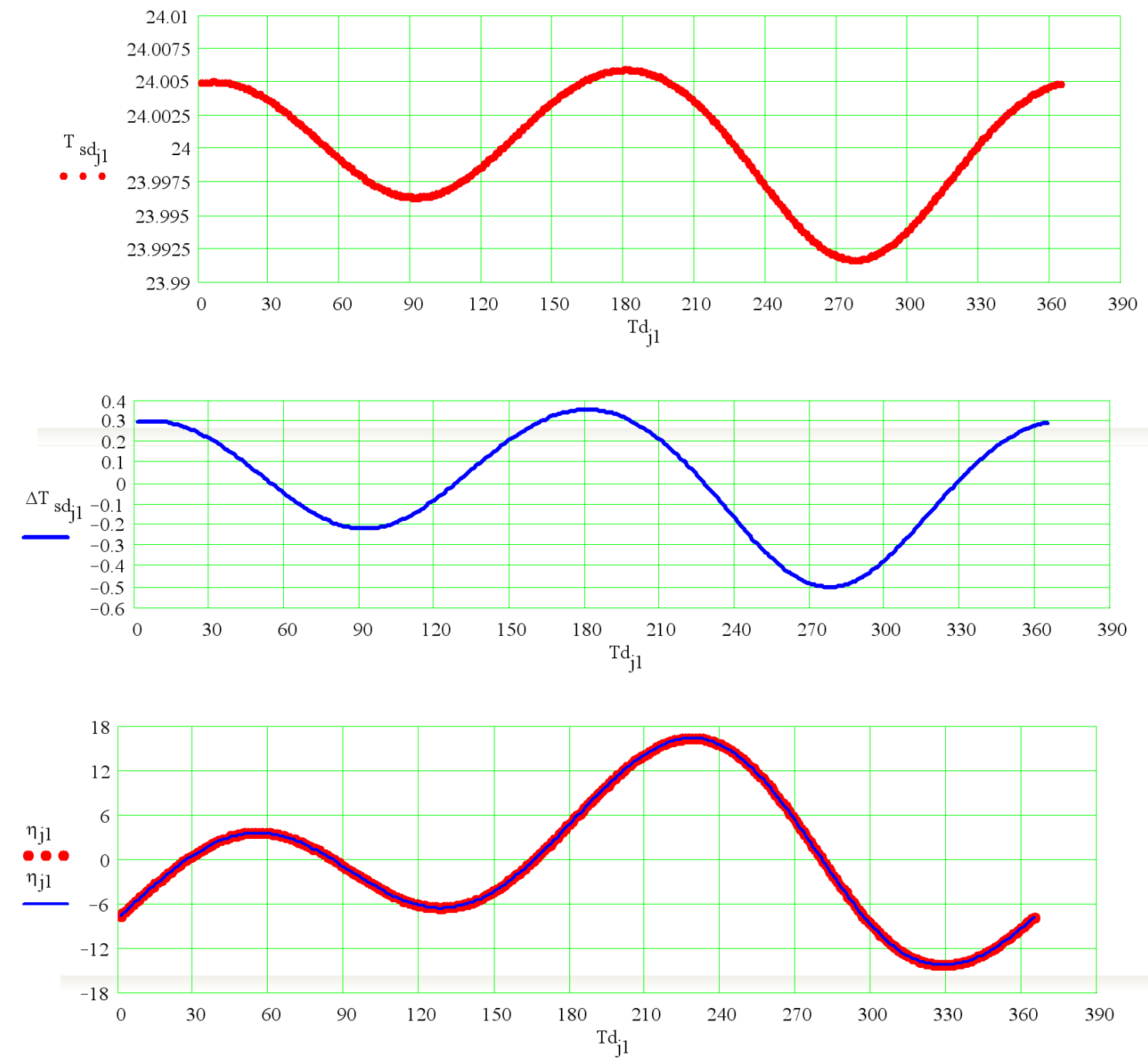


\section{Sun day length during a year (diurnal hours)}

Setting the number of the day $\mathrm{j} 11$ from the moment of $\begin{array}{llll}93 & 186 & 276 & 81\end{array}$ $\mathrm{j} 11:=81 \quad$ il $1=36 \quad \phi \mathrm{g}_{\mathrm{i} 11}=70.3622$ equinox.

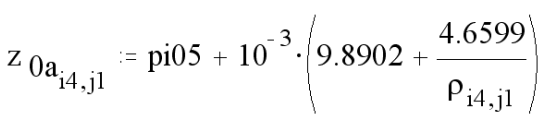

$$
\begin{aligned}
& \operatorname{Fn}_{i 4, j 1}:=\left(\frac{\cos \left(\mathrm{z}_{0 a_{i 4, j 1}}\right)-\sin \left(\delta_{i 4, j 1}\right) \cdot \sin \left(\phi_{i 11}\right)}{\cos \left(\delta_{i 4, j 1}\right) \cdot \cos \left(\phi_{i 11}\right)}\right) \\
& \mathrm{Fn}_{\mathrm{i} 4, \mathrm{j} 1}:=\text { if }\left(-1<\mathrm{Fn} 0_{\mathrm{i} 4, \mathrm{j} 1}, \mathrm{Fn} 0_{\mathrm{i} 4, \mathrm{j} 1},-1\right) \quad F \mathrm{~F}_{\mathrm{i} 4, \mathrm{j} 1}:=\text { if }\left(1>\mathrm{Fn}_{\mathrm{i} 4, \mathrm{j} 1}, \mathrm{Fn}_{\mathrm{i} 4, \mathrm{j} 1}, 1\right) \quad \omega 0 \mathrm{a}_{\mathrm{i} 4, \mathrm{j} 1}:=\operatorname{acos}\left\langle\mathrm{Fn}_{\mathrm{i} 4, \mathrm{j} 1}\right) \\
& \mathrm{D}_{\mathrm{i} 4, \mathrm{j} 1}:=\frac{24}{\mathrm{pi}} \cdot \omega 0 \mathrm{a}_{\mathrm{i} 4, \mathrm{j} 1} \quad \mathrm{D}_{0_{\mathrm{i} 4, \mathrm{j} 1}}:=\frac{24}{\mathrm{pi}} \cdot \omega 0_{\mathrm{i} 4, \mathrm{j} 1} \quad \mathrm{D}_{12}(\mathrm{~T} 1):=12
\end{aligned}
$$

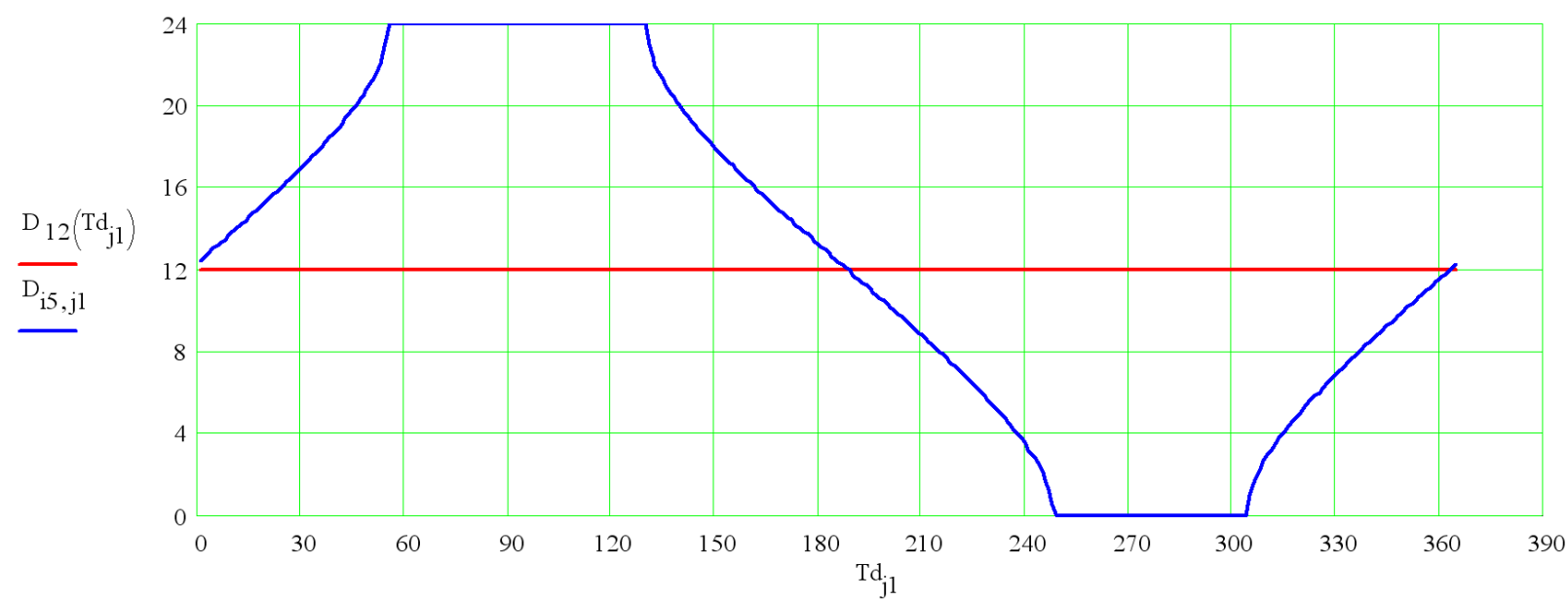

11. Polar day and polar night for Northern hemisphere

Longitude $\lambda$ of the polar day and polar night beginning.

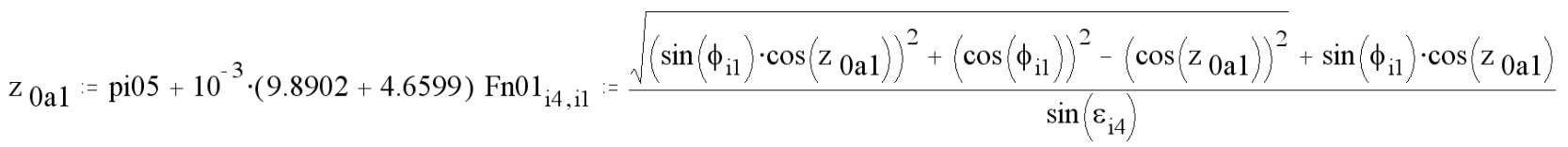

$$
\begin{aligned}
& \mathrm{Fn}_{\mathrm{i} 4, \mathrm{i} 1}=\text { if }\left(\mathrm{Fn} 01_{\mathrm{i} 4, \mathrm{i} 1} \leq 1, \mathrm{Fn} 01_{\mathrm{i} 4, \mathrm{i} 1}, 1\right) \quad \mathrm{Fn} 02_{\mathrm{i} 4, \mathrm{il}}=\frac{-\sqrt{\left(\sin \left(\phi_{\mathrm{i} 1}\right) \cdot \cos \left(\mathrm{z}_{0 \mathrm{a} 1}\right)\right)^{2}+\left(\cos \left(\phi_{\mathrm{i} 1}\right)\right)^{2}-\left(\cos \left(\mathrm{z}_{0 \mathrm{a} 1}\right)\right)^{2}+\sin \left(\phi_{\mathrm{i} 1}\right) \cdot \cos \left(\mathrm{z}_{0 \mathrm{a} 1}\right)}}{\sin \left(\varepsilon_{\mathrm{i} 4}\right)} \\
& \mathrm{Fn} 2_{\mathrm{i} 4, \mathrm{i} 1}=\mathrm{if}\left(\mathrm{Fn} 02_{\mathrm{i} 4, \mathrm{il}} \geq-1, \mathrm{Fn} 02_{\mathrm{i} 4, \mathrm{i} 1},-1\right) \\
& \lambda_{\mathrm{pd}_{\mathrm{i} 4, \mathrm{i} 1}}:=\operatorname{asin}\left(\mathrm{Fn} 1_{\mathrm{i} 4, \mathrm{il}}\right) \quad \lambda \mathrm{pd}_{\mathrm{i} 4, \mathrm{i} 1}:=\mathrm{pi}-\operatorname{asin}\left(\mathrm{Fn} 1_{\mathrm{i} 4, \mathrm{i} 1}\right) \quad \lambda_{\mathrm{pn}_{\mathrm{i} 4, \mathrm{i} 1}}=\mathrm{p} 2^{2}+\operatorname{asin}\left(\mathrm{Fn} 2_{\mathrm{i} 4, \mathrm{il}}\right) \quad \lambda \mathrm{pn} 1_{\mathrm{i} 4, \mathrm{il}}:=\mathrm{pi}-\operatorname{asin}\left(\mathrm{Fn} 2_{\mathrm{i} 4, \mathrm{i} 1}\right)
\end{aligned}
$$




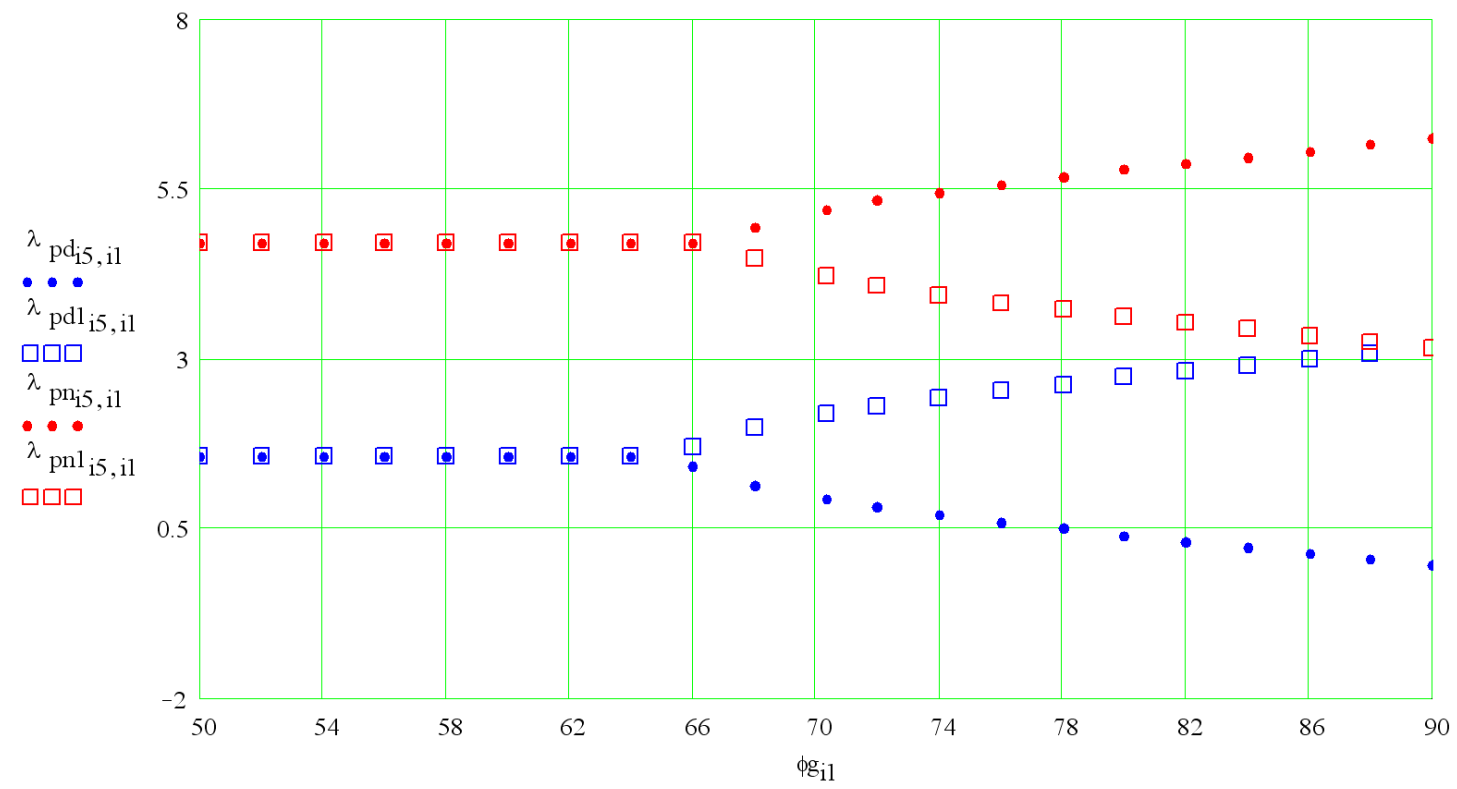

Index of of the polar day beginning

Index of of the polar day ending

$$
\begin{aligned}
& \operatorname{Ind}_{\mathrm{i} 1, \mathrm{j} 1}:=\mathrm{if}\left(\lambda \mathrm{pd}_{\mathrm{i} 5, \mathrm{i} 1} \leq \lambda_{\mathrm{i} 5, \mathrm{j} 1}, 0, \mathrm{j} 1\right) \\
& \text { in } 5:=\text { for } \mathrm{i} 1 \in 0 . . \text { If }+1
\end{aligned}
$$

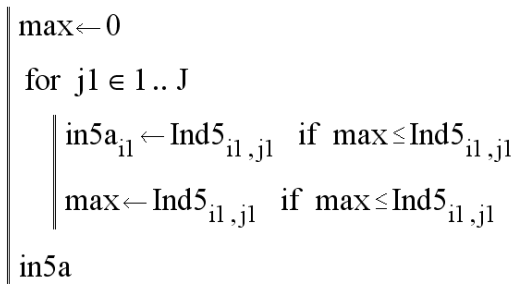$$
\operatorname{Ind}_{\mathrm{il}, \mathrm{j} 1}:=\text { if }\left(\lambda \operatorname{pd}_{\mathrm{i} 5, \mathrm{i} 1} \leq \lambda_{\mathrm{i} 5, \mathrm{j} 1}, 0, \mathrm{j} 1\right)
$$$$
\text { in } 6:=\text { for } i 1 \in 0 . . \text { If }+1
$$$$
\left\{\begin{array}{l}
\max \leftarrow 0 \\
\text { for }_{\mathrm{j} 1 \in 1 \ldots \mathrm{J}} \\
\quad \begin{array}{l}
\operatorname{in}_{\mathrm{i} 1} \leftarrow \operatorname{Ind} 6_{i 1, j 1} \text { if } \max \leq \operatorname{Ind}_{\mathrm{i} 1, \mathrm{j} 1} \\
\max _{\ln } \leftarrow \operatorname{Ind} 6_{i 1, j 1} \text { if } \max \leq \operatorname{Ind} 6_{i 1, j 1}
\end{array} \\
\text { in6a }
\end{array}\right.
$$

$\operatorname{Td}_{d_{11}}:=\operatorname{Td}_{\mathrm{in} 5_{\mathrm{i} 1}}+\frac{\left(\lambda \mathrm{pd}_{\mathrm{i} 5, \mathrm{i} 1}-\lambda_{\left.\mathrm{i} 5, \mathrm{in} 5_{\mathrm{il}}\right)}\right)\left(\mathrm{Td}_{\mathrm{in} 5_{\mathrm{i} 1}+1}-\mathrm{Td}_{\mathrm{in} 5_{\mathrm{i} 1}}\right)}{\lambda_{\mathrm{i} 5, \mathrm{in} 5_{\mathrm{i} 1}+1}-\lambda_{\mathrm{i} 5, \mathrm{in} 5_{\mathrm{i} 1}}}$

Index of of the polar night beginning

$$
\begin{aligned}
& \text { Ind } 7_{\mathrm{i} 1, \mathrm{j} 1}:=\text { if }\left(\lambda \mathrm{pn}_{\mathrm{i} 5, \mathrm{il}} \leq \lambda_{\mathrm{i}, \mathrm{j} 1}, 0, \mathrm{j} 1\right) \\
& \text { in7 }:=\text { for } \mathrm{i} 1 \in 0 . . \text { If }+1 \\
& \qquad \begin{array}{l}
\max \leftarrow 0 \\
\text { for } \mathrm{j} 1 \in 1 \ldots \mathrm{J} \\
\begin{array}{l}
\text { in } 7 \mathrm{a}_{\mathrm{i} 1} \leftarrow \operatorname{Ind} 7_{\mathrm{il}, \mathrm{j} 1} \text { if } \max \leq \operatorname{Ind} 7_{\mathrm{i} 1, \mathrm{j} 1} \\
\max _{\max } \leftarrow \operatorname{Ind} 7_{\mathrm{i} 1, \mathrm{j} 1} \text { if } \max \leq \operatorname{Ind} 7_{\mathrm{i} 1, \mathrm{j} 1}
\end{array}
\end{array}
\end{aligned}
$$

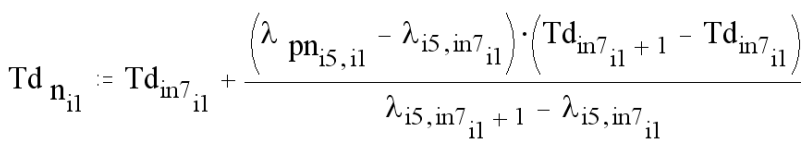

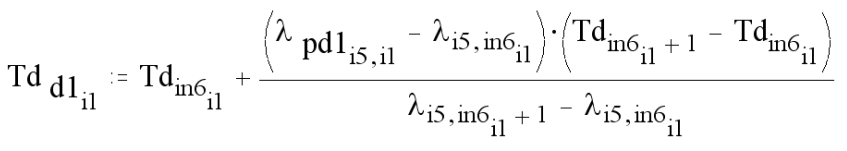

\section{Index of of the polar night ending}

$$
\begin{aligned}
& \text { Ind } 8_{\mathrm{il}, \mathrm{j} 1}:=\mathrm{if}\left(\lambda_{\mathrm{pn}} \mathrm{i}_{\mathrm{i}, \mathrm{i} 1} \leq \lambda_{\mathrm{i}, \mathrm{jl}}, 0, \mathrm{j} 1\right) \\
& \text { in } 8:=\text { for } i 1 \in 0 . . \text { If }+1 \\
& \begin{array}{l}
\max \leftarrow 0 \\
\text { for } \mathrm{j} 1 \in 1 \ldots \mathrm{J} \\
\| \begin{array}{l}
\text { in8 } \mathrm{a}_{\mathrm{il}} \leftarrow \operatorname{Ind} 8_{\mathrm{il}, \mathrm{j} 1} \quad \text { if } \max \leq \operatorname{Ind} 8_{\mathrm{il}, \mathrm{j} 1} \\
\max \leftarrow \operatorname{Ind} 8_{i 1, j 1} \text { if } \max \leq \operatorname{Ind} 8_{i 1, j 1}
\end{array} \\
\text { in8a }
\end{array}
\end{aligned}
$$

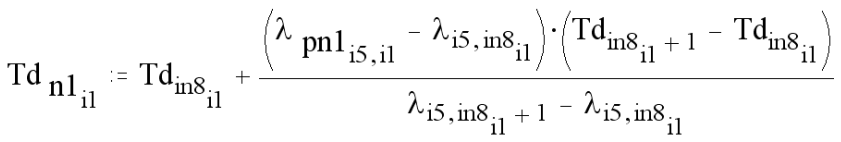

The duration of the polar days and nights. 


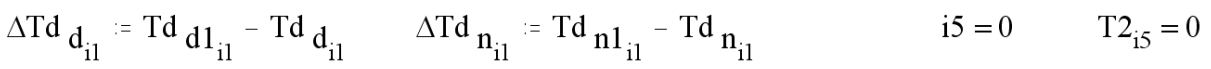

$$
\begin{aligned}
& \mathrm{P} 3_{\mathrm{i} 1,0}:=\mathrm{i} 1 \quad \mathrm{P} 3_{\mathrm{i} 1,1}:=0.1 \cdot \mathrm{T} 2_{\mathrm{i} 5} \quad \mathrm{P} 3_{\mathrm{il}, 2}:=\phi \mathrm{g}_{\mathrm{il}} \quad \mathrm{P} 3_{\mathrm{il}, 3}:=\mathrm{Td}_{\mathrm{d}_{\mathrm{i} 1}} \quad \mathrm{P} 3_{\mathrm{il}, 4}:=\Delta \mathrm{Td} \mathrm{d}_{\mathrm{i} 1} \quad \mathrm{P} 3_{\mathrm{i} 1,5}:=\mathrm{Td}_{\mathrm{n}_{\mathrm{i} 1}} \quad \mathrm{P} 3_{\mathrm{i} 1,6}:=\Delta \mathrm{Td} \mathrm{n}_{\mathrm{i} 1}
\end{aligned}
$$

WwWwRITEPRN("Tempi54.prn" ) := P3

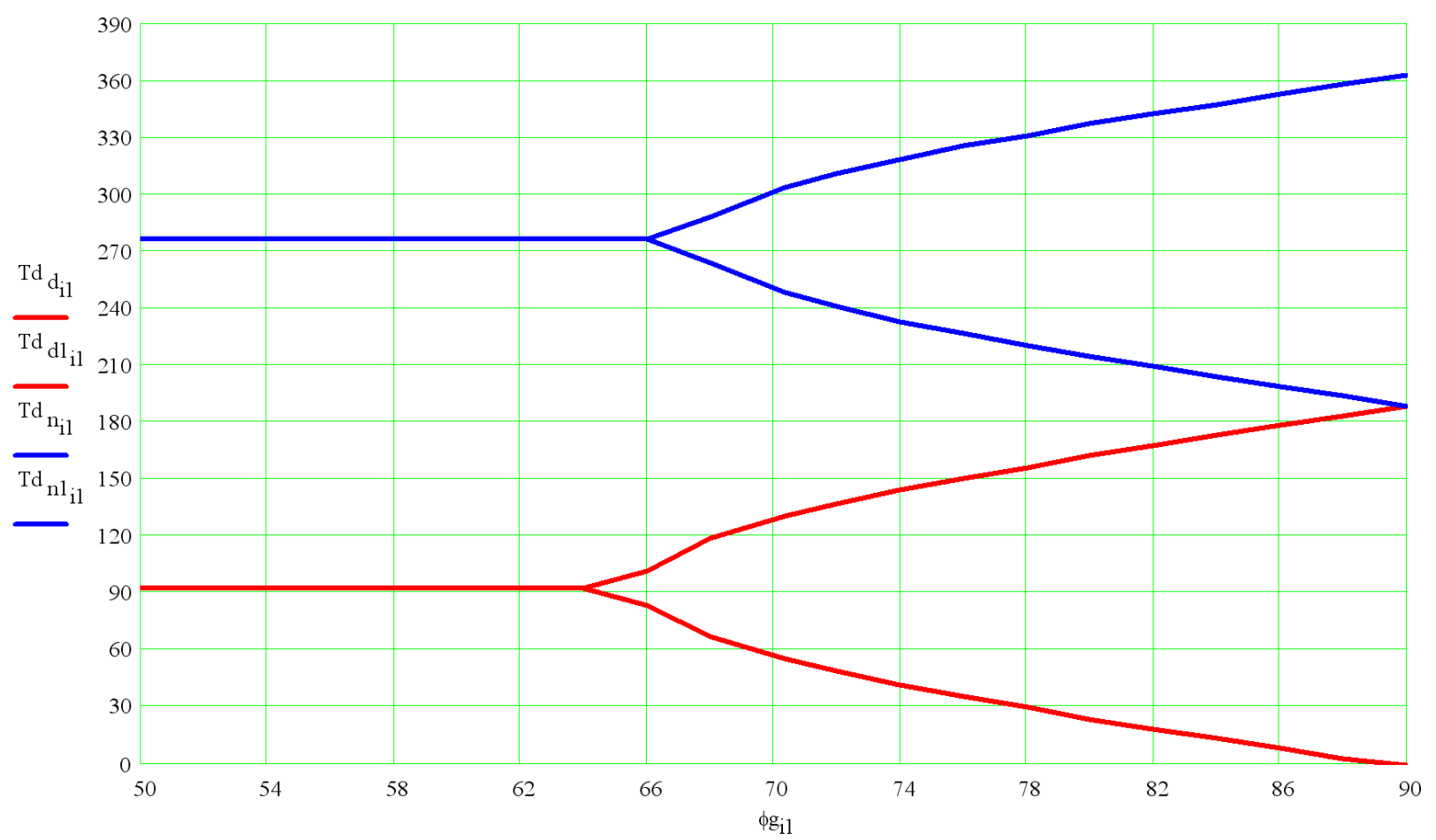

12. Extremal length of solar day at summer and winter solstices.

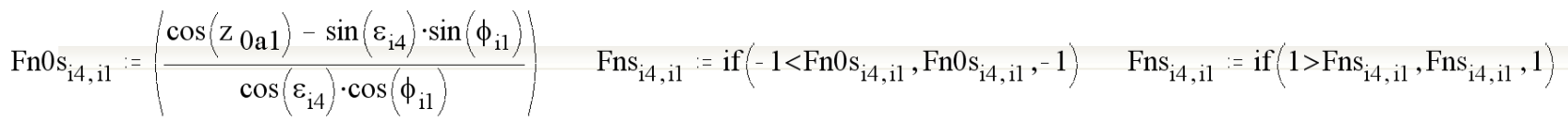
$\omega 0 \mathrm{sa}_{\mathrm{i} 4, \mathrm{il}}:=\operatorname{acos}\left(\mathrm{Fns}_{\mathrm{i} 4, \mathrm{i} 1}\right) \quad \mathrm{Ds}_{\mathrm{i} 4, \mathrm{i} 1}:=\frac{24}{\mathrm{pi}} \cdot \omega 0 \mathrm{sa}_{\mathrm{i} 4, \mathrm{il}}$

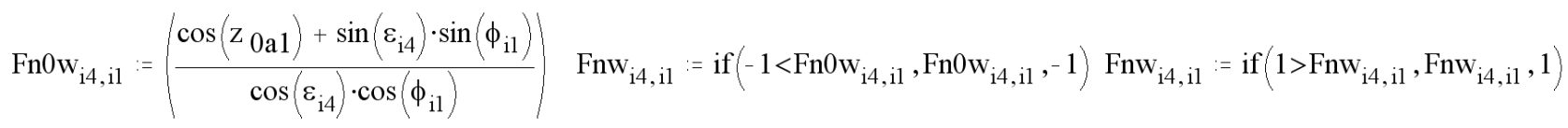
$\omega 0 \mathrm{wa}_{\mathrm{i} 4, \mathrm{il}}:=\operatorname{acos}\left(\mathrm{Fnw}_{\mathrm{i} 4, \mathrm{il}}\right) \quad \mathrm{Dw_{ \textrm {i } 4 , \mathrm { i } 1 }}:=\frac{24}{\mathrm{pi}} \cdot \omega 0 \mathrm{wa}_{\mathrm{i} 4, \mathrm{i}}$

$\mathrm{P}_{\mathrm{i} 1,0}:=0.1 \cdot \mathrm{T} 2_{\mathrm{i} 5} \quad \mathrm{P} 4_{\mathrm{i} 1,2}:=\phi \mathrm{g}_{\mathrm{i} 1} \quad \mathrm{P} 4_{\mathrm{i} 1,3}:=\mathrm{Ds}_{\mathrm{i}, \mathrm{i} 1} \quad \mathrm{P}_{\mathrm{i} 1,4}:=\mathrm{Dw}_{\mathrm{i}, \mathrm{i} 1} \quad$ WwwwRITEPRN("Tmpi54.prn") $:=\mathrm{P} 4 \quad \mathrm{i12}:=33$ 


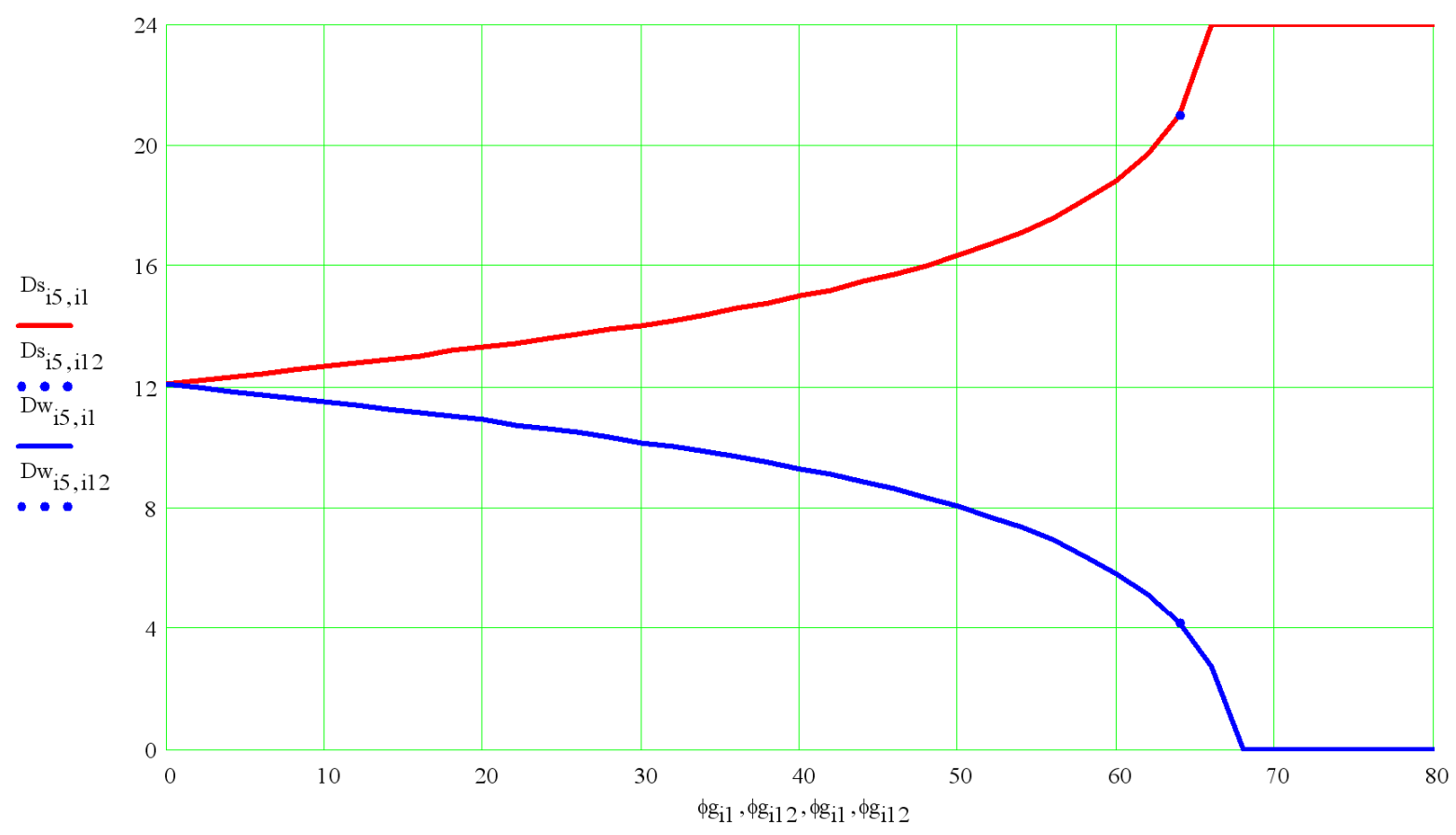

13. Azimuths of the daily movement of the Sun for the i5 epoch and j11 day of the year.

$\mathrm{K}_{\mathrm{h}}:=960 \quad \mathrm{k}:=0 . . \mathrm{K}_{\mathrm{h}} \quad \Delta \omega_{\mathrm{h}}:=\frac{24}{\mathrm{~K}_{\mathrm{h}}} \quad \omega_{\mathrm{h}_{\mathrm{k}}}:=-12+\Delta \omega_{\mathrm{h}} \cdot \mathrm{k} \quad \omega_{\mathrm{rd}_{\mathrm{k}}}:=\frac{15}{\mathrm{rd}} \cdot \omega \mathrm{h}_{\mathrm{k}} \omega_{\mathrm{h}_{\mathrm{h}}}=12 \quad \mathrm{j} 11=81 \quad \mathrm{i} 11=36$

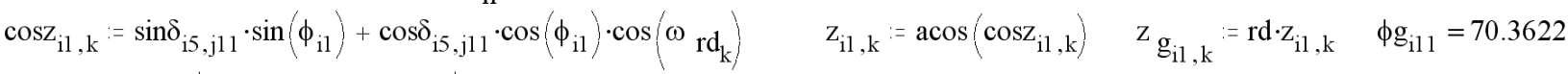

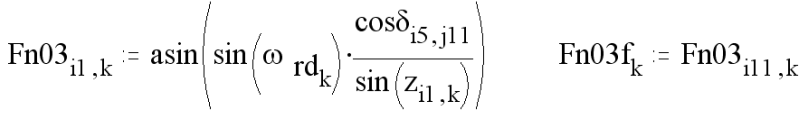

$\operatorname{mnFn} 03 f:=\min ($ Fn03f $) \quad \operatorname{Ind} 9_{k}:=$ if $\left(\right.$ Fn03 $\left.f_{k}=\operatorname{mnFn} 03 f, k, 0\right) \quad$ in $9:=\max (\operatorname{Ind} 9) \quad$ in $9=2.63 \cdot 10^{2} \operatorname{mnFn} 03 f=-1.569597608684473$ $\operatorname{mxFn} 03 \mathrm{f}:=\max (\mathrm{Fn} 03 \mathrm{f}) \quad$ Ind $10_{\mathrm{k}}:=$ if $\left(\operatorname{Fn} 03 \mathrm{f}_{\mathrm{k}}=\operatorname{mxFn} 03 \mathrm{f}, \mathrm{k}, 0\right) \quad \operatorname{in} 10:=\max (\operatorname{Ind} 10) \quad \operatorname{in} 10=6.97 \cdot 10^{2}$

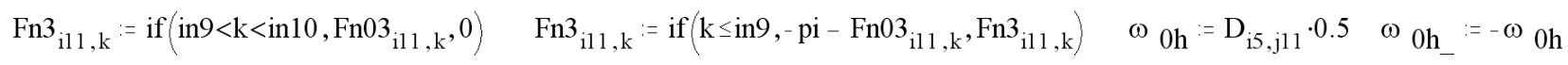

$\mathrm{Fn} 3_{\mathrm{i} 1, \mathrm{k}}:=\mathrm{if}\left(\mathrm{k} \geq \mathrm{in} 10, \mathrm{pi}-\mathrm{Fn} 03_{\mathrm{i} 11, \mathrm{k}}, \mathrm{Fn} 3_{\mathrm{i} 11, \mathrm{k}}\right) \quad \mathrm{A}_{\mathrm{S}_{\mathrm{i} 1, \mathrm{k}}}:=180+\mathrm{rd} \cdot \mathrm{Fn} 3_{\mathrm{i} 11, \mathrm{k}} \quad \mathrm{A} 0\left(\omega_{0 h}\right):=0 \quad \mathrm{~A} 0_{-}\left(\omega_{0 h}\right):=0$

Note. The minimum and maximum values Fn03 may belong to the central area. In this case, there are bursts on azimuth AS for small $\mathrm{Kh}$. In this case, it is need to change in $9<$ and $<=$ in 9 on $<$ in 10 and $>=$ in 10 :

$$
\begin{aligned}
& \left.\mathrm{Fn}_{\mathrm{il}_{1, \mathrm{k}}}:=\text { if1 11 (in9 } \leq \mathrm{k} \leq \mathrm{in} 10, \mathrm{Fn} 03_{\mathrm{i1} 1, \mathrm{k}}, 0\right) \mathrm{Fn} 3_{\mathrm{i} 11, \mathrm{k}}:=\text { if1 } 11\left(\mathrm{k}<\mathrm{in} 9,-\mathrm{pi}-\mathrm{Fn} 03_{\mathrm{i} 11, \mathrm{k}}, \mathrm{Fn} 3_{\mathrm{i} 1, \mathrm{k}}\right) \quad \operatorname{mxFn03f}=1.56959760868438 \\
& \text { Fn } 3_{i 11, \mathrm{k}}:=\text { if1 } 11\left(\mathrm{k}>\text { in10,pi }-\mathrm{Fn} 03_{\mathrm{i} 11, \mathrm{k}}, \mathrm{Fn} 3_{\mathrm{i11,k}}\right)
\end{aligned}
$$

Daily azimuth of the Sun in the tropics (In the summer at noon it is equal zero).

$$
\begin{aligned}
& \mathrm{z}_{\mathbf{n} \phi_{\mathrm{i} 1}}:=\phi_{\mathrm{i} 1}-\delta_{\mathrm{i} 5, \mathrm{j11}} \quad \mathrm{A}_{\mathrm{Stz}_{\mathrm{i} 1, \mathrm{k}}}:=180+\mathbf{r d} \cdot \mathrm{Fn} 03_{\mathrm{i} 1, \mathrm{k}} \quad \mathrm{A}_{\mathrm{St} \mathrm{z}_{\mathrm{i} 11, \mathrm{k}}}:=-\mathrm{rd} \cdot \mathrm{Fn} 03_{\mathrm{i} 11, \mathrm{k}} \\
& \mathrm{A}_{\mathrm{St}_{\mathrm{i} 1, \mathrm{k}}}:=\mathrm{if}\left(\mathrm{z}_{\mathrm{n} \phi_{\mathrm{i} 1}} \leq 0, \mathrm{~A}_{\mathrm{St} \mathrm{z}_{\mathrm{i} 11, \mathrm{k}}}, \mathrm{A}_{\mathrm{Stz}_{\mathrm{i} 11, \mathrm{k}}}\right) \quad \mathrm{A}_{\mathrm{S}_{\mathrm{i} 1, \mathrm{k}}}:=\text { if }\left(\phi_{\mathrm{i} 11} \leq \boldsymbol{\varepsilon}_{\mathrm{i} 5}, \mathrm{~A}_{\mathrm{St}_{\mathrm{i} 1, \mathrm{k}}}, \mathrm{A}_{\mathrm{S}_{\mathrm{i} 11, \mathrm{k}}}\right) \\
& \mathrm{A}_{\mathrm{M}}:=\operatorname{if}\left(\mathrm{z}_{\mathrm{n} \phi_{\mathrm{i} 11}} \leq 0,180,360\right) \quad \mathrm{A}_{\mathrm{M}_{-}}:=\text {if }\left(\mathrm{z}_{\mathrm{n} \phi_{\mathrm{i} 11} \leq 0,-180,0}\right)
\end{aligned}
$$


Daily azimuth of the Sun's center for i11 number of latitude.

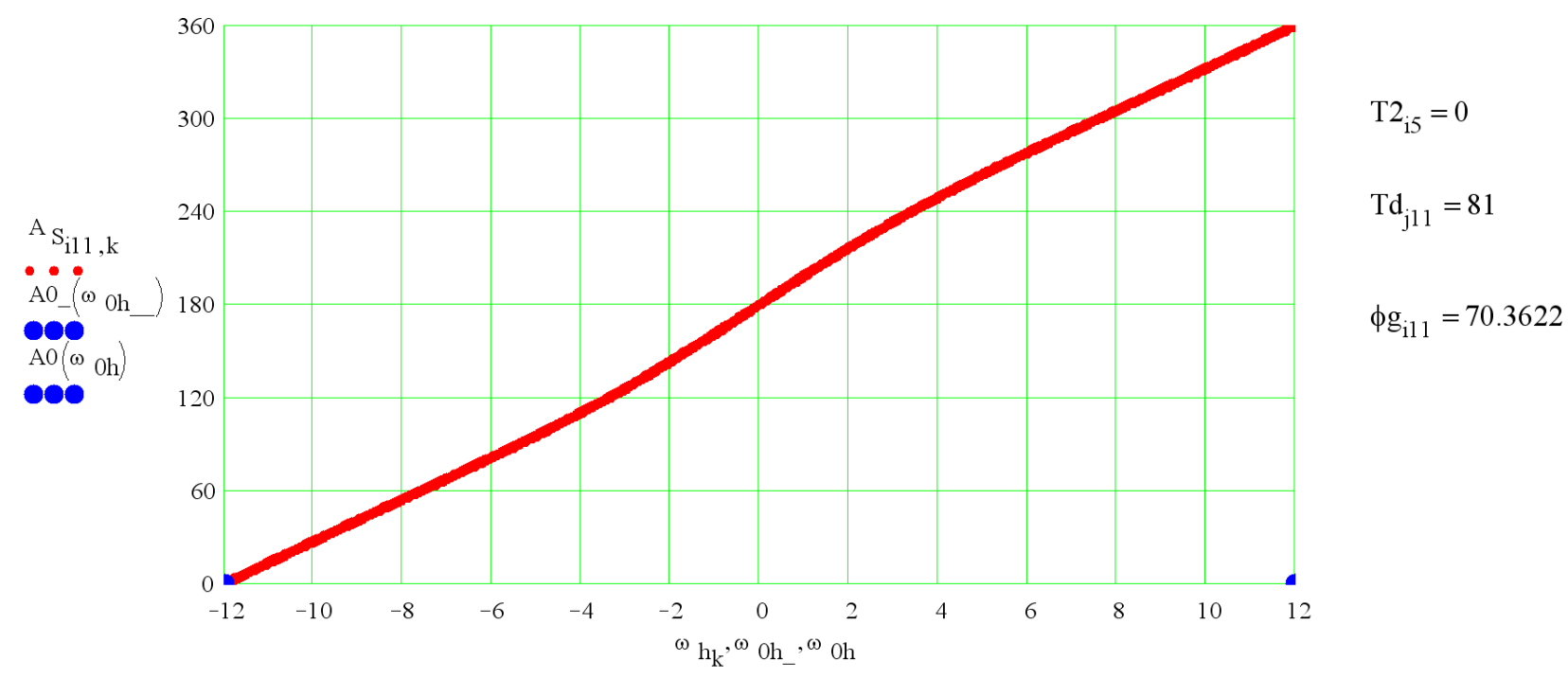

\section{Azimuths of sunrises and sunsets}

$$
\begin{aligned}
& \mathrm{Fn}_{0} 4_{\mathrm{i} 4, \mathrm{j} 1}:=\frac{\sin \left(\delta_{\mathrm{i} 4, \mathrm{j} 1}\right)}{\cos \left(\phi_{\mathrm{i} 11}\right)} \quad \mathrm{Fn} 4_{\mathrm{i} 4, \mathrm{j} 1}:=\text { if }\left(-1<\mathrm{Fn} 04_{\mathrm{i} 4, \mathrm{j} 1}, \mathrm{Fn} 04_{\mathrm{i} 4, \mathrm{j} 1},-1\right) \quad \mathrm{Fn}_{\mathrm{i} 4, \mathrm{j} 1}:=\text { if }\left(1>\mathrm{Fn}_{\mathrm{i} 4, \mathrm{j} 1}, \mathrm{Fn} 4_{\mathrm{i} 4, \mathrm{j} 1}, 1\right)
\end{aligned}
$$

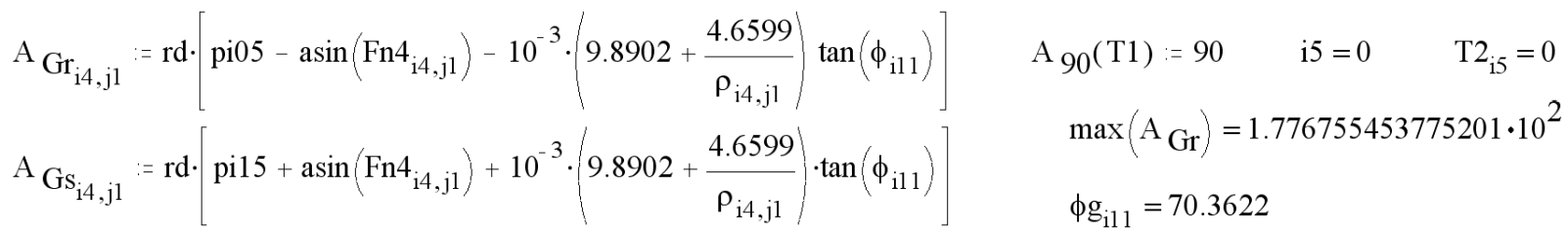

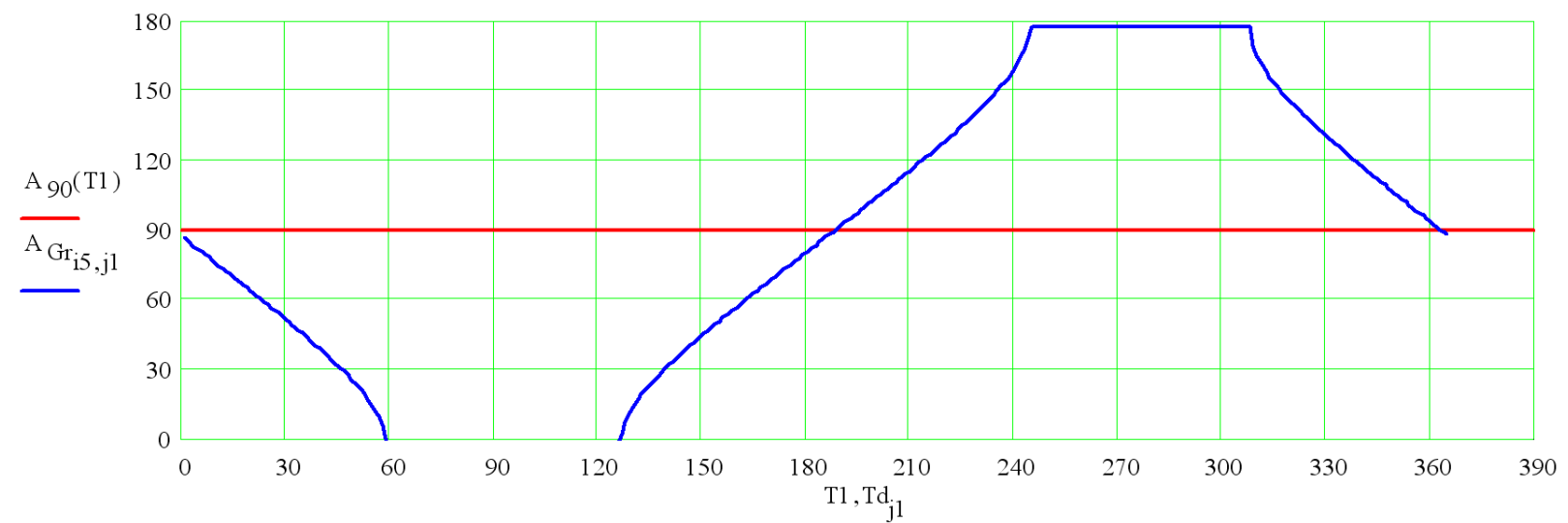

15. The projection of the daily of the Sun's path in the plane of the horizon

$$
\begin{aligned}
& \mathrm{N}_{\mathrm{S}_{\mathrm{k}}}:=\sin \left(\mathrm{z}_{\mathrm{i} 11, \mathrm{k}}\right) \cdot \cos \left(\frac{\mathrm{A}_{\mathrm{i} 11, \mathrm{k}}}{\mathrm{rd}}\right) \mathrm{E}_{\mathrm{S}_{\mathrm{k}}}=\sin \left(\mathrm{z}_{\mathrm{il1,k}}\right) \cdot \sin \left(\frac{{ }^{\mathrm{A}} \mathrm{S}_{\mathrm{i} 11, \mathrm{k}}}{\mathrm{rd}}\right) \\
& \mathrm{N}_{\mathrm{Sr}}:=\sin \left[\operatorname{pi0} 5+10^{-3} \cdot\left(9.8902+\frac{4.6599}{\rho_{\mathrm{i}, \mathrm{j} 11}}\right)\right] \cdot \cos \left(\frac{\mathrm{A}_{\mathrm{Gr}} \mathrm{i}, \mathrm{j11}}{\mathrm{rd}}\right) \mathrm{E}_{\mathrm{Sr}}:=\sin \left[\operatorname{pi05}+10^{-3} \cdot\left(9.8902+\frac{4.6599}{\rho_{\mathrm{i} 5, \mathrm{j} 11}}\right)\right] \cdot \sin \left(\frac{\mathrm{A}_{\mathrm{Gr}_{\mathrm{i}, \mathrm{j} 11}}}{\mathrm{rd}}\right)
\end{aligned}
$$


$\mathrm{N}_{\mathrm{Ss}}:=\sin \left[\mathrm{pi05}+10^{-3} \cdot\left(9.8902+\frac{4.6599}{\rho_{\mathrm{i} 5, \mathrm{j} 11}}\right)\right] \cdot \cos \left(\frac{\mathrm{A}_{\mathrm{Gs}} \mathrm{i}, \mathrm{j} 11}{\mathrm{rd}}\right) \mathrm{E}_{\mathrm{Ss}}:=\sin \left[\mathrm{pi05}+10^{-3} \cdot\left(9.8902+\frac{4.6599}{\rho_{\mathrm{i} 5, \mathrm{j} 11}}\right)\right] \cdot \sin \left(\frac{{ }^{\mathrm{A}} \mathrm{Gs}_{\mathrm{i} 5, \mathrm{j} 11}}{\mathrm{rd}}\right)$

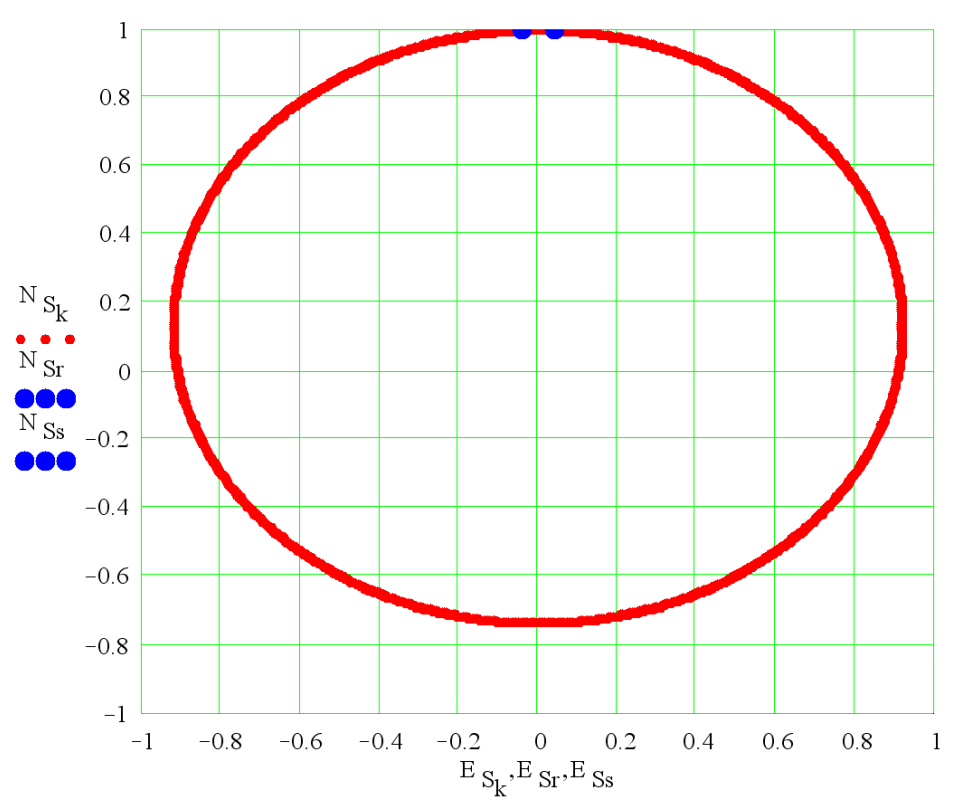

$$
\phi \mathrm{g}_{\mathrm{il} 1}=70.3622
$$$$
\mathrm{Td}_{\mathrm{j} 11}=81
$$

\section{Extreme azimuths of sunrises and sunsets during the solstices.}

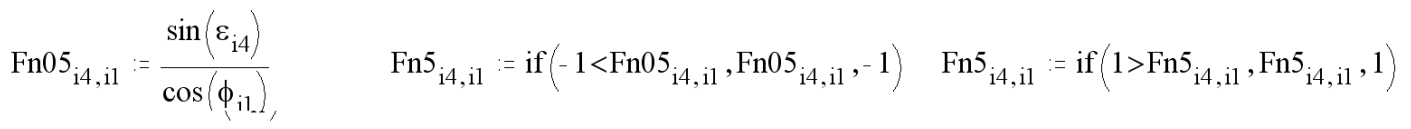

$$
\begin{aligned}
& \mathrm{A}_{\mathrm{GrS}_{\mathrm{i} 4, \mathrm{il}}}:=\mathrm{rd} \cdot\left[\mathrm{pi05}-\operatorname{asin}\left(\mathrm{Fn}_{\mathrm{i} 4, \mathrm{il}}\right)-10^{-3} \cdot\left(9.8902+\frac{4.6599}{1}\right) \cdot \tan \left(\phi_{\mathrm{il}}\right)\right] \\
& \mathrm{A}_{\mathrm{Grw}_{\mathrm{i} 4, \mathrm{i} 1}}:=\mathrm{rd} \cdot\left[\operatorname{pi0}+\operatorname{asin}\left(\mathrm{Fn}_{\mathrm{i} 4, \mathrm{i}}\right)-10^{-3} \cdot\left(9.8902+\frac{4.6599}{1}\right) \cdot \tan \left(\phi_{i 1}\right)\right]
\end{aligned}
$$$$
\mathrm{P}_{\mathrm{il}, 0}:=0.1 \cdot \mathrm{T} 2_{\mathrm{i} 5} \quad \mathrm{P} 5_{\mathrm{il}, 2}:=\phi \mathrm{g}_{\mathrm{il}} \quad \mathrm{P} 5_{\mathrm{il}, 3}:=\mathrm{A}_{\mathrm{Grs}_{\mathrm{i}, \mathrm{i}}} \quad \mathrm{P} 5_{\mathrm{i} 1,4}:=\mathrm{A}_{\mathrm{Grw}_{\mathrm{i}, \mathrm{i} 1}} \quad \text { WwwwRITEPRN("Tmi54.prn") }:=\mathrm{P} 5
$$$$
\mathrm{T}_{2}{ }_{\mathrm{i} 5}=0 \quad \mathrm{i} 12=33 \quad \phi \mathrm{g}_{\mathrm{i} 12}=64.00000000000004
$$

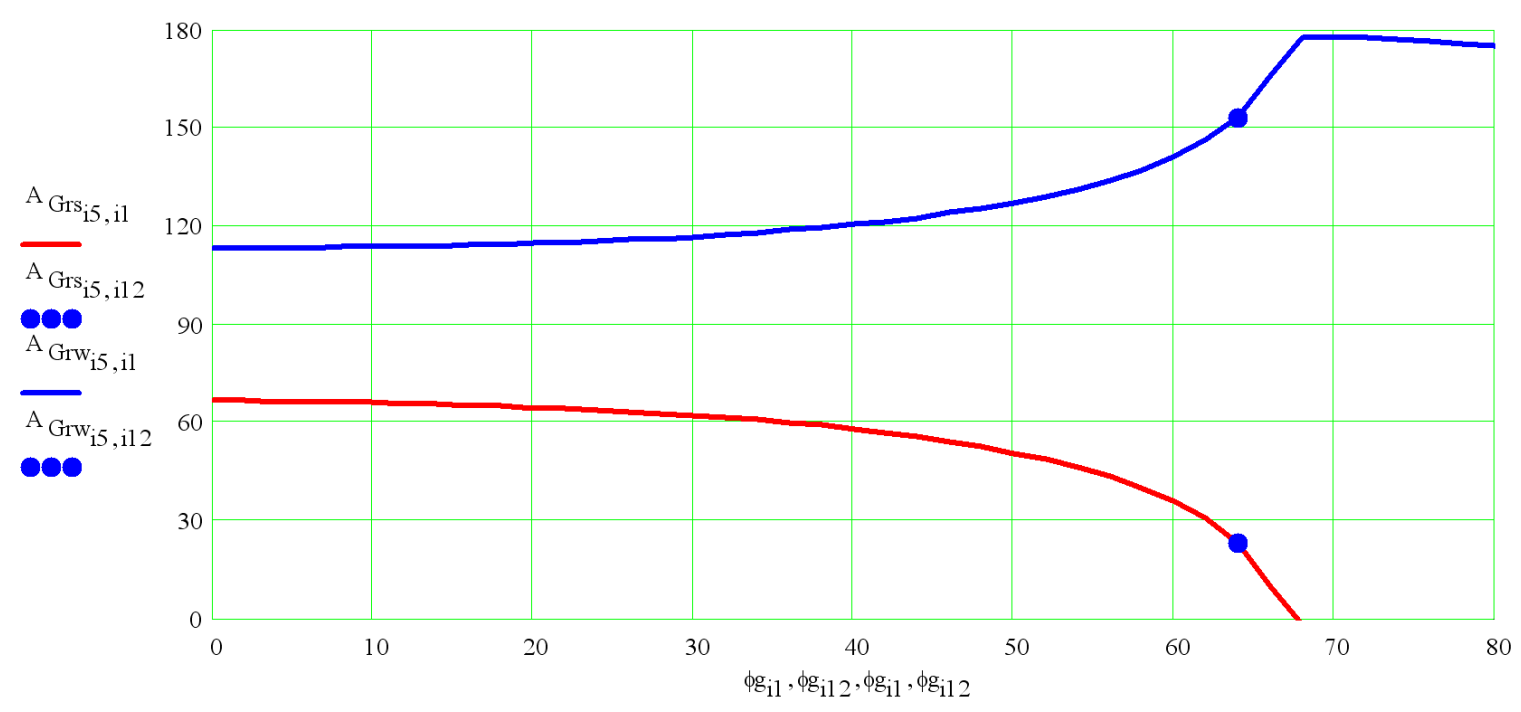




\section{Gnomon's solar shadow}

17.1. The length of the gnomon's solar shadow during the day for the $\mathrm{i} 5$ epoch, $\mathrm{j} 11$ day of the year and $\mathrm{i} 11$ latitude.

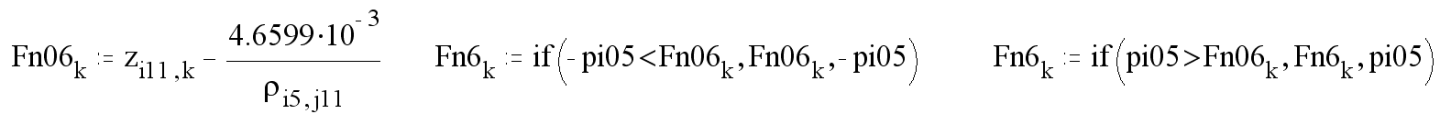

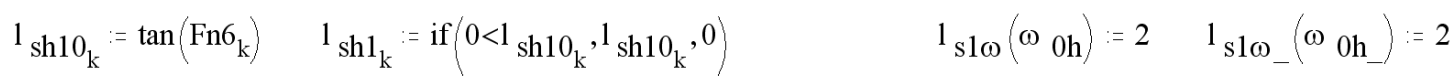

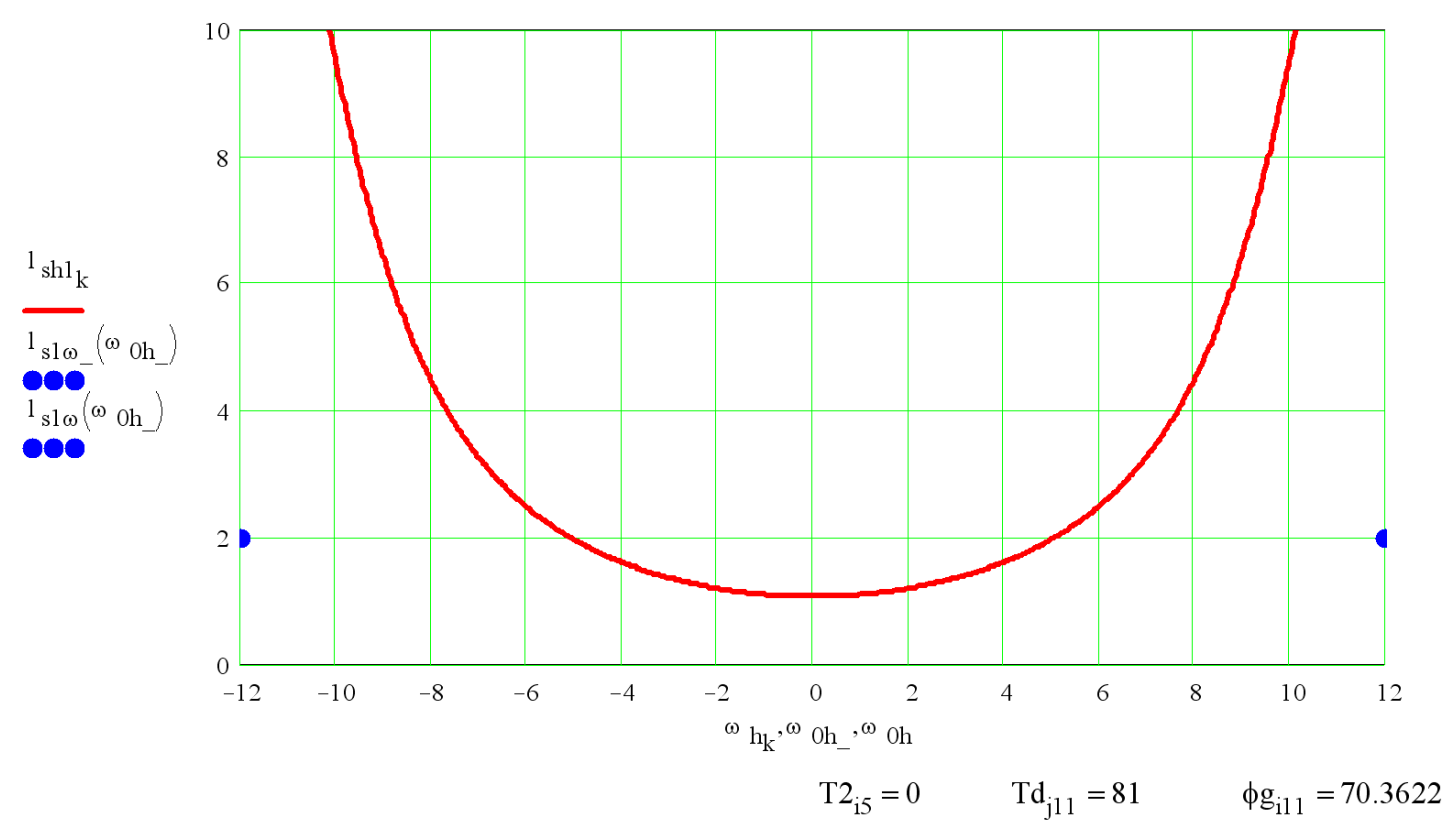

17.2. The daily path of the gnomon's shadow on the horizontal plane.

$\mathrm{N}_{\mathrm{sh}_{\mathrm{k}}}:=-1_{\mathrm{sh}_{\mathrm{k}}} \cdot \cos \left(\frac{\mathrm{A}_{\mathrm{S}_{\mathrm{i} 1, \mathrm{k}}}}{\mathrm{rd}}\right) \quad \mathrm{E}_{\mathrm{sh}_{\mathrm{k}}}=-1_{\mathrm{sh}_{\mathrm{k}}} \cdot \sin \left(\frac{\mathrm{A}_{\mathrm{i} 11, \mathrm{k}}}{\mathrm{rd}}\right)$

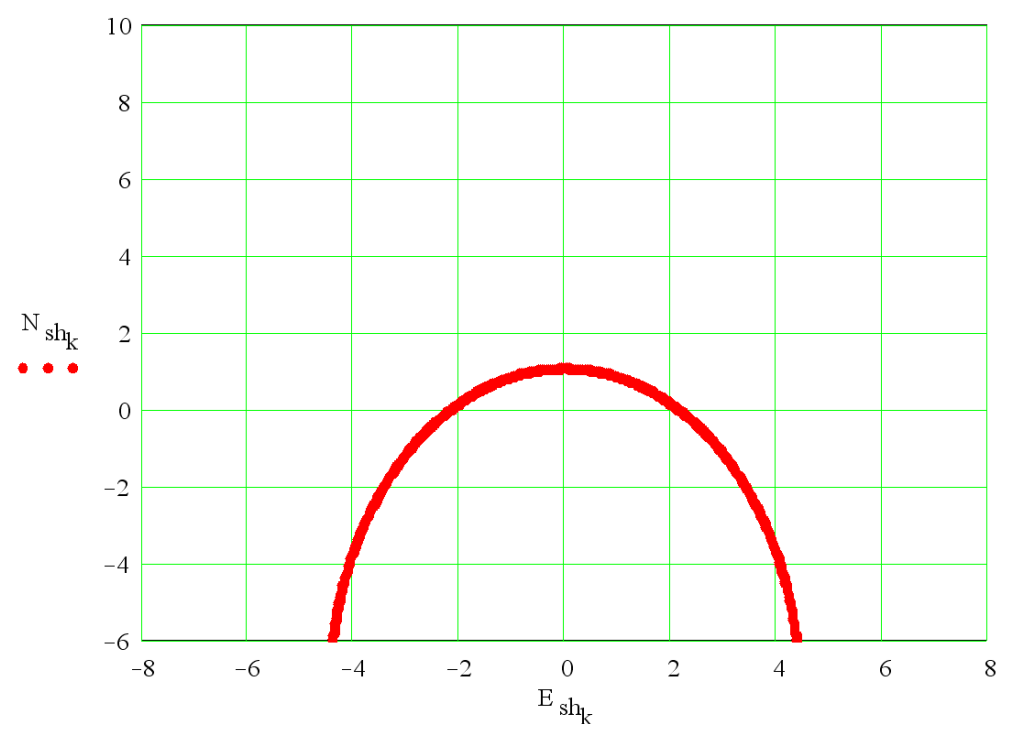

$$
\begin{aligned}
& \mathrm{T} 2_{\mathrm{i} 5}=0 \\
& \mathrm{Td}_{\mathrm{j} 11}=81 \\
& \phi \mathrm{g}_{\mathrm{i} 11}=70.3622
\end{aligned}
$$


17.3. The length of the gnomon's solar shadow in midday.

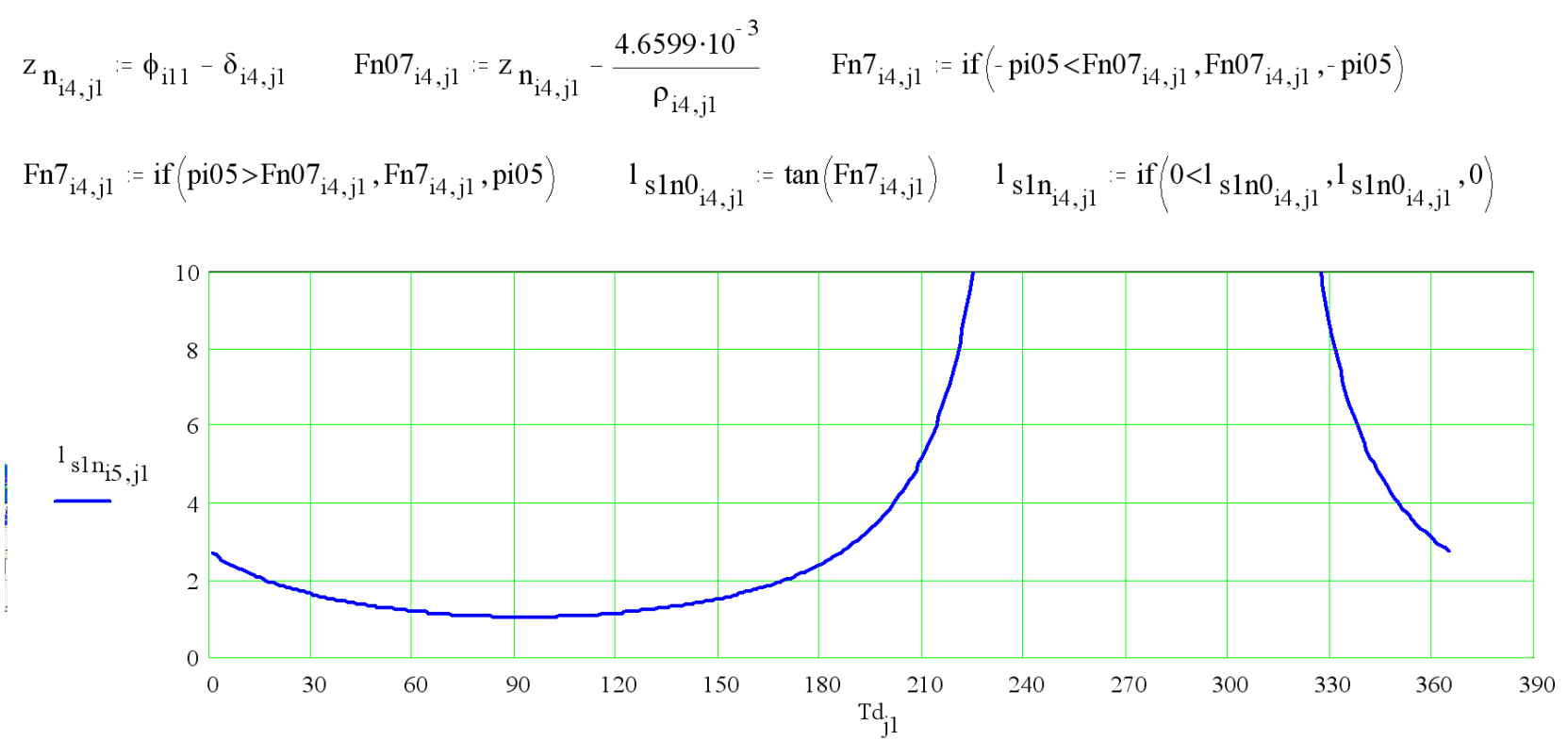

17.4. The extreme lengths of the midday gnomon's solar shadow in the solstices.

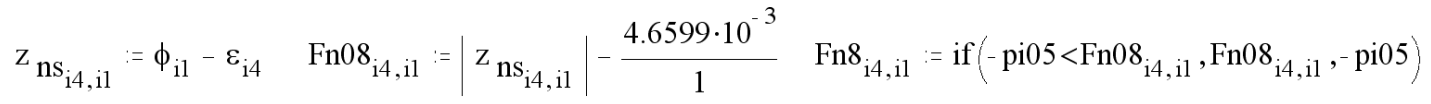

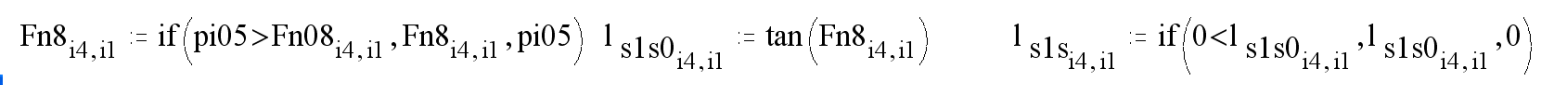

$$
\begin{aligned}
& \mathrm{z}_{\mathrm{nw}_{\mathrm{i} 4, \mathrm{i} 1}}:=\phi_{\mathrm{i} 1}+\varepsilon_{\mathrm{i} 4} \quad \mathrm{Fn} 09_{\mathrm{i} 4, \mathrm{i} 1}:=\mid \mathrm{z}_{\mathrm{nw}_{\mathrm{i} 4, \mathrm{i} 1} \mid-\frac{4.6599 \cdot 10^{-3}}{1}} \mathrm{Fn} 9_{\mathrm{i} 4, \mathrm{i} 1}:=\mathrm{if}\left(-\mathrm{pi0}<<\mathrm{Fn} 09_{\mathrm{i} 4, \mathrm{i} 1}, \mathrm{Fn} 09_{\mathrm{i} 4, \mathrm{i} 1},-\mathrm{pi} 05\right)
\end{aligned}
$$

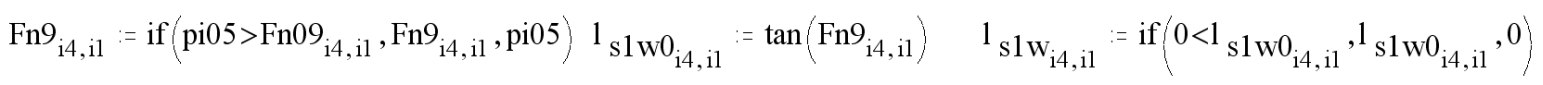

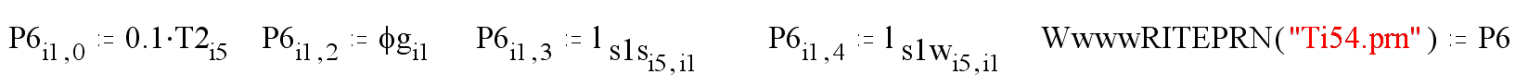

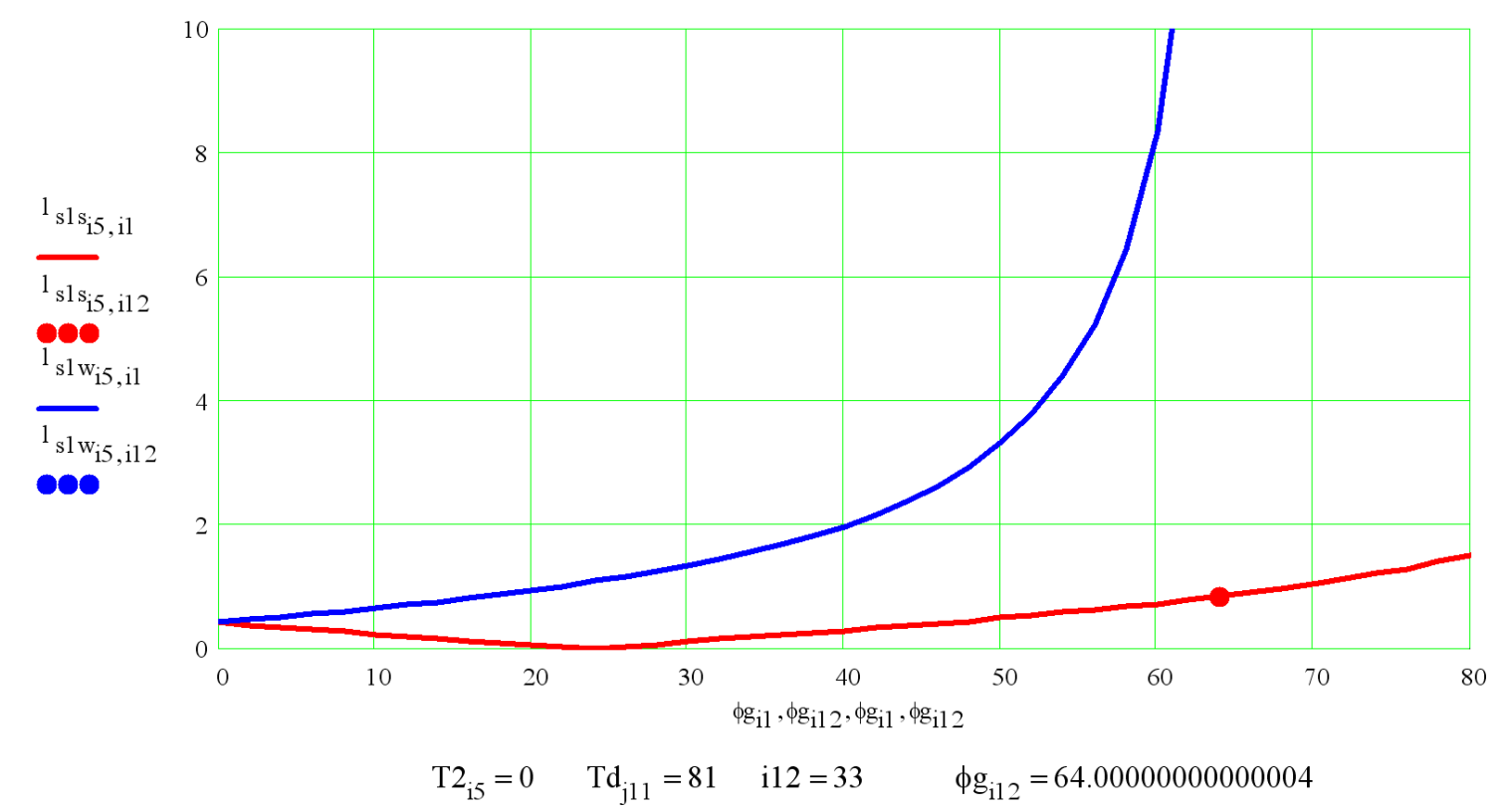


18. Using the results in the aerospace studies of relief.

The crater on the Yamal Peninsula $30 \mathrm{~km}$ south of Bovanenkovo (70.3622 degrees north latitude) is was found in the summer of 2014. On the date of 06.09 .2013 , which is remote to 81 days from the day of the vernal equinox March 21, the hillock was observed on the crater site. Azimuth of the hillock's shadow is 326.5 degrees.

$$
\phi \mathrm{g}_{\mathrm{hl}}:=70.3622 \quad \mathrm{Td}_{\mathrm{hl}}:=81 \quad \mathrm{~A}_{\mathrm{Shl}}:=326.5 \quad \mathrm{I}_{\mathrm{hl}}:=20 \quad \mathrm{i}:=0 . . \mathrm{I} \mathrm{hl}_{\mathrm{jl}} \quad \mathrm{Td}_{\mathrm{j} 11}=81 \quad \phi \mathrm{g}_{\mathrm{i} 11}=70.3622 \quad \mathrm{~T}_{\mathrm{i} 5}=0 \quad \mathrm{i} 11=36
$$

The hour angle of the Sun's azimuth at the time of occurrence of the hillock's shadow.

$$
\operatorname{Ind} 12_{\mathrm{k}}=\operatorname{if}\left(\mathrm{A}_{\mathrm{S}_{\mathrm{i} 11, \mathrm{k}}}<\mathrm{A} \operatorname{Shl}, \mathrm{k}, 0\right) \quad \text { in12 }:=\max (\operatorname{Ind} 12) \quad \operatorname{in} 12=8.62 \cdot 10^{2} \quad \mathrm{~A}_{\mathrm{S}_{111, \mathrm{in} 12}}=3.262979064470675 \cdot 10^{2}
$$

$\omega_{\mathrm{hS}}:=\omega_{\mathrm{h}_{\mathrm{in} 12}}+\frac{\left({ }^{\omega} \mathrm{h}_{\mathrm{in} 12+1}-\omega \mathrm{h}_{\mathrm{in} 12}\right) \cdot\left(\mathrm{A}_{\left.\mathrm{S}_{\mathrm{i} 1, \mathrm{in} 12+1}-\mathrm{A}_{\mathrm{Shl}}\right)}\right.}{\mathrm{A}_{\mathrm{S}_{\mathrm{i} 1, \mathrm{in} 12+1}-\mathrm{A}_{\mathrm{S} 11, \mathrm{in} 12}}} \quad \mathrm{~A}_{\mathrm{S} 1}\left(\omega_{\mathrm{h} 1}\right):=\mathrm{A}_{\mathrm{Sh} 1} \quad \omega_{\mathrm{hS}}=9.560166372856854$

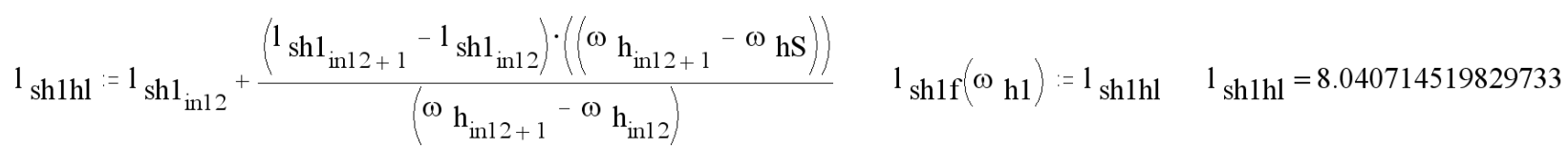

The observed radius of the hillock and length of its shadow

$\mathrm{R} 2:=$ READPRN("HillSh.prn") $\quad \mathrm{r}_{\mathrm{i}}:=\mathrm{R} 2_{0, \mathrm{i}} \quad{ }^{1} \mathrm{Osh}_{\mathrm{i}}:=\mathrm{R} 2_{1, \mathrm{i}}$

Profile of the hillock and its approximations.

$\mathrm{H}_{\mathrm{i}}:=\frac{{ }^{1} \mathrm{Oshh}_{\mathrm{i}}}{1_{\text {shlhl }}} \quad \mathrm{R}_{\mathrm{h}}:=0.5 \cdot\left(-\mathrm{r}_{0}+\mathrm{r}_{\mathrm{I}_{\mathrm{hl}}}\right) \quad{ }^{1}$ Oshmx $:=\max \left(1_{\text {Osh }}\right) \quad 1_{\text {shq }}(\mathrm{rl} 1):=1_{\text {Oshmx }} \cdot\left[1-\left(\frac{\mathrm{rl}}{\mathrm{R}_{\mathrm{h}}}\right)^{2}\right] \quad \mathrm{H}_{\mathrm{mx}}:=\max (\mathrm{H})$ $\mathrm{Hes}(\mathrm{r} 1):=\mathrm{H}_{\mathrm{mx}} \cdot \cos \left[\frac{\pi}{2} \cdot\left(\frac{\mathrm{rl}}{\mathrm{R}_{\mathrm{h}}}\right)^{2}\right] \quad 1_{\text {shcs }}(\mathrm{r} \mathrm{l}):=1_{\mathrm{Oshmx}} \cdot \cos \left[\frac{\pi}{2} \cdot\left(\frac{\mathrm{r} l}{\mathrm{R}_{\mathrm{h}}}\right)^{2}\right] \quad \mathrm{R}_{\mathrm{h}}=21.2 \quad \mathrm{H}_{\mathrm{mx}}=8.755440803971084$
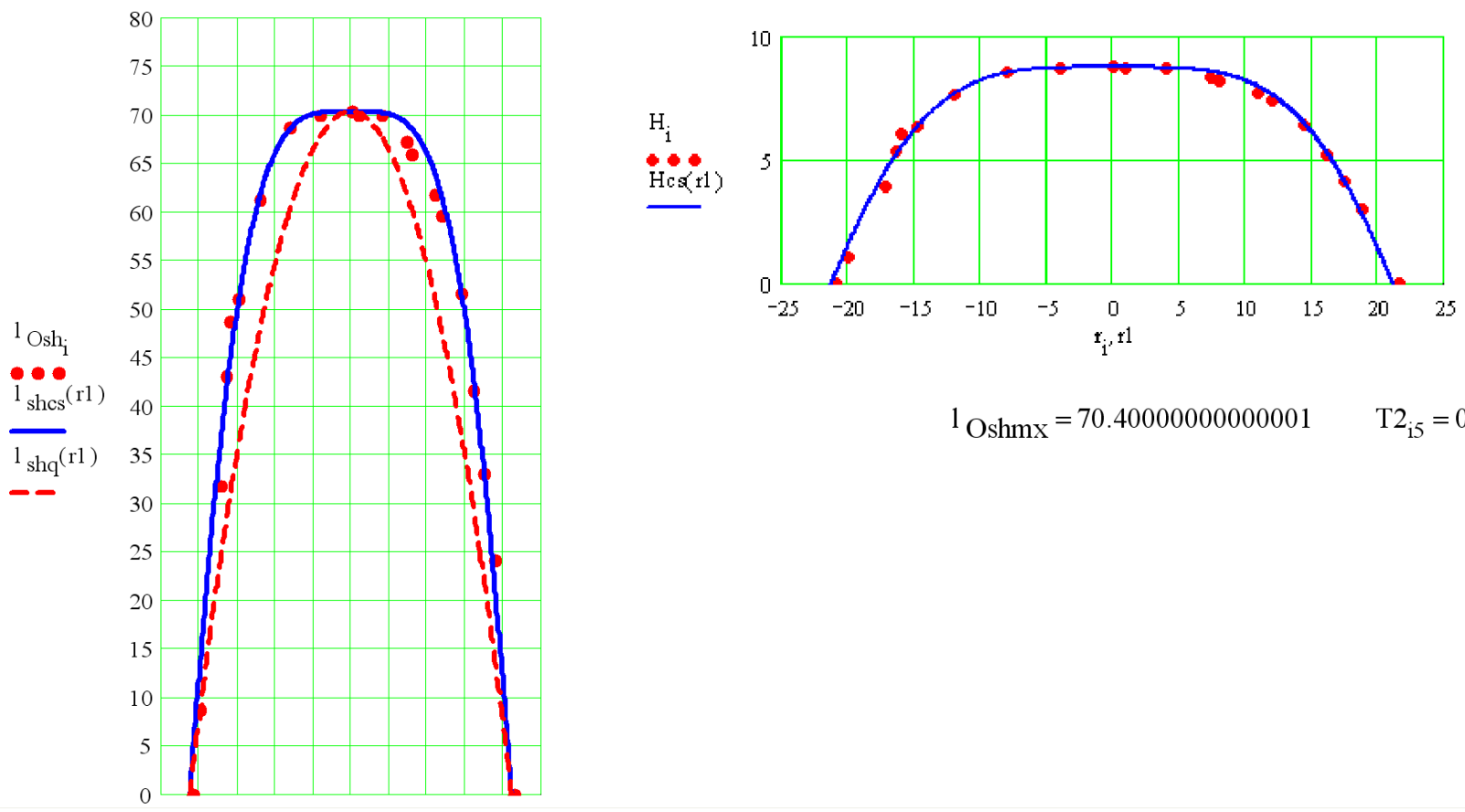

${ }^{1}$ OshmX $=70.40000000000001 \quad \mathrm{~T}_{\mathrm{i} 5}=0$ 